\title{
On the nature of isotherms at first order phase transitions for classical lattice models
}

\section{Charles-Edouard Pfister}

\begin{abstract}
Two main results are presented in this paper: Isakov's Theorem about the impossibility of an analytic continuation of the pressure at a first order phase transition point and Friedli's work about the restoration of the analytic continuation of the pressure in the van der Waals limit at such a transition point. An exposition of Pirogov-Sinai theory for models with two periodic ground-states is given in section 3, which can be read independently.
\end{abstract}





\section{Contents}

1 Introduction . . . . . . . . . . . . . . . 4

2 First order phase transition. A historical perspective . . . . 5

2.1 Natura non agit per saltum, van der Waals equation 5

2.2 First order phase transition as a singularity of the pressure ................ . . . . . 10

2.3 The van der Waals limit . . . . . . . . . . . . . 12

2.4 Lattice gas model, the Ising model . . . . . . . . . . 14

3 Phase transitions in lattice models at low temperatures . . 19

3.1 Lattice models, main assumptions . . . . . . . . . 19

3.2 Lattice models as contour models . . . . . . . . . . . 24

3.3 Construction of the phase diagram in the complex z-plane ................... 31

4 Absence of analytic continuation for lattice models with shortrange interactions . . . . . . . . . . . . . . . . . . 41

4.1 Outline of the proof of Theorem 4.1 . . . . . . . 42

4.2 Proof of Lemma 4.1 and Proposition 4.1 . . . . . . 54

4.3 Proof of Lemma $4.2 \ldots \ldots$. . . . . . . . . . . . 56

4.4 Proof of Lemma $4.4 \ldots \ldots$. . . . . . . . . 57

5 Restoration of the analyticity of the pressure at first-order phase transition in the van der Waals limit . . . . . . . . 57

5.1 Kac-Ising model. Main results . . . . . . . . . . . 59

5.2 Coarse-grained description of the model . . . . . . 62

5.3 Proof of Peierls' condition . . . . . . . . . . . 66

5.4 Polymer representation of the partition function of the restricted phases . . . . . . . . . . . 70

5.5 Properties of the restricted phases . . . . . . . . 77

$5.6 \quad$ Constrained pressure . . . . . . . . . . . . . . 81

6 Concluding remarks ................. 83

$\begin{array}{lr}\text { Bibliography } & 85\end{array}$ 


\section{Introduction}

In this paper Physics and Mathematics are interwoven. I expose two important results of Mathematical Physics about the behavior of lattice models at first order phase transitions (finite range interactions, two coexisting phases and low temperature). The first result (section 4) is Isakov's Theorem [I1], [I2], and its recent generalization [FrPf1], [FrPf3]. In 1984 Isakov proved for the Ising model that it is impossible to continue analytically the pressure in the magnetic field $h$, along the real axis, at $h=0$ and low temperature. The second one (section 5) is about the behavior of Kac-Ising models in the so-called van der Waals limit [Fr], [FrPf2]. In his PhD-thesis $[\mathrm{Fr}]$ Friedli described how analyticity of the pressure is restored, at a first order phase transition point, when the range of the interaction diverges.

In Statistical Mechanics a central problem is to determine a phase transition point. Section 2 is a summary, in a historical perspective, of two main answers given to this problem in the case of a first order transition point; one has its origin in the work of van der Waals, and the other in the work of Mayer. It is the only section written from the viewpoint of theoretical physics, and some familiarity with Statistical Mechanics may be helpful. Even if one is not familiar with Statistical Mechanics, it is important to read this section, if one wants to understand why the mathematical results, which are precisely defined and proved in the other sections, answer fundamental questions of theoretical physics, which remained open for decades. Indeed, Isakov's Theorem and its generalization (Theorem 4.1) settle, at least for lattice models, the question about the properties of the isotherms at a first order phase transition point. It confirms Mayer's viewpoint, and consequently it excludes the possibility of defining by an analytic continuation a metastable part for the isotherms at a first order phase transition. I do not touch the important topic of metastability, but refer the interested reader to the recent comprehensive book of Olivieri and Vares $[\mathrm{OV}]$, in particular chapter 4 in the context of this paper.

There is a natural way to pass from the Ising model to the mean-field Ising model, using Kac-Ising models and the van der Waals limit, which has been discovered around 1960. This allows the possibility of investigating how the analytic properties of the mean-field isotherms are restored in the van der Waals limit. Friedli was the first to consider this question in Mathematical Physics, and as far as I know even in Physics. His results underline the role of the competition between the range of the interaction and the phenomenon of phase separation in this mechanism; they give new insights on the van der Waals limit and mean-field models.

Two main topics in Mathematical Physics are treated in sections 3 and 5. Pirogov-Sinai theory $[\mathrm{PiSi}]$, which is the natural setting for formulating Isakov's Theorem, and the van der Waals limit, which is again a topic of current research. Pirogov and Sinai constructed the phase diagram of lattice models in great generality. I present the construction of phase dia- 
grams for models with two ground-states only, following the original work of Isakov [I2]. Section 3 can be read independently of the rest of the paper. When dealing with the van der Waals limit in section $5 \mathrm{I}$ expose in details the coarse-graining procedure of Bovier and Zahradník [BoZ2], which is also of general interest. This part of section 5 can also be read independently.

I learned much of the material presented here through a very fruitful and friendly collaboration with Sacha Friedli, who also made many helpful comments during the preparation of this manuscript. The invitation of Aernout van Enter and Remco van der Hofstad to lecture on this subject during the Mark Kac Seminar 2003-2004, gave me the opportunity to clarify several points with Aernout van Enter. The present paper is based, to a large part, on the unpublished lecture notes written at this occasion [Pf2].

\section{First order phase transition. A historical perspective}

\subsection{Natura non agit per saltum, van der Waals equa- tion}

In a very short period of time (1869-1875) spectacular progress has been made in our understanding of the phenomenon of phase transition for simple fluids. A dominant idea is that the gaseous and liquid states are only distant stages of the same condition of matter, and are capable of passing into one another by a process of continuous change ${ }^{1}$. This idea of continuity was not just a philosophical principle, but was clearly established experimentally by Andrews, and his results received a firm theoretical basis in the work of van der Waals. Important contributions were also made by Maxwell and Gibbs.

At equilibrium the state of a simple fluid is described e.g. by the pressure $p$, the density $\rho$ (or the specific volume $v=\rho^{-1}$ ) and the temperature $T$. These three quantities are not independent and usually one expresses the pressure as a function of $v$ and $T$. Notice that the state of the system is described by three real parameters. This means that an important assumption is implicitly made: the state of the system is supposed to be homogeneous. A basic task of a physicist is to determine which pairs $(v, T)$

\footnotetext{
${ }^{1}$ The following quotation of Herschel's Preliminary Discourse on the Study of Natural Philosophy is taken from [R] p.4. Indeed, there can be little doubt that the solid, liquid and aëriform states of bodies are merely stages in a progress of gradual transition from one extreme to the other, and that, however strongly marked the distinctions between them appear, they will ultimately turn out to be separated by no sudden or violent line of demarcation, but shade into each other by insensible gradations. The late experiments of baron Cagnard de la Tour may be regarded as a first step towards a full demonstration of this (§199). The reference to $\S 199$ of his book is to "that general law which seems to pervade all nature - the law, as it is termed, of continuity, and which is expressed in the well-known sentence "Natura non agit per saltum".
} 
correspond to gaseous states, respectively liquid states. When the temperature $T$ is fixed, an isotherm is the set of points $(p, v)$ corresponding to equilibrium states of the system at temperature $T$. Andrews' Bakerian Lecture to the Royal Society in 1869 was entitled "On the Continuity of the Gaseous and Liquid States of Matter" [An]. This paper is famous for the first experimental proof of the existence of the critical temperature, a term coined by Andrews himself in this paper. For the first time precise measurements of several isotherms for carbon dioxide were performed above, below and at the critical temperature. For a fluid above its critical temperature, Andrews observed that the ordinary gaseous and ordinary liquid states are, in short, only widely separated forms of the same condition of matter, and can be made to pass into one another by a series of gradations so gently that the passage shall nowhere present any interruption or breach of continuity. Below the critical temperature the isotherms found experimentally by Andrews are very different. It is this situation which is considered in this paper. For a temperature below the critical temperature an isotherm is qualitatively (see Figure 1) the union of the curves $A B C$ and $G H K$. The points of the curve $G H K$ of the isotherm, the vapor branch, represent gaseous equilibrium states of the system, and those of the curve $A B C$, the liquid branch, represent liquid equilibrium states. At a well-defined pressure the system can be in two distinct equilibrium states, the gaseous state $G$ and the liquid state $C$. One says that the liquid is in equilibrium with its vapor. Experimentally, the points of the horizontal curve $C G$ do not correspond to a single homogeneous equilibrium state, but their signification is as follows. At any point of the part $C G$ the system is in an inhomogeneous state, which is a mixture of liquid state $C$ and of gaseous state $G$. The only difference between two points of this horizontal segment is the proportion of liquid with respect to gas. At $G$ the whole system is in the gaseous state, and as the volume is diminished the portion of the system in the liquid state increases, so that at $C$ the whole system is in the liquid state. This is the phenomenon of condensation. There is breach of continuity, the vapor and liquid branches are distant. Today one says that there is a first order phase transition with two coexisting phases. Experimentally one can pass from one state to the other in a reversible way along the horizontal curve $C G$. Often the isotherm is defined as the whole curve $A B C G H K$ of Figure 1, which is made of the three pieces, $A B C$, the horizontal part $C G$ and $G H K$. I use this convention below.

In $1871 \mathrm{~J}$. Thomson wrote a speculative paper [Th] about the isotherms of a simple fluid ${ }^{2}$. After summarizing the experimental results of Andrews [An], proving the existence of the critical point and the fact that above

\footnotetext{
${ }^{2}$ In 1871 Maxwell was writing his book Theory of Heat and he gave an account of the works of Andrews and Thomson. Andrews' experimental isotherms are reproduced at p.120 and Thomson's ideas are discussed at p.124-127 in [M1]. The isotherms at p.125 are Thomson's speculated isotherms, not the isotherms derived by van der Waals! Van der Waals' dissertation was published in 1873.
} 


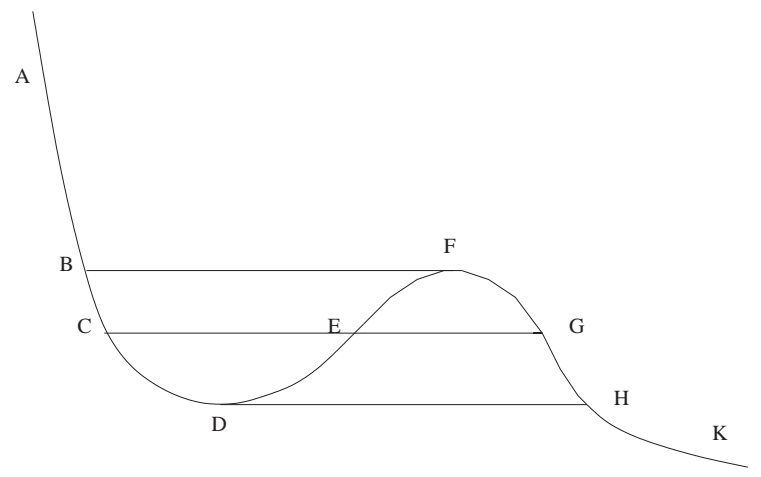

Figure 1: Thomson's isotherm below the critical temperature, pressure as function of specific volume.

the critical temperature one can pass from the gaseous state to the liquid state by a course of continuous physical changes presenting nowhere any interruption or breach of continuity, he wrote it will be my chief object in the present paper to state and support a view which has occurred to me, according to which it appears probable that, although there is a practical breach of continuity in crossing the line of boiling-points from liquid to gas or from gas to liquid, there may exist, in the nature of things, a theoretical continuity across this breach having some real and true significance. Thomson proposed the existence of an isotherm, which corresponds to the curve $A B C D E F G H K$ of Figure 1, with one local minimum at $D$ and one local maximum at $F$. According to Thomson, any point of this curve should represent a homogeneous state of matter ${ }^{3}$. The interpretation of the theoretical isotherm proposed by Thomson is essentially the one which one finds in many text-books, and which is still taught today ${ }^{4}$. The liquid branch $A B C$ and vapor branch $G H K$ have of course the same interpretation as above. The points of these branches are equilibrium states. The new points between $C$ and $D$ should represent homogeneous liquid states, which are not equilibrium, but metastable states. They represent superheated liquid states, and such states were experimentally observed in 1871 . Similarly the points between $G$ and $F$ represent supercooled vapor states which are metastable. The interpretation of the points of the isotherm be-

\footnotetext{
${ }^{3}$ Therefore the point $E$, which is also a point of the horizontal segment from $C$ to $G$, has now a different interpretation as the previous one. It should represent a homogeneous state of matter, not a mixture of a gas and a liquid.

${ }^{4}$ See for example $[\mathrm{Ca}]$ chapter 9 , or $[\mathrm{CoM}]$ chapter 8 .
} 
tween $D E F$ is much more problematic, because the pressure is increasing with the volume. This part of the isotherm is usually considered as nonphysical, following Maxwell, who did not attribute any physical meaning to this part of the isotherm (see [M1] p.125). Thomson, and later van der Waals, did not agree with Maxwell on that point. Thomson thought that the states corresponding to the unstable part of the isotherm might be realizable at the interface between gas and liquid. Moreover, these states play a crucial role in van der Waals theory of interfaces and capillarity [vdW2], as well as in Cahn-Hilliard theory (see below).

Van der Waals published his famous dissertation in 1873 [vdW1], whose title in English is almost identical to the title of Andrews' Bakerian Lecture in 1869: "On the Continuity of the Gaseous and Liquid States." It is in this work that appears the famous equation of state, which can be written

$$
\left(p+\frac{a}{v^{2}}\right)(v-b)=k T .
$$

Van der Waals derived equation (2.1) from Clausius' Virial Theorem, which relates the kinetic energy of molecules to forces acting on them $[\mathrm{Cl}]$. In that formula $a>0$ and $b$ is four times the effective volume of the molecule, so that the second factor is the effective volume, per particle, within which the molecules can move; $k$ is Boltzmann constant. Van der Waals equation gives a firm theoretical basis to Andrews' experimental results on the continuity of the gaseous and liquid states, and confirms thoroughly Thomson's speculations. There exists a critical temperature $T_{c}$ such that for $T>T_{c}$ there is only one real solution for $v$, given $p$ and $T$. On the other hand, if $T<T_{c}$ there are three real solutions, e.g. $C, E$ and $G$, and qualitatively the isotherms are similar to those of Thomson. Van der Waals' dissertation $^{5}$ received immediate recognition and Maxwell wrote a long review in Nature in 1874 [M2]. Equation (2.1) alone is not sufficient for determining the transition point where the system is in two different equilibrium states. Indeed, one does not know where are located the end-points of the liquid and vapor branches. These end-points, $C$ and $G$, are determined by a supplementary argument, Maxwell's equal area rule. Maxwell announced his argument to P.G. Tait in the following terms ${ }^{6}$ : In James Thomsons figure $^{7}$ of the continuous isothermal show that the horizontal line representing mixed liquid and vapour cuts off equal areas above 86 below the curve. Do this by Carnots cycle. That I did not do it in my book shows my invincible

\footnotetext{
${ }^{5}$ This fundamental work, its consequences and later developments are analyzed by Rowlinson in his in excellent essay [R], where an English translation of van der Waals' dissertation is also given. See also [Kl]. For a recent account of van der Waals equation see [ELi]. This reference contains the derivation of (2.1) from statistical mechanics due to Ornstein (Leiden dissertation) [Or], and which is based on the idea that the interaction pair potential between particles consists in a repulsive hard-core that is short range and an attractive, weak, long-range part.

${ }^{6} 28$ December $1874[\mathrm{Ha}]$ 155-156; see also [Ha] 157-158.

${ }^{7}$ I.e. here Figure 1.
} 
stupidity. The value $p^{*}$ of the pressure, for which there is a plateau in the isotherm, is determined by the condition

$$
p^{*}\left(v_{g}-v_{l}\right)=\int_{v_{l}}^{v_{g}} p(v) d v,
$$

$p(v)$ being the equation of the isotherm given by equation (2.1). This thermodynamic argument was published in 1875 in [M3]. There is an alternative way of determining the correct value of the pressure $p^{*}$, which is based on Gibbs' fundamental 1873 paper A Method of Geometrical Representation of the Thermodynamic Properties of Substances by Means of Surfaces [G1], where he gave a geometric characterization of the phase diagram, by introducing the convex energy-volume-entropy surface, which he called the thermodynamic surface of the body. The pressure is given (up to the sign) by the derivative of the Helmholtz free energy ${ }^{8} f$ with respect to $v$. Below the critical temperature, this free energy is not convex along the isotherm above $\left[v_{l}, v_{g}\right]$. Maxwell's rule is equivalent to Gibbs' construction: take first the convex envelope $f^{*}$ of the Helmholtz free energy $f$, and then take the derivative, so that

$$
-p^{*}=\left.\frac{\partial f^{*}}{\partial v}\right|_{v_{g}}=\left.\frac{\partial f^{*}}{\partial v}\right|_{v_{l}} .
$$

The theory of van der Waals is what is called today a mean-field type theory.

To summarize, at the end of the nineteen century, one has the following understanding of the phenomenon of condensation. The isotherms of van der Waals are analytic curves. For each fixed value of the temperature below $T_{c}$ Maxwell's rule gives the (unique) value of the pressure for which vapor and liquid coexist as equilibrium phases; the equilibrium isotherms at low temperature have three distinct analytic parts, the middle flat part defined through Maxwell's rule corresponds to physical situations where both the vapor and liquid coexist as equilibrium phases. The van der Waals isotherm gives analytic continuations for the vapor and liquid branches. Even the more problematic part of the analytic isotherm, between the local minimum at $D$ and the local maximum at $F$, where $\frac{\partial p}{\partial v}>0$, plays an essential role in the thermodynamic theory of capillarity and interfaces of van der Waals [vdW2]. This is of course also true for the more recent theory of Cahn and Hilliard [CHi], which revived and extended van der Waals' ideas (see e.g. [W1] and [W2]). This theory is still widely used today, but there are few attempts to understand, starting from the microscopic interactions, the origin of the non-convex parts of the free energies responsible for the unstable parts of the isotherms. Langer discussed in [La2] explicitly this question, and gives arguments for obtaining these free energies as

\footnotetext{
${ }^{8} f=u-T s$ gives the maximum work that can be extracted from the system along any isotherm.
} 
constrained free energies. The constraint should prevent phase separation. His arguments are however not very different from those of van Kampen [vK] (see also subsection 2.4).

\subsection{First order phase transition as a singularity of the pressure}

In 1901 Gibbs published his monograph, Elementary Principles in Statistical Mechanics, [G2]. Our understanding of phase transitions since the beginning of the 20th century, in particular the fact that we can describe with the help of a single mathematical expression, the partition function, both the liquid and the gaseous phases, is the result of many successful applications of the principles exposed in this monograph to a wide variety of physical problems. In the context considered in this paper the (canonical) partition function of a fluid of $N$ particles located at $\mathbf{x}_{i}, i=1, \ldots, N$, inside a vessel $V$, and at temperature $T$, is equal to

$$
Z(V, T, N)=\frac{1}{N ! \lambda^{3 N}} \int_{V} \cdots \int_{V} d \mathbf{x}_{1} \cdots \mathbf{x}_{N} \exp \left(-\frac{1}{k T} U\left(\mathbf{x}_{1}, \cdots, \mathbf{x}_{N}\right)\right),
$$

where $\lambda$ is an explicit $T$-dependent constant, and $U\left(\mathbf{x}_{1}, \cdots, \mathbf{x}_{N}\right)$ is the total potential energy of the $N$ particles.

In 1937 Mayer published a paper [Ma], which prompted immediately several important papers by Born [B], Born and Fuchs [BF], Kahn's dissertation (1938) at Utrecht [Ka], Kahn and Uhlenbeck [KaU]. See also De Boer [dB1] and [dB2]. A major problem in the theory of van der Waals, which has been mentioned above, is the fact that it does not provide a mechanism leading to condensation. Mayer tried to solve this problem, starting from the basic principles exposed in [G2]. If one evaluates the partition function $Z(V, T, N)$, then from the basic rules of Statistical Mechanics one gets the pressure, and in this way one can compute the isotherms of the system. Indeed, the Helmholtz free energy $f(v, T)$ is given by the limit, called thermodynamic ${ }^{9}$ limit in Statistical Mechanics,

$$
f(v, T)=-\lim _{n \rightarrow \infty} \frac{1}{k T} \frac{1}{N_{n}} \ln Z\left(V_{n}, T, N_{n}\right),
$$

where $V_{n}$ is a (suitable) sequence of vessels of volume $\left|V_{n}\right|$, so that $\left|V_{n}\right|$ and $N_{n}$ diverge as $n \rightarrow \infty$, subject to the constraint $v=\left|V_{n}\right| / N_{n}$. Then $p=$ $-\frac{\partial f}{\partial v}$. Explicit evaluation of $f(v, T)$ is in general impossible. However, one can get an expression of the pressure as a function of $T$ and a new parameter $z$ (called fugacity ${ }^{10}$ ) under the form of a convergent power series ${ }^{11}$ when $z$

\footnotetext{
${ }^{9}$ See $[\mathrm{Hu}]$ and $[\mathrm{Ru}]$, in particular $[\mathrm{Ru}] 3.4 .4$ for rigorous results. See [St] for an elementary text.

${ }^{10}$ The fugacity is defined as $z=\mathrm{e}^{\beta \mu}$, where $\mu$ is the chemical potential and $\beta=1 / k T$.

${ }^{11}$ Virial series, see e.g. [Hu]
} 
is small,

$$
\frac{p(z)}{k T}=\sum_{l \geq 1} b_{l} z^{l} .
$$

Small fugacity implies that the density of the fluid is small, which means that the system is in the gaseous phase. The problem is therefore to compute the coefficients of this series, the so-called cluster integrals $b_{l}$. Mayer assumed that the cluster integrals were independent of the volume and were positive $^{12}$ below the critical temperature. From these assumptions it follows that the analytic function $p(z)$ for small $z$ has a singularity on the positive real axis at the value of the convergence radius of the above power series. Mayer then tried to show that this singularity coincides with the value of the fugacity $z=z_{\sigma}$ at the condensation point, so that from the virial series one deduces the condensation point. Notice that this method does not allow the determination of the liquid branch of the isotherms. These ideas are best summarized by Fisher in $[\mathrm{F}]$ as Mayer's conjecture: $p(z)$, defined by its power series and its analytic continuation, has, on the positive real axis, a smallest singularity $z=z_{1}$ which occurs at $z=z_{\sigma}$, the fugacity at the condensation point. An important consequence of this conjecture, is that it is impossible to obtain metastable states, like supersaturated vapor states, by analytic continuation of the pressure along the real axis.

The mathematical deduction of the existence of a phase transition and of its properties, from the study of the partition function only, is very difficult for realistic models of physical systems. In order to avoid considering the full partition function, several authors introduced just after Mayer's paper a simpler model of condensation, which leads qualitatively to results comparable with those of Mayer's theory, the so-called droplet model ${ }^{13}$. See Bijl [Bij] (Leiden dissertation), Band [Ban], Frenkel [Fre1], [Fre2] and Mayer and Streeter $[\mathrm{MaStr}]$; see $[\mathrm{P}]$ for a recent work on this type of models.

The picture which emerges from Mayer's work and the subsequent papers mentioned above (see e.g. [KaU]) is very different from the previous one.

(1) The equation for an isotherm is derived solely from the partition function.

(2) A (first order) phase transition point corresponds to a singularity of the pressure.

(3) One must take the thermodynamic limit in order to have singularities and three different analytic parts for the isotherm.

(4) In the thermodynamic limit one cannot obtain states corresponding to supersaturated vapor states for example. Only equilibrium states are obtained.

These statements were not mathematically demonstrated when they were formulated. Importance of the thermodynamic limit was emphasized by

\footnotetext{
${ }^{12}$ Today we know that this is not correct.

${ }^{13}$ See in particular the excellent paper $[\mathrm{F}]$ and $[\mathrm{dB} 1]$ for a treatment of this model.
} 
Kramers at the van der Waals Centenary Congress, see [D]. He pointed out that one is really interested not in the partition function itself, but in the thermodynamic limit of the free energy. Only in this limit one may obtain non-analytic behaviour at certain densities and temperatures. In 1949 van Hove $[\mathrm{vH}]$ proved the existence ${ }^{14}$ of the thermodynamic limit, and Yang and Lee [YLe] demonstrated in 1952 how in the thermodynamic limit one may obtain singularities of the pressure, by accumulation on the real axis of complex zeros of the partition functions. However, these papers do not contain any information about the nature of the singularity of the pressure at a first order transition ${ }^{15}$; they do not settle Mayer's conjecture. Several arguments have been given in favor of Mayer's conjecture, or against it. None of them could give a definite answer. Indeed, either they are based on the droplet model (e.g. $[\mathrm{A}],[\mathrm{F}],[\mathrm{La} 1])$, or they rely on mean-field approximation (e.g. [Kat1], [Kat2], [T]).

\subsection{The van der Waals limit}

In the beginning of the fifties, the orthodox view was that van der Waals theory was merely an extrapolation from the first two terms of the virial series, and that the equal area construction an ex post facto introduction of thermodynamics, which would not be necessary if one could actually evaluate the partition function exactly, and obtain from it the pressure in the thermodynamic limit. In this context a remarkable achievement of Mathematical Physics is the derivation of the van der Waals-Maxwell isotherms from Statistical Mechanics only, in the limiting case of infinitely long-range and infinitely weak interactions ${ }^{16}$. Brout in [Bro] studied the Ising model in this limit, in relation with the mean-field theory. He tried to develop a perturbation around the mean-field limit. He showed how one can recover this limiting case by taking the limit of infinitely long-range and infinitely weak interactions, so that the overall strength of the interaction is constant. Baker also studied a similar limiting case for a one-dimensional spin system $[\mathrm{Ba}]$. However, the derivation of van der Waals equation in this limit is due to Kac, Hemmer and Uhlenbeck in [KUH1], [KUH3] and [KUH3] for a one-dimensional model of $N$ particles in an interval of length

\footnotetext{
${ }^{14}$ See Ruelle for further results [Ru].

${ }^{15}$ Chapter 15 of [Hu] (German edition (1964)) is an excellent exposition of these fundamental results obtained by van Hove and Yang and Lee. See also chapter two of [UF]. The main result in [YLe] is that, if a region of the complex plane is free of zeros of the partition functions, then the pressure is analytic in that region. Accumulation of the zeros of the partition functions is a necessary, but not sufficient condition for the existence of a singularity of the pressure. See [Sh] for examples of accumulation of the zeros on some points of the real axis, without producing a singularity of the pressure. For the mean-field Ising model there is accumulation of the zeros of the partition functions at $h=0$, when the temperature is low enough, since the pressure is not analytic in the thermodynamic limit. But in this case, contrary to Isakov's theorem, there is an analytic continuation of the pressure at $h=0$.

${ }^{16}$ Systems with weak long-range potentials are reviewed in [HLeb]. See also [Leb].
} 
$L$, with hard-core of size $\delta>0$ and interacting via an attractive interaction

$$
-a \gamma \mathrm{e}^{-\gamma r} \text {. }
$$

For finite $\gamma$ the model does not exhibit a phase transition, since it is a one-dimensional model with exponentially decaying interaction. However, if one takes the limit $\gamma$ tending to 0 , the so-called van der Waals limit, after the thermodynamic limit, then appears a phase transition, which is described by Gibbs' construction, so that the isotherms are given by

$$
\left(p+\frac{a}{l^{2}}\right)(l-\delta)=k T
$$

and Maxwell's rule. In 1964 van Kampen gave a derivation of van der Waals equation together with Maxwell's rule [vK]. The arguments of van Kampen are "local mean-field" type arguments. The basic idea is that there are two scales. The system is divided into large cells, which are small compared to the range of the attractive interaction, but large enough in order to contain many particles, and such that inside a cell one can use a meanfield approximation. In this way van Kampen obtained a coarse-grained description of the model. The distribution of the particles is uniform over a cell, but not over the whole system. The system can be partly in a gaseous phase or partly in a liquid phase, and one can define a free energy for a given non-homogeneous coarse-grained distribution, which is essentially the sum of the free energies of the cells. The equilibrium free energy of the whole system is obtained by minimizing the free energy over non-homogeneous coarse-grained distributions. Similar ideas are developed in section 5.

Lebowitz and Penrose [LebP], inspired by the ideas of van Kampen, proved the following remarkable result. Let $\varsigma: \mathbb{R}^{d} \rightarrow \mathbb{R}, \varsigma(x)=\varsigma(|x|)$ be a (positive) function with compact support in $[-1,1]^{d}$, so that

$$
\int \varsigma(x) d x=\alpha>0 .
$$

Let $0<\gamma<1$. The interaction potential between particles located at $x \in \mathbb{R}^{d}$ and $y \in \mathbb{R}^{d}$ is given by

$$
\phi_{\gamma}(|x-y|)=q(|x-y|)-\gamma^{d} \varsigma(\gamma|x-y|),
$$

where $q(|x|)$ is a fixed short-range repulsive potential which diverges at the origin more rapidly than $|x|^{-d^{\prime}}, d^{\prime}>d$. If the interaction potential is $q$ only (reference system), then the free energy (at given temperature and in the thermodynamic limit) is $\tilde{f}(\rho)$, whereas the free energy (in the thermodynamic limit) for the full interaction potential $\phi_{\gamma}$ is denoted by $f_{\gamma}(\rho)$. By general results $f_{\gamma}(\rho)$ is convex. Therefore, as one takes the van der Waals limit $\gamma \rightarrow 0$, the limiting free energy remains convex. However, this limiting convex free energy is the convex envelope of the non-convex free energy

$$
-\frac{1}{2} \alpha \rho^{2}+\tilde{f}(\rho),
$$


that is, in the van der Waals limit $\gamma \rightarrow 0$,

$$
\lim _{\gamma \rightarrow 0} f_{\gamma}(\rho)=\mathrm{CE}\left[-\frac{1}{2} \alpha \rho^{2}+\tilde{f}(\rho)\right] \quad(\mathrm{CE} \text { means convex envelope }) .
$$

\subsection{Lattice gas model, the Ising model}

Mayer's conjecture is still a completely open problem for models of simple fluids. It is reasonable and interesting to study this conjecture by considering simpler systems by putting the particles on the sites of the lattice $\mathbb{Z}^{d}$. For each $i \in \mathbb{Z}^{d}$ there is a variable $n_{i}=0,1$ with the interpretation that $n_{i}=1$ means presence of a particle at $i$, and $n_{i}=0$ means absence of a particle. There is at most one particle at a given site and the hamiltonian is

$$
-\sum_{\substack{\{i, j\} \subset \mathbb{Z}^{d} \\ i \neq j}} K(i-j) n_{i} n_{j}-\mu \sum_{i \in \mathbb{Z}^{d}} n_{i},
$$

with $K(i-j)=K(j-i)=K>0$ if the sites $i$ and $j$ are nearest neighbors, and $K(i-j)=0$ otherwise. The constant $\mu$ is the chemical potential; this term controls the density of the system. If one considers the pressure as a function of the particle density $\rho$ or as a function of the specific volume $v=\rho^{-1}$, then one gets isotherms qualitatively similar to the isotherms of a simple fluid (see $[\mathrm{LeY}]$ or $[\mathrm{Fr}]$ ). This model is equivalent to the Ising ferromagnetic model, which is one of the fundamental models of theoretical physics ${ }^{17}$. Formally, one gets the Ising model by introducing the spin variables $\sigma_{i}=2 n_{i}-1= \pm 1$. The hamiltonian becomes, up to a constant,

$$
-\sum_{\substack{\{i, j\} \subset \mathbb{Z}^{d} \\ i \neq j}} J(i-j) \sigma_{i} \sigma_{j}-h \sum_{i \in \mathbb{Z}^{d}} \sigma_{i} .
$$

The coupling constant is $J(i-j)=J(j-i)=4 K \equiv J$ if $i$ and $j$ are nearest neighbors, and $J(i-j)=0$ otherwise. The constant $h=2 \mu-2 d K$ is the external magnetic field. The magnetization $m$ is related to the density $\rho$ of the lattice gas. The Ising model has a transition point at $h=0$ and low temperatures. At this point the density of the vapor phase $\rho_{g}$ and of the liquid phase $\rho_{l}$ are given by

$$
\rho_{g}=\frac{1-m_{\star}}{2} \text { and } \quad \rho_{l}=\frac{1+m_{\star}}{2},
$$

\footnotetext{
${ }^{17}$ Several important methods or techniques in Mathematical Physics have been first developed for that model, e.g. Peierls' argument [Pe], or exact computations of partition functions [On]. The paper of Yang and Lee [YLe] about the general mechanism for the existence of singularities in the thermodynamic potentials was very convincing because they could prove their Circle Theorem for the zeros of the partition function of the Ising model [LeY].
} 
where $m_{\star}$ is the spontaneous magnetization. In this paper I choose to discuss the ferromagnetic Ising model, but I use the terminology pressure for the thermodynamic potential

$$
p(h, \beta):=\lim _{n \rightarrow \infty} \frac{1}{\beta\left|B_{n}(0)\right|} \ln \sum_{\substack{\sigma_{k}= \pm 1, \forall k \in B_{n}(0)}} \exp \left(-\beta H_{B_{n}(0)}\right),
$$

where

$$
H_{B_{n}(0)}:=-\sum_{\substack{\{i, j\} \subset B_{n}(0) \\ i \neq j}} J(i-j) \sigma_{i} \sigma_{j}-h \sum_{i \in B_{n}(0)} \sigma_{i},
$$

$\beta:=\frac{1}{k T}$ is the inverse temperature and $\left|B_{n}(0)\right|$ is the number of sites of the box $B_{n}(0):=\left\{i \in \mathbb{Z}^{d}:\left|i_{k}\right| \leq n, \forall k=1, \ldots, d\right\}$. In the lattice gas interpretation $p(h, \beta)$ corresponds to the grand canonical pressure. It is the quantity analogous ${ }^{18}$ to that considered by Mayer. The breakthrough for Mayer's conjecture came with the profound work of Isakov [I1] in 1984. Isakov proved Mayer's conjecture by ruling out the possibility of an analytic continuation at a first order phase transition point. For the first time a definite result was established.

Theorem 2.1 (Isakov). In dimension $d \geq 2$, at low enough temperature, the pressure of the Ising model in a magnetic field $h, p=p(h)$, is infinitely differentiable at $h=0^{ \pm}$, and for large $k$

$$
p^{(k)}\left(0^{ \pm}\right) \sim C^{k} k !^{\frac{d}{d-1}} .
$$

For the Ising model one can define two Taylor series of the pressure at $h=0$ by evaluating the derivatives at $h=0^{+}$, respectively $h=0^{-}$. Both series have zero convergence radius, so that there is no analytic continuation of $p$ from $\{h<0\}$ to $\{h>0\}$ across $h=0$, or vice versa. Isakov extended his result to generic two phase lattice models in [I2]. He had, however, to introduce technical hypotheses that are difficult to verify in concrete models. In [FrPf1] a genuine extension of Theorem 2.1 is proved for a large class of lattice models; see Theorem 4.1 section 4 for precise statements. Theorem 4.1 can be rephrased as follows in the setting of Pirogov-Sinai theory.

Consider a path in the phase diagram, which crosses transversally at point $P$ the manifold of coexistence of two phases. Then, at sufficiently low temperature the pressure along this path has no analytic continuation at $P$.

This proves Mayer's conjecture. Suppose that at $P$ the phases $A$ and $B$ coexist, and that the path starts inside the phase $A$. Then, by analytic

\footnotetext{
${ }^{18}$ More precisely, in Mayer's approach one chooses $p$ as a function of $z=\mathrm{e}^{h \beta}$ instead of $h$.
} 
continuation of the pressure along the path, one can determine the phase coexistence point $P$, as the first singularity of the pressure along the path.

These results are in particular true for ferromagnetic Kac-Ising models which are Ising models with coupling constants

$$
J_{\gamma}(x)=c_{\gamma} \gamma^{d} \varsigma(\gamma x), \quad(0<\gamma<1)
$$

and

$$
\varsigma(x):= \begin{cases}2^{-d} & \text { if } x \in[-1,1]^{d} ; \\ 0 & \text { otherwise. }\end{cases}
$$

The constant $c_{\gamma}$ in the definition of the interaction, whose range is $\gamma^{-1}$, is chosen so that

$$
\sum_{x \in \mathbb{Z}^{d}: x \neq 0} J_{\gamma}(x)=1 .
$$

By studying Kac-Ising models Friedli gave in his PhD-thesis an unexpectedly simple answer to the following original question: how the breakdown of analyticity at a first order phase transition point is restored in the van der Waals limit $\gamma \rightarrow 0$ ?

Theorem 2.2. For Kac-Ising models there exist $\gamma_{0}>0$ and $\beta_{\star}$, independent of $\gamma$, so that for any $\beta \geq \beta_{\star}$ and any $0<\gamma \leq \gamma_{0}$ the pressure $p_{\gamma}$ has no analytic continuation at the first order phase transition point $h=0$. Furthermore, there exists also a constant $C$, independent of $\gamma$ and $\beta$ so that

$$
\left|p_{\gamma}^{(k)}\left(0^{ \pm}\right)\right| \leq C^{k} k ! \quad \text { for all } k \leq k_{1}(\gamma, \beta) \text {, with } k_{1}(\gamma, \beta)=\gamma^{-d} \beta .
$$

The first part of Theorem 2.2 is not a consequence of above results, which apply to any Kac-Ising model, but only for $\beta \geq \beta_{\gamma}^{*}$, with $\lim _{\gamma \rightarrow 0} \beta_{\gamma}^{*}=\infty$. If $\beta \geq \beta_{\star}$, then there is a phase $\operatorname{transition}^{19}$ at $h=0$ for all $\gamma<\gamma_{0}$. The pressure $p_{\gamma}$ has no analytic continuation at the transition point as long as the range of interaction is finite $(\gamma>0)$. However, for long range and weak interactions the derivatives of the pressure of order smaller than $\gamma^{-d} \beta$ behave like the derivatives of an analytic function at the transition point. Analytic continuation occurs only after the van der Waals limit $(\gamma \rightarrow 0)$. One can prove similar results concerning the free energy $f_{\gamma}$ for given magnetization $m$, which is related to the pressure $p_{\gamma}$ by a Legendre transform. The free energy for given magnetization $m$ is

$$
f_{\gamma}(m, \beta)=\sup _{h}\left(h m-p_{\gamma}(h, \beta)\right) .
$$

In the van der Waals limit

$$
\lim _{\gamma \rightarrow 0} f_{\gamma}(m, \beta) \equiv f_{0}(m, \beta)=C E\left(f_{\mathrm{mf}}(m, \beta)\right) .
$$

${ }^{19} \mathrm{See}[\mathrm{CPr}]$ and $[\mathrm{BoZ} 1]$. The existence of phase transition is proved for $\beta>1$, provided that $\gamma$ is small enough. The mean-field model has a phase transition if and only if $\beta>1$. 


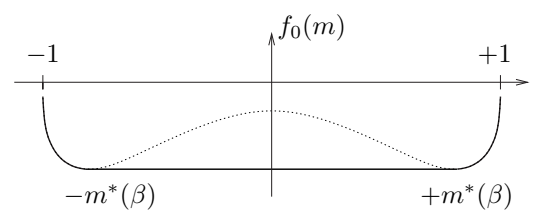

Figure 2: The free energy $f_{0}$ when $\beta>1$. The dotted line is the analytic continuation provided by $f_{\mathrm{mf}}$.

$f_{\mathrm{mf}}(m)$ is the mean-field free energy,

$$
f_{\mathrm{mf}}(m, \beta)=-\frac{1}{2} m^{2}-\frac{1}{\beta} I(m) \quad \text { with } m \in[-1,+1],
$$

where $I(m)$ is the entropy term,

$$
I(m):=-\frac{1-m}{2} \ln \frac{1-m}{2}-\frac{1+m}{2} \ln \frac{1+m}{2} .
$$

If $\beta \geq \beta_{\star}$ the free energy $f_{\gamma}$ is affine above $\left[-m^{*}(\beta, \gamma), m^{*}(\beta, \gamma)\right]$, with

$$
m^{*}(\beta, \gamma)=\left.\frac{d}{d h} p_{\gamma}(h, \beta)\right|_{h=0+}>0 .
$$

Theorem 2.3. There exists $\beta_{\star}$ and $\gamma_{0}>0$ such that for all $\beta \geq \beta_{\star}$, $\gamma \in\left(0, \gamma_{0}\right), f_{\gamma}$ is analytic at any $m \in(-1,+1)$, except at $\pm m^{*}(\beta, \gamma)$.

$f_{\gamma}$ has no analytic continuation beyond $-m^{*}(\beta, \gamma)$ along the real path $m<$ $-m^{*}(\beta, \gamma)$.

$f_{\gamma}$ has no analytic continuation beyond $m^{*}(\beta, \gamma)$ along the real path $m>$ $m^{*}(\beta, \gamma)$.

By contrast, after the van der Waals limit, $f_{0}=\lim _{\gamma \rightarrow 0} f_{\gamma}$ has a plateau for $m \in\left[-m^{*}(\beta),+m^{*}(\beta)\right]$, where $m^{*}(\beta)$ is the positive solution of the mean-field equation $m=\tanh (\beta m)$, and $f_{0}$ has analytic continuations beyond $\pm m^{*}(\beta)$, which are given by the mean-field free energy (2.2).

From the proofs of Theorems 2.2 and 2.3 one gets an even more refined understanding of the restoration of the analytic continuation (see subsection 5.6). A main property of mean-field models is that the states are homogeneous, as already mentioned in the case of the theory of van der Waals. There is no state describing the phenomenon of phase separation as in the Ising model. For small $\gamma$, in order to prevent this phenomenon, one proceeds as follows (see section 5 ). One fixes a small positive parameter $0<\delta<2^{-d}$. Let $i \in \mathbb{Z}^{d}$ and $B_{\gamma^{-1}}(i)$ be the box of linear size $2 \gamma^{-1}+1$ centered at $i$. In a spin configuration one says that the site $i$ is $(\delta,+)$-correct if 
the number of spins $\sigma_{j}=-1$ with $j \in B_{\gamma^{-1}}(i)$ is smaller than $\frac{\delta}{2}\left|B_{\gamma^{-1}}(i)\right|$. Similarly, in a spin configuration one says that the site $i$ is $(\delta,-)$-correct if the number of spins $\sigma_{j}=1$ with $j \in B_{\gamma^{-1}}(i)$ is smaller than $\frac{\delta}{2}\left|B_{\gamma^{-1}}(i)\right|$. A site is $\delta$-correct if it is either $(\delta,-)$-correct or $(\delta,+)$-correct. One can show that if all sites are $\delta$-correct, then they are all $(\delta,-)$-correct or they are all $(\delta,+)$-correct. Therefore, by considering only configurations with $\delta$-correct sites one prevents the occurrence of the phenomenon of phase separation. One defines a constrained partition function, by summing only over configurations with $\delta$-correct sites. From the constrained partition function one gets the constrained pressure $\widehat{p}_{\gamma}$. The constrained pressure $\widehat{p}_{\gamma}$ is convex and symmetric in $h$, and it has a phase transition point at $h=0$,

$$
\widehat{m}_{\gamma}^{*}(\beta):=\left.\frac{d}{d h} \widehat{p}_{\gamma}(h, \beta)\right|_{h=0+}>0 .
$$

The constrained pressure $\widehat{p}_{\gamma}$ is the main contribution to $p_{\gamma}$ in the following sense:

$$
\lim _{\gamma \rightarrow 0} \widehat{p}_{\gamma}(h, \beta)=\lim _{\gamma \rightarrow 0} p_{\gamma}(h, \beta)=p_{\mathrm{mf}}(h, \beta):=\sup _{m}\left(h m-f_{\mathrm{mf}}(m, \beta)\right),
$$

and

$$
\lim _{\gamma \rightarrow 0} \widehat{m}_{\gamma}^{*}(\beta)=m^{*}(\beta) \quad \text { (mean-field spontaneous magnetization) . }
$$

Using a Legendre transform one defines on $(-1,1)$ a constrained free energy

$$
\widehat{f}_{\gamma}(m, \beta):=\sup _{h}\left(h m-\widehat{p}_{\gamma}(h, \beta)\right) .
$$

The free energy $\widehat{f}_{\gamma}(m, \beta)$ is affine on the interval $\left[-\widehat{m}_{\gamma}^{*}(\beta), \widehat{m}_{\gamma}^{*}(\beta)\right]$, and

$$
\lim _{\gamma \rightarrow 0} \widehat{f}_{\gamma}(m, \beta)=C E\left(f_{\mathrm{mf}}(m, \beta)\right) .
$$

There is a major difference with the previous results. For large $\beta$ the constrained pressure $\widehat{p}_{\gamma}$ has an analytic continuation $\tilde{p}_{\gamma}^{+}$at $h=0$ from $h>0$ to $h>-1 / 8$, and of course also an analytic continuation $\widetilde{p}_{\gamma}^{-}$at $h=0$ from $h<0$ to $h<1 / 8$ (Theorem 5.3). The analytic continuation of the constrained pressure from $h>0$ to $h>-1 / 8$ is convex and in the van der Waals limit it coincides with the analytic continuation of the meanfield pressure. By a Legendre transform one can also define an analytic continuation $\widetilde{f}_{\gamma}^{+}$of the constrained free energy at $\widehat{m}_{\gamma}^{*}(\beta)$ from $m>\widehat{m}_{\gamma}^{*}(\beta)$ to $m>m^{\prime}$, with $m^{\prime}$ is independent of $\gamma$ and $m^{\prime}<\widehat{m}_{\gamma}^{*}(\beta)$.

In conclusion, when the phenomenon of phase separation is prevented the constrained pressure has an analytic continuation at $h=0$. It is the term

$$
\operatorname{sing}_{\gamma}(h, \beta):=p_{\gamma}(h, \beta)-\widehat{p}_{\gamma}(h, \beta)
$$


which is responsible for the absence of analytic continuation of $p_{\gamma}$ at $h=0$. The term $\operatorname{sing}_{\gamma}$ gives the contribution to $p_{\gamma}$ of the configurations where both $(\delta,+)$-correct and $(\delta,-)$-correct points occur. It is a small term since

$$
\left|\operatorname{sing}_{\gamma}(h, \beta)\right| \leq a \mathrm{e}^{-b \beta \gamma^{-d}}, \quad|h| \leq h^{*},
$$

for some positive constants $h^{*}, a$ and $b$. On the other hand there exists a diverging sequence of integers $k_{i}$, so that

$$
\left.\frac{d^{k_{i}}}{d h^{k_{i}}} \operatorname{sing}_{\gamma}(h, \beta)\right|_{h=0 \pm} \sim C_{ \pm}^{k_{i}} k_{i} !^{\frac{d}{d-1}}
$$

\section{Phase transitions in lattice models at low temperatures}

Pirogov and Sinai [PiSi], [Si], have constructed, at low temperature, the phase diagram of all lattice models with a finite number of ground-states verifying Peierls' condition. In this section I consider lattice models with only two ground-states in the low temperature regime and construct the phase diagram of such models when Peierls' condition is verified. The notations are similar to those of Sinai [Si] chapter II, so that it is easy to consult this text for further details, if necessary. The construction of the phase diagram relies on the original work of Isakov [I2].

\subsection{Lattice models, main assumptions}

A lattice model is defined by specifying a lattice and a potential. The usual choice for the lattice is

$$
\mathbb{Z}^{d}:=\{x=(x(1), \ldots, x(d)): x(i) \in \mathbb{Z}\} \quad \text { with } \quad d \geq 2,
$$

which is equipped with a norm,

$$
|x|:=\max _{i=1}^{d}|x(i)| .
$$

For finite $R>0$, let

$$
B_{R}(x):=\left\{y \in \mathbb{Z}^{d}:|x-y| \leq R\right\} .
$$

The cardinality of a (finite) subset $C$ is denoted by $|C|$. Let $S$ be a finite set called the state space of the model. A configuration of the model is a function $\varphi: \mathbb{Z}^{d} \rightarrow S$. The restriction of $\varphi$ to $A \subset \mathbb{Z}^{d}$ is denoted by $\varphi(A)$, and two configurations $\varphi, \psi$ are almost surely equal, $\varphi=\psi$ a.s., if $|\{x: \varphi(x) \neq \psi(x)\}|$ is finite. The set of configurations is $\Omega$, and there is a natural action of $\mathbb{Z}^{d}$ on $\Omega$,

$$
\left(T^{y} \varphi\right)(x):=\varphi(x-y), \quad y \in \mathbb{Z}^{d} .
$$


The interaction between spins are defined by a potential, which is a family of real-valued functions $\Phi_{A}$ defined on $\Omega$ and indexed by the finite subsets of $\mathbb{Z}^{d}$. These functions are local and the potential is summable, which means that

$$
\forall A, \Phi_{A}(\varphi)=\Phi_{A}(\psi) \text { if } \varphi(A)=\psi(A) \text { and } \sum_{A \ni 0} \sup _{\varphi \in \Omega}\left|\Phi_{A}(\varphi)\right|<\infty .
$$

I consider only interactions of finite range, that is, I assume the existence of $R<\infty$ such that

$$
\Phi_{A} \equiv 0 \text { if } \nexists a \in \mathbb{Z}^{d} \text { such that } A \subset B_{R}(a) .
$$

Let $\mathbb{Z}_{0}^{d}$ be a subgroup of $\mathbb{Z}^{d}$ such that the quotient group $\mathbb{Z}^{2} / \mathbb{Z}_{0}^{d}$ is a finite group. The potential is periodic, or $\mathbb{Z}_{0}^{d}$-periodic, if

$$
\Phi_{A}(\varphi)=\Phi_{A+y}\left(T^{y} \varphi\right) \quad \forall \varphi, \forall A, \forall y \in \mathbb{Z}_{0}^{d} .
$$

In this context the main quantity is the pressure of the model, at inverse temperature $\beta>0$, which is defined by

$$
p(\beta):=\lim _{r \rightarrow \infty} \frac{1}{\beta\left|B_{r}(0)\right|} \ln \left(\sum_{\varphi\left(B_{r}(0)\right)} \exp \left[-\beta \sum_{A \subset B_{r}(0)} \Phi_{A}\left(\varphi\left(B_{r}(0)\right)\right]\right) .\right.
$$

It is convenient to introduce

$$
\mathcal{U}_{x}:=\sum_{A \ni x} \frac{1}{|A|} \Phi_{A}
$$

and to define in this section the hamiltonian $\mathcal{H}$ as the formal sum $\mathcal{H}=$ $\sum_{x \in \mathbb{Z}^{d}} \mathcal{U}_{x}$. For two configurations $\varphi$ and $\psi$, such that $\varphi=\psi$ (a.s.), the relative hamiltonian

$$
\mathcal{H}(\varphi \mid \psi):=\sum_{x \in \mathbb{Z}^{d}}\left(\mathcal{U}_{x}(\varphi)-\mathcal{U}_{x}(\psi)\right)
$$

is a sum with only a finite number of nonzero terms since the interaction has finite range. A configuration $\psi$ is a ground-state of $\mathcal{H}$ if

$$
\mathcal{H}(\varphi \mid \psi) \geq 0 \quad \text { whenever } \varphi=\psi \text { a.s. . }
$$

For a periodic configuration $\varphi$ the specific energy of the configuration is well-defined,

$$
h(\varphi)=\lim _{r \rightarrow \infty} \frac{1}{\left|B_{r}(0)\right|} \sum_{x \in B_{r}(0)} \mathcal{U}_{x}(\varphi) .
$$

A periodic ground-state can be characterized as a periodic configuration with minimal specific energy (see [Si] p.36). 
Let $\mathcal{H}_{0}$ be a hamiltonian with a $\mathbb{Z}_{0}^{d}$-periodic interaction of finite range $R$,

$$
\mathcal{H}_{0}=\sum_{x \in \mathbb{Z}^{d}} \mathcal{U}_{0, x},
$$

such that $\mathcal{H}_{0}$ has two $\mathbb{Z}_{0}^{d}$-periodic ground-states denoted by $\psi_{1}$ and $\psi_{2}$. Let $s$ be an integer, such that $s \geq R$ and $s \geq\left|\mathbb{Z}^{d} / \mathbb{Z}_{0}^{d}\right|$.

Definition 3.1. Given $\varphi$, a lattice site $x$ is $\psi_{j}$-correct if

$$
\varphi\left(B_{s}(x)\right)=\psi_{j}\left(B_{s}(x)\right) .
$$

It is correct if it is $\psi_{1}$-correct or $\psi_{2}$-correct, otherwise it is incorrect.

The boundary of a configuration $\varphi$ is by definition the subset of $\mathbb{Z}^{d}$

$$
\partial \varphi:=\bigcup_{\substack{x \in \mathbb{Z}^{d}: x \\ \text { incorrect for } \varphi}} B_{s}(x) .
$$

Assumption I. The ground-states $\psi_{m}$ of $\mathcal{H}_{0}, m=1,2$, verify Peierls' condition, that is, there exists a constant $\rho>0$ such that

$$
\mathcal{H}_{0}\left(\varphi \mid \psi_{m}\right) \geq \rho|\partial \varphi| \quad \forall \varphi, \varphi=\psi_{m} \text { a.s. }
$$

Peierls' condition is a very natural assumption. It means that in order to create a boundary one needs an energy at least proportional to the size of the boundary. Boundaries are locations of energy barriers.

Let $\mathcal{H}_{1}$ be another hamiltonian with a $\mathbb{Z}_{0}^{d}$-periodic interaction of finite range $R$,

$$
\mathcal{H}_{1}(\varphi)=\sum_{x \in \mathbb{Z}^{d}} \mathcal{U}_{1, x} .
$$

The hamiltonian of the model, $\mathcal{H}^{\mu}$, is the sum of $\mathcal{H}_{0}$ and $\mu \mathcal{H}_{1}$,

$$
\mathcal{H}^{\mu}:=\mathcal{H}_{0}+\mu \mathcal{H}_{1}, \quad \mu \in \mathbb{R} .
$$

Assumption II. $\mathcal{H}_{1}$ splits the degeneracy of the ground-states of $\mathcal{H}_{0}$, that is, there exists $\varepsilon>0$ such that if $\mu \in(-\varepsilon, 0), \mathcal{H}^{\mu}$ has a unique ground-state, which is $\psi_{2}$; if $\mu \in(0, \varepsilon), \mathcal{H}^{\mu}$ has a unique ground-state, which is $\psi_{1}$.

Example 1. Ising model.

The state space is $S=\{-1,1\}$. The hamiltonian $\mathcal{H}_{0}$ is given by the potential $\left\{\Phi_{A}\right\}$ such that $\Phi_{A} \equiv 0$ for all $A$ except if $A=\{x, y\}$ with $\sum_{i=1}^{d}|x(i)-y(i)|=1$, in which case

$$
\Phi_{\{x, y\}}(\varphi)=-J \frac{\varphi(x) \varphi(y)}{2} .
$$


The potential is $\mathbb{Z}^{d}$-invariant, $R=1$, and there are two ground-states, $\psi_{1}$ with $\psi_{1}(x)=1$ for all $x$, and $\psi_{2}$ with $\psi_{2}(x)=-1$ for all $x$. It is easy to verify Peierls' condition.

Let $\mathcal{H}_{1}$ be the hamiltonian given by the potential $\left\{\Phi_{A}^{\prime}\right\}$ such that $\Phi_{A}^{\prime} \equiv 0$ for all $A$ except if $A=\{x\}$, in which case

$$
\Phi_{\{x\}}^{\prime}(\varphi)=-\varphi(x) .
$$

The hamiltonian $\mathcal{H}_{1}$ split the degeneracy of the ground-states of $\mathcal{H}_{0}$. The hamiltonian $\mathcal{H}^{\mu}=\mathcal{H}_{0}+\mu \mathcal{H}_{1}$ is the hamiltonian of the Ising model with external magnetic field $\mu$ (denoted elsewhere by $h$ ).

\section{Example 2. Blume-Capel model.}

The state space is $\{-1,0,1\}$. Let $\lambda$ and $h$ be two real parameters. The hamiltonian $\mathcal{H}^{\lambda, h}$ is given by the potential $\left\{\Phi_{A}\right\}$ such that $\Phi_{A} \equiv 0$ for all $A$ except if $A=\{x, y\}$ with $\sum_{i=1}^{d}|x(i)-y(i)|=1$, in which case

$$
\Phi_{\{x, y\}}(\varphi)=\frac{(\varphi(x)-\varphi(y))^{2}}{2},
$$

or $A=\{x\}$ and

$$
\Phi_{\{x\}}(\varphi)=-\lambda \varphi(x)^{2}-h \varphi(x) .
$$

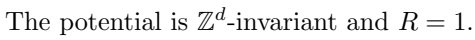

(a) If $\lambda>0$ and $h=0$, then the hamiltonian $\mathcal{H}_{0}=\mathcal{H}^{\lambda, 0}$ has two groundstates, which are $\psi_{1}$ and $\psi_{2}$ with $\psi_{1}(x) \equiv 1$ and $\psi_{2}(x) \equiv-1$. The hamiltonian $\mathcal{H}_{1}=\mathcal{H}^{0,1}-\mathcal{H}^{0,0}$ splits the degeneracy of the ground-states of $\mathcal{H}_{0}$. (b) If $\lambda<0$ and $h=-\lambda$, then the hamiltonian $\mathcal{H}_{0}=\mathcal{H}^{\lambda,-\lambda}$ has two ground-states, which are $\psi_{1}$ and $\psi_{3}$ with $\psi_{3}(x) \equiv 0$. The hamiltonian $\mathcal{H}_{1}=\mathcal{H}^{0,1}-\mathcal{H}^{0,0}$ splits the degeneracy of the ground-states of $\mathcal{H}_{0}$. The same is true for the hamiltonian $\mathcal{H}_{1}=\mathcal{H}^{1,0}-\mathcal{H}^{0,0}$. Similar statements hold in the case $\lambda<0$ and $h=\lambda$.

In both cases it is easy to verify Peierls' condition. Notice that the hamiltonian $\mathcal{H}^{0,0}$ has three ground-states, $\psi_{1}, \psi_{2}$ and $\psi_{3}$. Because of that, the constant $\rho$ in Peierls' condition for $\mathcal{H}^{\lambda,-\lambda}$ is bad, i.e. small, when $\lambda$ is small.

Let assumptions I and II be satisfied and let $p(\mu, \beta)$ be the pressure of the hamiltonian $\mathcal{H}^{\mu}=\mathcal{H}_{0}+\mu \mathcal{H}_{1}$. For $\mu<0$, respectively $\mu>0$, the unique ground-state is $\psi_{2}$, respectively $\psi_{1}$. Therefore, writing for a periodic configuration $h^{\mu}(\varphi) \equiv h_{0}(\varphi)+\mu h_{1}(\varphi)$, one gets

$$
h_{1}\left(\psi_{2}\right)-h_{1}\left(\psi_{1}\right)>0 \text {. }
$$

The quantity $\mathcal{U}_{1, x}$ is interpreted as an order parameter. The ground-state energy of $\mathcal{H}^{\mu}$, which is given by

$$
h(\mu):= \begin{cases}h_{0}\left(\psi_{2}\right)+\mu h_{1}\left(\psi_{2}\right) & \text { if } \mu \leq 0 \\ h_{0}\left(\psi_{1}\right)+\mu h_{1}\left(\psi_{1}\right) & \text { if } \mu \geq 0\end{cases}
$$


is a continuous function of $\mu$, which is analytic for $\mu<0$ and $\mu>0$, and has a kink at $\mu=0$. The main result of Pirogov-Sinai theory is that similar properties are true for the pressure $p(\mu, \beta)$ at low temperature.

Theorem 3.1. If the assumptions $I$ and II are satisfied, then there exist an open interval $\left(-\varepsilon^{\prime}, \varepsilon^{\prime}\right)$ and $\beta^{*}<\infty$ such that for any $\beta \geq \beta^{*}$ there exists a unique $\mu^{*}(\beta) \in\left(-\varepsilon^{\prime}, \varepsilon^{\prime}\right)$ with the following properties.

1. The pressure is not differentiable with respect to $\mu$, at $\mu=\mu^{*}(\beta)$ : left and right derivatives of $p(\mu, \beta)$ with respect to $\mu$, at $\mu^{*}(\beta)$, exist, but are different.

2. The pressure $p(\mu, \beta)$ is real-analytic in $\mu$ in $\left(-\varepsilon^{\prime}, \mu^{*}(\beta)\right)$.

3. The pressure $p(\mu, \beta)$ is real-analytic in $\mu$ in $\left(\mu^{*}(\beta), \varepsilon^{\prime}\right)$.

Theorem 3.1 establishes the existence of a first-order phase transition at $\mu^{*}(\beta)$. It is a consequence of Proposition 3.1. The mechanism for phase coexistence at $\mu^{*}(\beta)$ is described below.

Local perturbations of ground-states are described by contours, which are the connected parts of the boundaries of configurations. At low temperature, locally, a configuration coincides with high probability with one of the ground-state configurations, and all contours are finite geometrical objects. The contours describing perturbations of the (infinitely extended) ground-state $\psi_{1}$ are called $\psi_{1}$-contours; they differ in general from those describing perturbations of the ground-state $\psi_{2}$, called $\psi_{2}$-contours. The $\psi_{1}$-phase is stable if and only if all $\psi_{1}$-contours are stable (see Definition 3.7). Stability of all $\psi_{1}$-contours implies that the ground-state is stable with respect to local perturbations of any size, i.e. the ground-state for the infinitely extended system is stable. This is the origin of the $\psi_{1}$-phase.

If the $\psi_{1}$-phase is the only stable phase, then large $\psi_{2}$-contours are not stable. More generally, if one considers a given region $\mathcal{R}$ of the groundstate $\psi_{j}$, then this region of the ground-state $\psi_{j}$ is stable if and only if all $\psi_{j}$-contours inside $\mathcal{R}$ are stable. Inside any given region $\mathcal{R}$ all possible perturbations occur with nonzero probability, and the only way to stabilize a region $\mathcal{R}$ of the ground-state $\psi_{j}$, when all $\psi_{j}$-contours inside $\mathcal{R}$ are not stable, is to suppress the unstable contours. This is the basic idea of Zahradník in his fundamental paper [Z].

The geometry of contours is complex. Typically $\psi_{1}$-contours contain in their interiors $\psi_{2}$-contours and vice-versa. Suppose that the $\psi_{1}$-phase is stable and consider a given $\psi_{1}$-contour, which is by assumption stable. If all $\psi_{2}$-contours inside this contour are also stable, then the following is true: whenever this $\psi_{1}$-contour occurs, inside this contour the configuration is with high probability a small perturbation of the ground-state $\psi_{2}$, i.e. with high probability the configuration inside this $\psi_{1}$-contour is the ground-state configuration $\psi_{2}$ or a configuration which is almost the ground-state configuration $\psi_{2}$. In other words the ground-state $\psi_{2}$ is stable 
inside this $\psi_{1}$-contour. Of course, larger stable $\psi_{2}$-contours allows larger regions where the ground-state $\psi_{2}$ becomes stable. As one approaches the point of coexistence with the other phase (associated with ground-state $\psi_{2}$ ), more and more $\psi_{2}$-contours become stable ${ }^{20}$. It is precisely, when all contours of both phases become stable that there is coexistence of the two phases. This happens at well-defined values of $\beta$ and $\mu^{*}(\beta)$. The system knows when condensation takes place. The stability of contours is a consequence of a delicate balance between volume versus surface effects. The subtle question of non-existence of an analytic continuation of the pressure at a first order phase transition point is also related to the stability/instability properties of the contours of both phases in a (complex) neighbourhood of the coexistence point. Theorem 3.1 is proved by showing that for $\mu<\mu^{*}(\beta)$ the only stable phase is the $\psi_{2}$-phase, and that the only stable phase for $\mu>\mu^{*}(\beta)$ is the $\psi_{1}$-phase. At $\mu^{*}(\beta)$ both phases are stable. In the next subsections 3.2 and 3.3 this picture is made mathematically precise.

\subsection{Lattice models as contour models}

The re-formulation of the model as a contour model is an essential step in Pirogov-Sinai theory, which rests on few basic concepts: $(a)$ the notion of contour, together with the notion of weight of contour, $(b)$ the notion of stability of contour, (c) Peierls' condition. Technically, the basic formula is (3.4), which, together with Peierls' condition and Lemma 3.1, allows to establish stability of a contour. The idea of a contour model is to obtain a representation of the partition function $\Theta_{q}(\Lambda)$ (Definition 3.5) in terms of the contours, which interact only through a hard-core condition. I follow [Si].

To simplify slightly the exposition ${ }^{21}$, I assume from now on that the range of interaction is $R=1$, there are two translation invariant ground-states and that the specific energy of the ground-states for the hamiltonian $\mathcal{H}_{0}$ is given by

$$
\lim _{\Lambda \uparrow \mathbb{Z}^{d}} \frac{1}{|\Lambda|} \sum_{x \in \Lambda} \mathcal{U}_{0, x}\left(\psi_{m}\right)=\mathcal{U}_{0, y}\left(\psi_{m}\right)=0, \quad \forall y, m=1,2 .
$$

\footnotetext{
${ }^{20}$ There is an analogy with the mechanism of condensation in the droplet model. The title of [Fre2] is A General Theory of Heterophase Fluctuations and Pretransition Phenomena. From the abstract: [The paper] is based on the idea that the macroscopic transition of a substance from a phase $A$ to a phase $B$ is preceded by the formation of small nuclei being treated as resulting from "heterophase" density fluctuations or as manifestations of a generalized statistical equilibrium in which they play the roles of dissolved particles, whereas the A phase can be considered as the solvent. The heterophase or heterogeneous fluctuations should be contrasted with the ordinary density fluctuations.

${ }^{21}$ See the computation in (3.4). This is not a genuine restriction, since one can always, by an appropriate change of the lattice and of the state space $S$, reduce the general case to the case considered in these lectures. The price to pay is that $\beta^{*}$ in Theorem 3.1 could become much larger.
} 
Similarly, the specific energy of $\psi_{m}$ for the hamiltonian $\mathcal{H}_{1}$ is

$$
h_{1}\left(\psi_{m}\right):=\lim _{\Lambda \uparrow \mathbb{Z}^{d}} \frac{1}{|\Lambda|} \sum_{x \in \Lambda} \mathcal{U}_{1, x}\left(\psi_{m}\right)=\mathcal{U}_{1, y}\left(\psi_{m}\right), \quad \forall y, m=1,2 .
$$

Assumption II about the splitting of the ground-states of $\mathcal{H}_{0}$ by $\mathcal{H}_{1}$ implies that

$$
\Delta:=h_{1}\left(\psi_{2}\right)-h_{1}\left(\psi_{1}\right)>0 .
$$

Definition 3.2. Let $M$ denote a finite connected ${ }^{22}$ subset of $\mathbb{Z}^{d}$, and $\varphi$ a configuration. A couple $\Gamma=(M, \varphi(M))$ is called a contour of the configuration $\varphi$ if $M$ is a component of the boundary $\partial \varphi$. A couple $\Gamma=$ $(M, \varphi(M))$ is a contour if there exists a configuration such that $\Gamma$ is a contour of that configuration.

The subset $M$ of $\Gamma=(M, \varphi(M))$ is the support ${ }^{23}$ of the contour, and is denoted by supp $\Gamma$, or simply by $\Gamma$ when no confusion arises. In particular I use

$$
|\Gamma| \equiv|\operatorname{supp} \Gamma|
$$

Let $A_{\alpha}$ be the components of $\mathbb{Z}^{d} \backslash M$. For each component $A_{\alpha}$ there exists a unique label $q(\alpha) \in\{1,2\}$ such that

$$
\varphi_{\Gamma}(x):= \begin{cases}\psi_{q(\alpha)}(x) & \text { if } x \in A_{\alpha} \\ \varphi(x) & \text { if } x \in M\end{cases}
$$

is the unique configuration with the property that $\partial \varphi_{\Gamma}=M$ and $\varphi_{\Gamma}(M)=$ $\varphi(M)$. There is only one infinite component $A_{\alpha}$, called exterior of $\Gamma$, which is denoted by Ext $\Gamma$. All other components are the internal components; Int $_{m} \Gamma$ is the union of all internal components of $\Gamma$ with label $m$; the interior of $\Gamma$ is $\operatorname{Int} \Gamma:=\bigcup_{m=1,2} \operatorname{Int}_{m} \Gamma$. In order to indicate the label of Ext $\Gamma$, a superscript is added to $\Gamma$. Thus, $\Gamma^{q}$ means that on Ext $\Gamma$ the configuration $\varphi_{\Gamma}$ is equal to the ground-state configuration $\psi_{q} . \Gamma^{q}$ is a contour with boundary condition $\psi_{q}$, or $\psi_{q}$-contour. By definition, the volume of a contour $\Gamma^{q}$, with boundary condition $\psi_{q}$, is the total volume ${ }^{24}$ of the internal components of $\Gamma^{q}$ with label $m, m \neq q$ :

$$
V\left(\Gamma^{q}\right):=\left|\operatorname{Int}_{m} \Gamma^{q}\right| \quad(m \neq q) .
$$

Definition 3.3. Let $\Lambda \subset \mathbb{Z}^{d}$. A contour $\Gamma$ is inside $\Lambda$, which is written $\Gamma \subset \Lambda$, if $\operatorname{supp} \Gamma \subset \Lambda$, Int $\Gamma \subset \Lambda$ and ${ }^{25} d\left(\operatorname{supp} \Gamma, \Lambda^{c}\right)>1$. A contour $\Gamma$

\footnotetext{
${ }^{22} \mathrm{~A}$ path on $\mathbb{Z}^{d}$ is a set of points $\left\{x_{0}, x_{1}, \ldots, x_{n}\right\}$ with the property that $\left|x_{i}-x_{i-1}\right|=1$ for all $i=1, \ldots, n$. Connected set means path-connected set, and a component $B$ of a subset $A \subset \mathbb{Z}^{d}$ is a maximally path-connected subset of $A$.

${ }^{23}$ Thus, strictly speaking, at the end of subsection 3.1 one should say supports of contours instead of contours.

${ }^{24} \mathrm{My}$ convention differs here from that of Sinai [Si].

${ }^{25}$ If $A \subset \mathbb{Z}^{d}, B \subset \mathbb{Z}^{d}$, then $d(A, B):=\min _{x \in A} \min _{y \in B}|x-y|$.
} 
of a configuration $\varphi$ is an external contour of $\varphi$ if $\operatorname{supp} \Gamma \subset \operatorname{Ext} \Gamma^{\prime}$ for any other contour $\Gamma^{\prime}$ of $\varphi$. A compatible family of contours in $\Lambda$ is a family of contours with the same boundary condition, say $\left\{\Gamma_{1}^{q}, \ldots, \Gamma_{n}^{q}\right\}$, with $\Gamma_{i}^{q} \subset \Lambda$ and $d\left(\operatorname{supp} \Gamma_{i}^{q}, \operatorname{supp} \Gamma_{j}^{q}\right)>1$ for all $i \neq j$.

The basic statistical mechanical quantities of the theory are:

(1) the partition function $\Theta\left(\Gamma^{q}\right)$ of the contour $\Gamma^{q}$

(2) the partition function $\Theta_{q}(\Lambda)$ of the system in $\Lambda$, with boundary condition $\psi_{q}$,

(3) the weight $\omega\left(\Gamma^{q}\right)$ of the contour $\Gamma^{q}$.

Definition 3.4. Let $\Omega\left(\Gamma^{q}\right)$ be the set of configurations $\varphi=\psi_{q}$ (a.s.) such that $\Gamma^{q}$ is the only external contour of $\varphi$. The partition function of $\Gamma^{q}$ is

$$
\Theta\left(\Gamma^{q}\right):=\sum_{\varphi \in \Omega\left(\Gamma^{q}\right)} \exp \left[-\beta \mathcal{H}\left(\varphi \mid \psi_{q}\right)\right] .
$$

Definition 3.5. Let $\Omega_{q}(\Lambda)$ be the set of configurations $\varphi=\psi_{q}$ (a.s.) such that $\Gamma \subset \Lambda$ whenever $\Gamma$ is a contour of $\varphi$. The partition function of the system in $\Lambda$, with boundary condition $\psi_{q}$, is

$$
\Theta_{q}(\Lambda):=\sum_{\varphi \in \Omega_{q}(\Lambda)} \exp \left[-\beta \mathcal{H}\left(\varphi \mid \psi_{q}\right)\right] .
$$

Definition 3.6. Let $\Gamma^{q}$ be a contour with boundary condition $\psi_{q}$. The weight $\omega\left(\Gamma^{q}\right)$ of $\Gamma^{q}$ is

$$
\omega\left(\Gamma^{q}\right):=\exp \left[-\beta \mathcal{H}\left(\varphi_{\Gamma^{q}} \mid \psi_{q}\right)\right] \frac{\Theta_{m}\left(\operatorname{Int}_{m} \Gamma^{q}\right)}{\Theta_{q}\left(\operatorname{Int}_{m} \Gamma^{q}\right)} \quad(m \neq q) .
$$

The (bare) surface energy of a contour $\Gamma^{q}$ is

$$
\left\|\Gamma^{q}\right\|:=\mathcal{H}_{0}\left(\varphi_{\Gamma^{q}} \mid \psi_{q}\right) .
$$

For each ground-state $\psi_{q}$ one defines a $\psi_{q}$-dependent pressure

$$
g_{q}:=\lim _{r \rightarrow \infty} \frac{1}{\beta\left|B_{r}(0)\right|} \ln \Theta_{q}\left(B_{r}(0)\right) .
$$

It is easy to verify the identity

$$
g_{q}=p+\mu h_{1}\left(\psi_{q}\right) .
$$

The pressure $p$ does not depend on $\psi_{q}$, contrary to $g_{q}$. The partition function $\Theta_{q}(\Lambda)$ is equal to

$$
\Theta_{q}(\Lambda)=\sum \prod_{i=1}^{n} \Theta\left(\Gamma_{i}^{q}\right)
$$


where the sum is over the set of all compatible families $\left\{\Gamma_{1}^{q}, \ldots, \Gamma_{n}^{q}\right\}$ of external contours in $\Lambda$. On the other hand

$$
\Theta\left(\Gamma^{q}\right)=\exp \left[-\beta \mathcal{H}\left(\varphi_{\Gamma^{q}} \mid \psi_{q}\right)\right] \prod_{m=1}^{2} \Theta_{m}\left(\operatorname{Int}_{m} \Gamma^{q}\right) .
$$

Replacing $\Theta\left(\Gamma_{i}^{q}\right)$ in (3.1) by its expression given by (3.2), taking into account Definition 3.6, and iterating this procedure, one obtains easily the final form of the partition function $\Theta_{q}(\Lambda)$, as the partition function of a contour model, i.e.

$$
\Theta_{q}(\Lambda)=1+\sum \prod_{i=1}^{n} \omega\left(\Gamma_{i}^{q}\right),
$$

the sum being over all compatible families of contours $\left\{\Gamma_{1}^{q}, \ldots, \Gamma_{n}^{q}\right\}$ with boundary condition $\psi_{q}$.

Let $\Gamma^{q}$ be a contour and $m \neq q$.

$$
\begin{aligned}
\mathcal{H}\left(\varphi_{\Gamma^{q}} \mid \psi_{q}\right)= & \sum_{x \in \mathbb{Z}^{d}}\left(\mathcal{U}_{0, x}\left(\varphi_{\Gamma^{q}}\right)+\mu \mathcal{U}_{1, x}\left(\varphi_{\Gamma^{q}}\right)-\mathcal{U}_{0, x}\left(\psi_{q}\right)-\mu \mathcal{U}_{1, x}\left(\psi_{q}\right)\right) \\
= & \mathcal{H}_{0}\left(\varphi_{\Gamma^{q}} \mid \psi_{q}\right)+\sum_{x \in \operatorname{supp} \Gamma^{q}} \mu\left(\mathcal{U}_{1, x}\left(\varphi_{\Gamma^{q}}\right)-\mathcal{U}_{1, x}\left(\psi_{q}\right)\right) \\
& +\sum_{x \in \operatorname{Int} \Gamma^{q}} \mu\left(\mathcal{U}_{1, x}\left(\varphi_{\Gamma^{q}}\right)-\mathcal{U}_{1, x}\left(\psi_{q}\right)\right) \\
= & \left\|\Gamma^{q}\right\|+\mu \sum_{x \in \operatorname{supp} \Gamma^{q}}\left(\mathcal{U}_{1, x}\left(\varphi_{\Gamma^{q}}\right)-\mathcal{U}_{1, x}\left(\psi_{q}\right)\right) \\
& +\mu\left(h_{1}\left(\psi_{m}\right)-h_{1}\left(\psi_{q}\right)\right) V\left(\Gamma^{q}\right) \\
\equiv & \left\|\Gamma^{q}\right\|+\mu a\left(\varphi_{\Gamma^{q}}\right)+\mu\left(h_{1}\left(\psi_{m}\right)-h_{1}\left(\psi_{q}\right)\right) V\left(\Gamma^{q}\right) .
\end{aligned}
$$

In $(3.4)$

$$
a\left(\varphi_{\Gamma^{q}}\right):=\sum_{x \in \operatorname{supp} \Gamma^{q}} \mathcal{U}_{1, x}\left(\varphi_{\Gamma^{q}}\right)-\mathcal{U}_{1, x}\left(\psi_{q}\right) .
$$

Since the interaction is bounded, there exists a constant $C_{1}$ so that

$$
\left|a\left(\varphi_{\Gamma^{q}}\right)\right| \leq C_{1}\left|\Gamma^{q}\right| .
$$

The surface energy $\left\|\Gamma^{q}\right\|$ is always strictly positive since Peierls' condition holds, and there exists a constant $C_{2}$, independent of $q$, such that

$$
\rho\left|\Gamma^{q}\right| \leq\left\|\Gamma^{q}\right\| \leq C_{2}\left|\Gamma^{q}\right| .
$$

Definition 3.7. The weight $\omega\left(\Gamma^{q}\right)$ is $\tau$-stable for $\Gamma^{q}$ if there exists $\tau>0$ such that

$$
\left|\omega\left(\Gamma^{q}\right)\right| \leq \exp \left(-\tau\left|\Gamma^{q}\right|\right) .
$$

A contour is stable if its weight is stable. 
The dominant terms of the weight $\omega\left(\Gamma^{q}\right)$, in the neighbourhood of $\mu=0$, are $\left\|\Gamma^{q}\right\|$, the bare surface energy of $\Gamma^{q}$, and $\mu\left(h_{1}\left(\psi_{m}\right)-h_{1}\left(\psi_{q}\right)\right) V\left(\Gamma^{q}\right)$, which is a volume term. Stability of the weight is true when surface terms dominate volume terms (see (3.4)). In the discussion of the stability of weights, the isoperimetric inequality

$$
\chi_{q} V\left(\Gamma^{q}\right)^{\frac{d-1}{d}} \leq\left\|\Gamma^{q}\right\|
$$

plays a central role ${ }^{26}$.

The construction of the phase diagram is done by considering constrained partition functions and constrained pressures involving only contours such that $V\left(\Gamma^{q}\right) \leq n, n \in \mathbb{N}$. The phase diagram is constructed for these constrained pressures, and then one takes the limit $n \rightarrow \infty$. For given $n$, $n=0,1, \ldots$, the weight $\omega_{n}\left(\Gamma^{q}\right)$ is defined by

$$
\omega_{n}\left(\Gamma^{q}\right):= \begin{cases}\omega\left(\Gamma^{q}\right) & \text { if } V\left(\Gamma^{q}\right) \leq n, \\ 0 & \text { otherwise. }\end{cases}
$$

Let $l(n)$ be defined on $\mathbb{N}$ by

$$
l(n):=C_{0}^{-1}\left\lceil 2 d n^{\frac{d-1}{d}}\right\rceil \quad n \geq 1 .
$$

This function has the property ${ }^{27}$ :

$$
V\left(\Gamma^{q}\right) \geq n \quad \Longrightarrow \quad\left|\Gamma^{q}\right| \geq l(n) .
$$

So, if the volume $V\left(\Gamma^{q}\right)$ of a contour is large, then its surface energy cannot be too small (see (3.6)). For $q=1,2$, one defines constrained partition functions $\Theta_{q}^{n}$ by equation $(3.3)$, using $\omega_{n}\left(\Gamma^{q}\right)$ instead of $\omega\left(\Gamma^{q}\right)$.

It is essential for latter purposes to replace the real parameter $\mu$ by a complex parameter $z$; provided that $\Theta_{q}^{n}(\Lambda)(z) \neq 0$ for all $\Lambda$,

$$
g_{q}^{n}(z):=\lim _{r \rightarrow \infty} \frac{1}{\beta\left|B_{r}(0)\right|} \ln \Theta_{q}^{n}\left(B_{r}(0)\right)(z),
$$

and

$$
p_{q}^{n}(z):=g_{q}^{n}(z)-z h_{1}\left(\psi_{q}\right) .
$$

\footnotetext{
${ }^{26}$ In fact one uses a slightly different inequality in section 4 , because one knows little about the value of the best constant $\chi_{q}$.

${ }^{27}$ Given $\Lambda \subset \mathbb{Z}^{d}$, one defines $\partial|\Lambda|$ as the $(d-1)$-volume of the boundary of the set in $\mathbb{R}^{d}$ which is the union of unit cubes centered at the points of $\Lambda$. One has

$$
2 d|\Lambda|^{\frac{d-1}{d}} \leq \partial|\Lambda| \quad \text { (isoperimetric inequality) } .
$$
}

The constant $C_{0}$ is such that, if $\Lambda=\operatorname{Int}_{m} \Gamma^{q}$ and $\partial V\left(\Gamma^{q}\right):=\partial|\Lambda|$, then

$$
\partial V\left(\Gamma^{q}\right) \leq C_{0}\left|\Gamma^{q}\right| .
$$


$p_{q}^{n}$ is the constrained pressure of order $n$ and boundary condition $\psi_{q}$. Contrary to $p$, it depends on the boundary condition. Lemma 3.1 gives basic estimates for the rest of the paper. The only hypothesis for this lemma is that the weights of the contours are $\tau$-stable.

Lemma 3.1. Let $\omega\left(\Gamma^{q}\right)$ be any complex weights, depending on a parameter $t$ (real or complex). The weight $\omega_{n}\left(\Gamma^{q}\right)$ is defined by (3.7). Then there exist $K<\infty$ and $\tau^{*}<\infty$ independent of $n$, so that for any $\tau \geq \tau^{*}$ the following holds.

(A) Suppose that the weights $\omega_{n}\left(\Gamma^{q}\right)$ are $\tau$-stable for all $\Gamma^{q}$, as well as the weights $\frac{d}{d t} \omega_{n}\left(\Gamma^{q}\right)$ and $\frac{d^{2}}{d t^{2}} \omega_{n}\left(\Gamma^{q}\right)$. Then

$$
\beta\left|\frac{d^{k}}{d t^{k}} g_{q}^{n}\right| \leq K \mathrm{e}^{-\tau} \quad k=0,1,2 .
$$

For all finite subsets $\Lambda \subset \mathbb{Z}^{d}$,

$$
\left|\frac{d^{k}}{d t^{k}} \ln \Theta_{q}^{n}(\Lambda)-\beta \frac{d^{k}}{d t^{k}} g_{q}^{n}\right| \Lambda|| \leq K \mathrm{e}^{-\tau} \partial|\Lambda| \quad k=0,1,2 .
$$

(B) Let $m \geq 1$ and $n \geq m$. If $\omega_{n}\left(\Gamma^{q}\right)=0$ for all $\Gamma^{q}$ such that $\left|\Gamma^{q}\right| \leq m$, then

$$
\beta\left|g_{q}^{n}\right| \leq\left(K \mathrm{e}^{-\tau}\right)^{m},
$$

and

$$
\beta\left|g_{q}^{n}-g_{q}^{m-1}\right| \leq\left(K \mathrm{e}^{-\tau}\right)^{l(m)} .
$$

(C) If the weights $\omega_{n}\left(\Gamma^{q}\right)$ are $\tau$-stable for all $\Gamma^{q}$ and all $n \geq 1$, then all these estimates hold for $g_{q}$ and $\Theta_{q}$ instead of $g_{q}^{n}$ and $\Theta_{q}^{n}$. Moreover,

$$
\lim _{n \rightarrow \infty} \frac{d^{k}}{d t^{k}} g_{q}^{n}=\frac{d^{k}}{d t^{k}} g_{q}, \quad k=0,1,2 .
$$

Proof. The proof is based on the standard cluster expansion method. I follow [Pf1] section 3. Let $\omega\left(\Gamma^{q}\right)$ be an arbitrary weight, verifying the only condition that it is $\tau$-stable for any $\Gamma^{q}$. The partition function $\Theta_{q}(\Lambda)$ is defined in (3.3) by

$$
\Theta_{q}(\Lambda)=1+\sum \prod_{i=1}^{n} \omega\left(\Gamma_{i}^{q}\right)
$$

where the sum is over all families of compatible contours $\left\{\Gamma_{1}^{q}, \ldots, \Gamma_{n}^{q}\right\}$ with boundary condition $\psi_{q}$ (see Definition 3.3). Let $^{28}$,

$$
\overline{\Gamma^{q}}:=\left\{x \in \mathbb{Z}^{d}: d\left(x, \operatorname{supp} \Gamma^{2}\right) \leq 1\right\} .
$$

There exists a constant $C_{5}$ such that $\left|\overline{\Gamma^{q}}\right| \leq C_{5}\left|\Gamma^{q}\right|$, and

$$
\left(\Gamma_{i}^{q} \text { and } \Gamma_{j}^{q} \text { not compatible }\right) \Longrightarrow\left(\operatorname{supp} \Gamma_{i}^{q} \cap \overline{\Gamma_{j}^{q}} \neq \emptyset\right) .
$$

\footnotetext{
${ }^{28} \operatorname{In}[\mathrm{Pf} 1] \overline{\Gamma^{q}}$ is denoted by $i\left(\Gamma^{q}\right)$. In section $4 i\left(\Gamma^{q}\right)$ has a different meaning.
} 
Let

$$
\varphi_{2}\left(\Gamma_{i}^{q}, \Gamma_{j}^{q}\right):= \begin{cases}0 & \text { if } \Gamma_{i}^{q} \text { and } \Gamma_{j}^{q} \text { compatible } \\ -1 & \text { if } \Gamma_{i}^{q} \text { and } \Gamma_{j}^{q} \text { not compatible. }\end{cases}
$$

If the weights of all contours with boundary condition $\psi_{q}$ are $\tau$-stable and if $\tau$ is large enough, then one can express the logarithm of $\Theta_{q}(\Lambda)$ as

$$
\begin{aligned}
\ln \Theta_{q}(\Lambda) & =\sum_{m \geq 1} \frac{1}{m !} \sum_{\Gamma_{1}^{q} \subset \Lambda} \cdots \sum_{\Gamma_{m}^{q} \subset \Lambda} \varphi_{m}^{T}\left(\Gamma_{1}^{q}, \ldots, \Gamma_{m}^{q}\right) \prod_{i=1}^{m} \omega\left(\Gamma_{i}^{q}\right) \\
& =\sum_{m \geq 1} \frac{1}{m !} \sum_{x \in \Lambda} \sum_{\substack{\Gamma_{1}^{q} \subset \Lambda \\
x \in \operatorname{supp} \Gamma_{1}^{q}}} \cdots \sum_{\Gamma_{m}^{q} \subset \Lambda} \frac{\varphi_{m}^{T}\left(\Gamma_{1}^{q}, \ldots, \Gamma_{m}^{q}\right)}{\left|\operatorname{supp} \Gamma_{1}^{q}\right|} \prod_{i=1}^{m} \omega\left(\Gamma_{i}^{q}\right) .
\end{aligned}
$$

In (3.10) $\varphi_{m}^{T}\left(\Gamma_{1}^{q}, \ldots, \Gamma_{m}^{q}\right)$ is a purely combinatorial factor (see [Pf1], formulas (3.20) and (3.42)). This is the basic identity which is used for controlling $\Theta_{q}(\Lambda)$. An important property of $\varphi_{m}^{T}\left(\Gamma_{1}^{q}, \ldots, \Gamma_{m}^{q}\right)$ is that $\varphi_{m}^{T}\left(\Gamma_{1}^{q}, \ldots, \Gamma_{m}^{q}\right)$ $=0$ if the following graph is not connected (Lemma 3.3 in [Pf1]): to each $\Gamma_{i}^{q}$ one associates a vertex $v_{i}$, and to each pair $\left\{v_{i}, v_{j}\right\}$ one associates an edge if and only if $\varphi_{2}\left(\Gamma_{i}^{q}, \Gamma_{j}^{q}\right) \neq 0$.

Lemma 3.2. Assume that

$$
C:=\sum_{\Gamma^{q}: \operatorname{supp} \Gamma^{q} \ni 0}\left|\omega\left(\Gamma^{q}\right)\right| \exp \left(\left|\overline{\Gamma^{q}}\right|\right)<\infty .
$$

Then

$$
\sum_{\substack{\Gamma_{1}^{q}: \\ 0 \in \operatorname{supp} \Gamma_{1}^{q}}} \sum_{\Gamma_{2}^{q}} \cdots \sum_{\Gamma_{m}^{q}}\left|\varphi_{m}^{T}\left(\Gamma_{1}^{q}, \ldots, \Gamma_{m}^{q}\right)\right| \prod_{i=1}^{m}\left|\omega\left(\Gamma_{i}^{q}\right)\right| \leq(m-1) ! C^{m} .
$$

If furthermore $C<1$, then (3.10) is true, and the right-hand side of (3.10) is an absolutely convergent sum.

Lemma 3.2 is Lemma 3.5 in [Pf1], where a proof is given. There exists a constant $K_{P}$ such that

$$
\mid\left\{\Gamma^{q}: \operatorname{supp} \Gamma^{q} \ni 0 \text { and }\left|\operatorname{supp} \Gamma^{q}\right|=n\right\} \mid \leq\left(K_{P}\right)^{n} .
$$

If $\omega\left(\Gamma^{q}\right)$ is $\tau$-stable, then there exist $\hat{K}_{0}<\infty$ and $\tau_{0}^{*}<\infty$ so that $\hat{K}_{0} \mathrm{e}^{-\tau_{0}^{*}}<$ 1 , and for all $\tau \geq \tau_{0}^{*}$,

$$
C=\sum_{\Gamma^{q}: \operatorname{supp} \Gamma^{q} \ni 0}\left|\omega\left(\Gamma^{q}\right)\right| \exp \left(\left|\overline{\Gamma^{q}}\right|\right) \leq \sum_{j \geq 1}\left(K_{P}\right)^{j} \mathrm{e}^{-\left(\tau-C_{5}\right) j} \leq \hat{K}_{0} \mathrm{e}^{-\tau} .
$$

If this is true, (3.10) implies ${ }^{29}$ that

\footnotetext{
${ }^{29}$ The corresponding formula (3.58) in [Pf1] is incorrect; a factor $\left|\gamma_{1} \cap \mathbb{Z}_{*}^{2}\right|^{-1}$ is missing.
} 


$$
\beta g_{q}=\sum_{m \geq 1} \frac{1}{m !} \sum_{\substack{\Gamma_{1}^{q} \\ 0 \in \operatorname{supp} \Gamma_{1}^{q}}} \cdots \sum_{\Gamma_{m}^{q}} \frac{1}{\left|\operatorname{supp} \Gamma_{1}^{q}\right|} \varphi_{m}^{T}\left(\Gamma_{1}^{q}, \ldots, \Gamma_{m}^{q}\right) \prod_{i=1}^{m} \omega\left(\Gamma_{i}^{q}\right)
$$

Therefore, there exists $K_{0}<\infty$ so that for all $\tau \geq \tau_{0}^{*}$,

$$
\beta\left|g_{q}\right| \leq \frac{C}{1-C} \leq \frac{\hat{K}_{0}}{1-\hat{K}_{0}} \mathrm{e}^{-\tau} \equiv K_{0} \mathrm{e}^{-\tau} .
$$

One has for all subsets $\Lambda \subset \mathbb{Z}^{d}$

$$
\begin{aligned}
\left|\ln \Theta_{q}(\Lambda)-\beta g_{q}\right| \Lambda|| & \leq \sum_{x \in \partial \Lambda} \sum_{m \geq 1} \frac{1}{m !} \sum_{\substack{\Gamma_{1}^{q}, \ldots, \Gamma_{m}^{q} \\
\exists i \Gamma_{i}^{q} \ni x}}\left|\varphi_{m}^{T}\left(\Gamma_{1}^{q}, \ldots, \Gamma_{m}^{q}\right)\right| \prod_{i=1}^{m}\left|\omega\left(\Gamma_{i}^{q}\right)\right| \\
& \leq K_{0} \mathrm{e}^{-\tau} \partial|\Lambda| .
\end{aligned}
$$

If $\omega\left(\Gamma^{q}\right)=0$ for all $\Gamma^{q}$ such that $\left|\Gamma^{q}\right| \leq m$, then $C \leq \hat{K}_{0}^{m} \mathrm{e}^{-\tau m}$, and

$$
\beta\left|g_{q}\right| \leq\left(K_{0} \mathrm{e}^{-\tau}\right)^{m}
$$

If $n \geq m$ and $m \geq 1$, then

$$
\begin{aligned}
\beta\left|g_{q}^{n}-g_{q}^{m-1}\right| & \leq \sum_{j \geq 1} \frac{1}{j !} \sum_{\substack{\Gamma_{1}^{q} \ni 0, \Gamma_{2}^{q}, \ldots, \Gamma_{j}^{q} \\
\exists i V\left(\Gamma_{i}^{q}\right) \geq m}}\left|\varphi_{j}^{T}\left(\Gamma_{1}^{q}, \ldots, \Gamma_{j}^{q}\right)\right| \prod_{i=1}^{j}\left|\omega_{n}\left(\Gamma_{i}^{q}\right)\right| \\
& \leq \sum_{j \geq 1} \frac{1}{j !} \sum_{\substack{i=1 \\
\Gamma_{1}^{q} \ni 0, \Gamma_{2}^{q}, \ldots, \Gamma_{j}^{q} \\
V\left(\Gamma_{i}^{q}\right) \geq m}}\left|\varphi_{k}^{T}\left(\Gamma_{1}^{q}, \ldots, \Gamma_{j}^{q}\right)\right| \prod_{i=1}^{j}\left|\omega_{n}\left(\Gamma_{i}^{q}\right)\right| \\
& \leq\left(K_{0} \mathrm{e}^{-\tau}\right)^{l(m)} .
\end{aligned}
$$

The last inequality is proved by a straightforward generalization of the proof of Lemma 3.5 in [Pf1]. This proves Lemma 3.1 for $k=0$. The other statements for $k=1,2$ are proved in the same way, by deriving (3.10) term by term. The constant $K_{0}$ is changed into a constant $K_{1}$ or $K_{2}$. $K=\max \left\{K_{0}, K_{1}, K_{2}\right\}$.

\subsection{Construction of the phase diagram in the complex $z$-plane}

At $\beta=\infty$ the phase transition takes place at $\mu=0$, where there is coexistence of the two ground-states. Isakov's approach consists in constructing the phase transition point perturbatively, starting from the point $\mu=0$ 
where there is coexistence of the two ground-states. In an interval $I_{n}$ of $\mu=0$, when $\beta$ is large, but finite, one defines constrained analytic pressures, $p_{1}^{n}$ and $p_{2}^{n}$, for both phases, by taking into account only finitely many different kinds of contours. The (approximate) transition point in the interval $I_{n}$ is given by the value $\mu_{n+1}^{*}$ of $\mu$ such that

$$
p_{1}^{n}\left(\mu_{n+1}^{*}, \beta\right)=p_{2}^{n}\left(\mu_{n+1}^{*}, \beta\right) .
$$

$I_{n+1} \subset I_{n}$, and as $n$ increases, the length of the interval tends to zero. This determines uniquely a point $\mu^{*}$ where all contours are stable. This is the phase coexistence point.

Isakov's analysis is a local analysis around the phase coexistence point, and has been done only for models with two ground-states. It differs from Pirogov-Sinai theory, which is based on the Banach Fixed-Point Theorem, notably because the phase diagram is constructed for the complex variable $z:=\mu+i \nu$, which is essential for studying the singularity of the pressure at $\mu^{*}$. In [Z] another approach is developed, which has similar features with Isakov's approach, and which has been very successful. Zahradník defines, by brute force, i.e. by suppressing unstable contours, truncated pressures for both phases on the whole phase diagram. So, for any value of $\mu$, one has two different truncated pressures, and the equilibrium pressure of the model is equal to the maximal (with my definition of pressure) truncated pressure, so that the transition point is given by the value of $\mu$ for which the two truncated pressures are equal. This approach works well for the complex variable $z$ and for the general situations where there are manifolds with $k$ coexisting phases, with $k \geq 2$ (see [BorIm]). Unfortunately, the truncated pressures are not smooth. It is possible to modify the method and to get $C^{k}$-smooth truncated pressures [BorKo]. However, the truncated pressures cannot be analytic, so they are inappropriate in the present context.

The construction of the phase diagram for the complex variable $z$ is done as follows. It is an iterative procedure. For each integer $n$ one constructs the phase diagram for the constrained pressures $p_{q}^{n}$ (as functions of $z$, see (3.8)). For each $\nu \in \mathbb{R}$ one defines a sequence of intervals

$$
U_{n}(\nu ; \beta):=\left(\mu_{n}^{*}(\nu ; \beta)-b_{n}^{1}, \mu_{n}^{*}(\nu ; \beta)+b_{n}^{2}\right),
$$

with the properties

$$
U_{n}(\nu ; \beta) \subset U_{n-1}(\nu ; \beta),
$$

and

$$
\lim _{n} b_{n}^{q}=0, \quad q=1,2 .
$$

The constrained pressures $p_{q}^{n-1}$ of order $n-1, q=1,2$, are analytic on

$$
\mathbb{U}_{n-1}:=\left\{z \in \mathbb{C}: \operatorname{Re} z \in U_{n-1}(\operatorname{Im} z ; \beta)\right\} .
$$


So, for each interval $U_{n-1}(\nu ; \beta)$ the constrained pressures $p_{q}^{n-1}$ are welldefined and the point $\mu_{n}^{*}(\nu ; \beta)$ is the (unique) solution of the equation

$$
\operatorname{Re}\left(p_{2}^{n-1}\left(\mu_{n}^{*}(\nu ; \beta)+i \nu\right)-p_{1}^{n-1}\left(\mu_{n}^{*}(\nu ; \beta)+i \nu\right)\right)=0 .
$$

The phase coexistence point of the model is given by $\mu^{*}(0 ; \beta)=\lim _{n} \mu_{n}^{*}(0 ; \beta)$. The point $\mu_{n}^{*}(0 ; \beta)$ is also characterized by the following property. Let

$$
p^{n-1}(\mu, \beta):=\max \left\{p_{1}^{n-1}(\mu, \beta), p_{2}^{n-1}(\mu, \beta)\right\} .
$$

Then $\mu_{n}^{*}(0 ; \beta)$ is such that

$$
p^{n-1}(\mu, \beta)= \begin{cases}p_{2}^{n-1}(\mu, \beta) & \text { if } \mu \leq \mu_{n}^{*}(0 ; \beta) \\ p_{1}^{n-1}(\mu, \beta) & \text { if } \mu \geq \mu_{n}^{*}(0 ; \beta) .\end{cases}
$$

Proposition 3.1. Let assumptions $I$ and $I I$ be verified, let $0<\varepsilon<\rho$, and set

$$
U_{0}:=\left(-C_{1}^{-1} \varepsilon, C_{1}^{-1} \varepsilon\right) \quad \text { and } \quad \mathbb{U}_{0}:=\left\{z \in \mathbb{C}: \operatorname{Re} z \in U_{0}\right\} .
$$

Then there exist $\delta=\delta(\beta)$, such that $\lim _{\beta \rightarrow \infty} \delta(\beta)=0$, and $\beta_{0} \in \mathbb{R}^{+}$with the following properties. If $\beta \geq \beta_{0}$, then

$$
\tau(\beta):=\beta(\rho-\varepsilon)-3 C_{0} \delta>0 .
$$

The constants $C_{0}$ and $C_{1}$ are defined in subsection 3.3. Moreover, for $\beta \geq \beta_{0}$,

1. there exists a continuous real-valued function on $\mathbb{R}, \nu \mapsto \mu^{*}(\nu ; \beta) \in$ $U_{0}$, so that $\mu^{*}(\nu ; \beta)+i \nu \in \mathbb{U}_{0}$;

2. if $\mu+i \nu \in \mathbb{U}_{0}$ and $\mu \leq \mu^{*}(\nu ; \beta)$, then the weight $\omega\left(\Gamma^{2}\right)$ is $\tau(\beta)$ stable for all contours $\Gamma^{2}$ with boundary condition $\psi_{2}$, and analytic in $z=\mu+i \nu$ if $\mu<\mu^{*}(\nu ; \beta)$;

3. if $\mu+i \nu \in \mathbb{U}_{0}$ and $\mu \geq \mu^{*}(\nu ; \beta)$, then the weight $\omega\left(\Gamma^{1}\right)$ is $\tau(\beta)$ stable for all contours $\Gamma^{1}$ with boundary condition $\psi_{1}$, and analytic in $z=\mu+i \nu$ if $\mu>\mu^{*}(\nu ; \beta)$.

Corollary 3.1. For $\beta \geq \beta_{0}$ the pressure of the model can be constructed as a real-analytic function $p(\mu, \beta)=g_{2}(\mu, \beta)-\mu h_{1}\left(\psi_{2}\right)$ on $\left\{\mu: \mu<\mu^{*}(0 ; \beta)\right\} \cap$ $U_{0}$. This function has a complex analytic extension in $\{z=\mu+i \nu: \mu<$ $\left.\mu^{*}(\nu ; \beta)\right\} \cap \mathbb{U}_{0}$, which is given by $g_{2}(z, \beta)-z h_{1}\left(\psi_{2}\right)$. Similarly, the pressure can be constructed as a real-analytic function $p(\mu, \beta)=g_{1}(\mu, \beta)-\mu h_{1}\left(\psi_{1}\right)$ on $\left\{\mu: \mu>\mu^{*}(0 ; \beta)\right\} \cap U_{0}$. This function has a complex analytic extension in $\left\{z=\mu+i \nu: \mu>\mu^{*}(\nu ; \beta)\right\} \cap \mathbb{U}_{0}$, which is given by $g_{1}(z, \beta)-z h_{1}\left(\psi_{1}\right)$. At $\mu^{*}(\beta):=\mu^{*}(0 ; \beta)$,

$$
p\left(\mu^{*}(\beta), \beta\right)=g_{2}\left(\mu^{*}(\beta), \beta\right)-\mu^{*}(\beta) h_{1}\left(\psi_{2}\right)=g_{1}\left(\mu^{*}(\beta), \beta\right)-\mu^{*}(\beta) h_{1}\left(\psi_{1}\right) .
$$


Corollary 3.1 is a direct consequence of the convergence of cluster expansion (3.10) and of (3.8).

I first outline the structure of the proof of Proposition 3.1. An intermediate step is to prove a weaker form of stability. One introduces an auxiliary parameter $0<\theta^{\prime}<1$, so that

$$
\rho\left(1-\theta^{\prime}\right)>\varepsilon
$$

The parameter $\theta^{\prime}$ enters into the size of the intervals $U_{n}$, see (3.18); the size of $U_{n}$ is proportional to $\theta^{\prime}$. This parameter controls the volume term of the weight of a contour by the surface energy $\left\|\Gamma^{q}\right\|$ (see (3.16) and (3.17)). By taking $\varepsilon$ smaller, one can choose $\theta^{\prime}$ larger. $\theta^{\star}$ is chosen so that $\theta^{\star}>\theta^{\prime}$ and $\rho\left(1-\theta^{\star}\right)>\varepsilon$. Set

$$
\tau_{\star}(\beta):=\beta\left(\rho\left(1-\theta^{\star}\right)-\varepsilon\right),
$$

and

$$
\delta:=K \mathrm{e}^{-\tau_{\star}(\beta)} \quad(K \text { is the constant in Lemma 3.1). }
$$

Stability of contours is proved inductively as follows. Let $\beta_{0}$ be large enough and assume that $\beta \geq \beta_{0}$ and that for $q=1,2$ the weights $\omega_{n-1}\left(\Gamma^{q}\right)$ are $\tau_{\star}(\beta)$-stable, as well as

$$
\left|\frac{d}{d z} \omega_{n-1}\left(\Gamma^{q}\right)\right| \leq \mathrm{e}^{-\tau_{\star}(\beta)\left|\Gamma^{q}\right|} .
$$

From (3.8) and Lemma 3.1 one obtains

$$
\left|\frac{d}{d z}\left(p_{2}^{n-1}-p_{1}^{n-1}\right)+\Delta\right|=\left|\frac{d}{d z}\left(g_{2}^{n-1}-g_{1}^{n-1}\right)\right| \leq 2 \delta,
$$

and $(m \neq q)$

$$
\begin{aligned}
& \left|\ln \Theta_{q}^{n-1}\left(\operatorname{Int}_{m} \Gamma^{q}\right)-\beta g_{q}^{n-1} V\left(\Gamma^{q}\right)\right|\left|\leq \delta C_{0}\right| \Gamma^{q} \mid \\
& \left|\ln \Theta_{m}^{n-1}\left(\operatorname{Int}_{m} \Gamma^{q}\right)-\beta g_{m}^{n-1} V\left(\Gamma^{q}\right)\right|\left|\leq \delta C_{0}\right| \Gamma^{q} \mid .
\end{aligned}
$$

Let $\Gamma^{q}$ be a contour with $V\left(\Gamma^{q}\right)=n$. Then (always $m \neq q$ )

$$
\begin{aligned}
\left|\omega\left(\Gamma^{q}\right)\right| & =\exp \left[-\beta \operatorname{Re} \mathcal{H}\left(\varphi_{\Gamma^{q}} \mid \psi_{q}\right)\right]\left|\frac{\Theta_{m}\left(\operatorname{Int}_{m} \Gamma^{q}\right)}{\Theta_{q}\left(\operatorname{Int}_{m} \Gamma^{q}\right)}\right| \\
& \leq \exp \left[-\beta\left\|\Gamma^{q}\right\|+\left(\beta \varepsilon+2 C_{0} \delta\right)\left|\Gamma^{q}\right|+\beta \operatorname{Re}\left(p_{m}^{n-1}-p_{q}^{n-1}\right) V\left(\Gamma^{q}\right)\right],
\end{aligned}
$$

because all contours inside $\operatorname{Int}_{m} \Gamma^{q}$ have a volume smaller than $n-1$, and (see $(3.5))$

$$
\left|\operatorname{Re} z a\left(\varphi_{\Gamma^{q}}\right)\right| \leq \varepsilon \quad \forall z \in \mathbb{U}_{0} .
$$

To prove the stability of $\omega\left(\Gamma^{q}\right)$ one must control the volume term in the right-hand side of inequality (3.15). If

$$
\operatorname{Re}\left(p_{1}^{n-1}-p_{2}^{n-1}\right) V\left(\Gamma^{2}\right) \leq \theta^{\prime}\left\|\Gamma^{2}\right\|
$$


and

$$
\operatorname{Re}\left(p_{2}^{n-1}-p_{1}^{n-1}\right) V\left(\Gamma^{1}\right) \leq \theta^{\prime}\left\|\Gamma^{1}\right\|,
$$

then $\omega\left(\Gamma^{2}\right)$ and $\omega\left(\Gamma^{1}\right)$ are $\tau_{\star}(\beta)$-stable. Indeed, these inequalities imply

$$
\begin{aligned}
\left|\omega\left(\Gamma^{q}\right)\right| & \leq \exp \left[-\beta\left(1-\theta^{\prime}\right)\left\|\Gamma^{q}\right\|+\left(\beta \varepsilon+2 C_{0} \delta\right)\left|\Gamma^{q}\right|\right] \\
& \leq \exp \left[-\beta\left(\left(1-\theta^{\star}\right) \rho-\varepsilon\right)\left|\Gamma^{q}\right|\right] .
\end{aligned}
$$

The last inequality is always true by choosing $\beta_{0}$ large enough (independently of $\left.\Gamma^{q}\right)$. Verification of the inequalities (3.16) and (3.17) is possible because (3.14) provides a sharp estimate of the derivative of $p_{2}^{n-1}-p_{1}^{n-1}$. Proof of Proposition 3.1. Let $\theta^{\prime}$ be chosen as above, and $b_{0}:=\varepsilon C_{1}^{-1}$. $p_{q}^{0}(\mu+i \nu)$ is defined on the interval $U_{0}(\nu ; \beta):=\left(-b_{0}, b_{0}\right)$, and set $\mu_{0}^{*}(\nu ; \beta):=$ 0 . The two decreasing sequences $\left\{b_{n}^{q}\right\}, q=1,2$ and $n \geq 1$, are chosen as (see $(3.23))$

$$
b_{n}^{1} \equiv b_{n}^{2}:=\frac{\chi \theta^{\prime}}{(\Delta+2 \delta) n^{\frac{1}{d}}}, n \geq 1 .
$$

The constant $\chi$ is the best constant such that

$$
V\left(\Gamma^{q}\right)^{\frac{d-1}{d}} \leq \chi^{-1}\left\|\Gamma^{q}\right\| \quad \forall \Gamma^{q}, q=1,2 .
$$

Taking $\beta$ large enough, we may assume that

$$
b_{n}^{q}-b_{n+1}^{q}>\frac{2 \delta^{l(n)}}{\beta(\Delta-2 \delta)}, \quad \forall n \geq 1 .
$$

On $\mathbb{U}_{0}$ all contours $\Gamma$ with volume zero are $\beta(\rho-\varepsilon)$-stable, and, if $\beta_{0}$ is large enough,

$$
\left|\frac{d}{d z} \omega(\Gamma)\right| \leq \beta C_{1}|\Gamma| \mathrm{e}^{-\beta(\rho-\varepsilon)|\Gamma|} \leq \beta C_{1} \mathrm{e}^{-[\beta(\rho-\varepsilon)-1]|\Gamma|} \leq \mathrm{e}^{-\tau_{\star}(\beta)|\Gamma|} .
$$

The proof of Proposition 3.1 consists in proving iteratively the following four statements.

A. There exists a unique continuous solution $\nu \mapsto \mu_{n}^{*}(\nu ; \beta)$ of the equation

$$
\operatorname{Re}\left(p_{2}^{n-1}\left(\mu_{n}^{*}(\nu ; \beta)+i \nu\right)-p_{1}^{n-1}\left(\mu_{n}^{*}(\nu ; \beta)+i \nu\right)\right)=0,
$$

so that (3.12) holds.

B. For any contour $\Gamma^{q}, \omega_{n}\left(\Gamma^{q}\right)$ is well-defined and analytic on $\mathbb{U}_{n}$, and $\omega_{n}\left(\Gamma^{q}\right)$ is $\tau_{\star}(\beta)$-stable. Moreover, $\Theta_{q}^{n}(\Lambda) \neq 0$ for any finite $\Lambda$, and $p_{q}^{n}(z ; \beta)$ is analytic on $\mathbb{U}_{n}$.

C. On $\mathbb{U}_{n},\left|\frac{d}{d z} \omega_{n}\left(\Gamma^{q}\right)\right| \leq \mathrm{e}^{-\tau_{\star}(\beta)\left|\Gamma^{q}\right|}$. 
D. If $z=\mu+i \nu \in \mathbb{U}_{0}$ and $\mu \leq \mu_{n}^{*}(\nu ; \beta)-b_{n}^{1}$, then $\omega\left(\Gamma^{2}\right)$ is $\tau(\beta)$ stable for any $\Gamma^{2}$ with boundary condition $\psi_{2}$. If $z=\mu+i \nu \in \mathbb{U}_{0}$ and $\mu \geq \mu_{n}^{*}(\nu ; \beta)+b_{n}^{2}$, then $\omega\left(\Gamma^{1}\right)$ is $\tau(\beta)$-stable for any $\Gamma^{1}$ with boundary condition $\psi_{1}$.

Notice that A, B and C are sufficient to prove the existence of a point with two stable coexisting phases.

Assume that the construction has been done for all $k \leq n-1$.

A. Proof of the existence of $\mu_{n}^{*}(\nu ; \beta) \in \mathbb{U}_{n-1}$.

$\mu_{n}^{*}(\nu ; \beta)$ is solution of the equation

$$
\operatorname{Re}\left(p_{2}^{n-1}\left(\mu_{n}^{*}(\nu ; \beta)+i \nu\right)-p_{1}^{n-1}\left(\mu_{n}^{*}(\nu ; \beta)+i \nu\right)\right)=0 .
$$

The value of $\nu$ is fixed, and set

$$
F^{k}(\mu):=p_{2}^{k}(\mu+i \nu)-p_{1}^{k}(\mu+i \nu) .
$$

One proves that

$$
\mu \mapsto \operatorname{Re} F^{n-1}(\mu)
$$

is strictly decreasing, and takes positive and negative values. If $\mu^{\prime}+i \nu \in$ $\mathbb{U}_{n-1}$, then

$$
\begin{aligned}
F^{n-1}\left(\mu^{\prime}\right) & =F^{n-1}\left(\mu^{\prime}\right)-F^{n-2}\left(\mu_{n-1}^{*}\right) \\
& =F^{n-1}\left(\mu^{\prime}\right)-F^{n-1}\left(\mu_{n-1}^{*}\right)+F^{n-1}\left(\mu_{n-1}^{*}\right)-F^{n-2}\left(\mu_{n-1}^{*}\right) \\
& =\int_{\mu_{n-1}^{*}}^{\mu^{\prime}} \frac{d}{d \mu} F^{n-1}(\mu) d \mu+\left(g_{2}^{n-1}-g_{2}^{n-2}\right)\left(\mu_{n-1}^{*}+i \nu\right) \\
& -\left(g_{1}^{n-1}-g_{1}^{n-2}\right)\left(\mu_{n-1}^{*}+i \nu\right) .
\end{aligned}
$$

If $V(\Gamma)=n-1$, then $|\Gamma| \geq l(n-1)$. Therefore, by Lemma 3.1,

$$
\left|\left(g_{q}^{n-1}-g_{q}^{n-2}\right)\left(\mu_{n-1}^{*}+i \nu\right)\right| \leq \beta^{-1} \delta^{l(n-1)} .
$$

If $z^{\prime}=\mu^{\prime}+i \nu \in \mathbb{U}_{n-1}$, then (3.21), (3.14) and (3.22) imply

$$
-\Delta\left(\mu^{\prime}-\mu_{n-1}^{*}\right)-2 \delta\left|\mu^{\prime}-\mu_{n-1}^{*}\right|-2 \beta^{-1} \delta^{l(n-1)} \leq \operatorname{Re} F^{n-1}\left(z^{\prime}\right)
$$

and

$$
\operatorname{Re} F^{n-1}\left(z^{\prime}\right) \leq-\Delta\left(\mu^{\prime}-\mu_{n-1}^{*}\right)+2 \delta\left|\mu^{\prime}-\mu_{n-1}^{*}\right|+2 \beta^{-1} \delta^{l(n-1)} .
$$

Since (3.20) holds,

$$
b_{n-1}^{q}>b_{n-1}^{q}-b_{n}^{q}>\frac{2 \delta^{l(n-1)}}{\beta(\Delta-2 \delta)},
$$


so that $\operatorname{Re} F^{n-1}\left(\mu_{n-1}^{*}-b_{n-1}^{1}\right)>0$ and $\operatorname{Re} F^{n-1}\left(\mu_{n-1}^{*}+b_{n-1}^{2}\right)<0$. Since $\mu \mapsto \operatorname{Re} F^{n-1}(\mu)$ is strictly decreasing (see (3.14)), existence and uniqueness of $\mu_{n}^{*}$ is proved. Moreover, choosing $\mu^{\prime}=\mu_{n}^{*}(\nu ; \beta)$ in (3.21), one gets

$$
\left|\mu_{n}^{*}(\nu ; \beta)-\mu_{n-1}^{*}(\nu ; \beta)\right| \leq \frac{2 \delta^{l(n-1)}}{\beta(\Delta-2 \delta)} .
$$

Therefore $\mathbb{U}_{n} \subset \mathbb{U}_{n-1}$. The Implicit Function Theorem implies that $\nu \mapsto$ $\mu_{n}^{*}(\nu ; \beta)$ is continuous.

B. Proof of the $\tau_{\star}$-stability on $\mathbb{U}_{n}$ of the weights $\omega_{n}\left(\Gamma^{q}\right)$ of all contours $\Gamma^{q}$, $q=1,2$.

By the induction hypothesis the weights $\omega_{n}\left(\Gamma^{q}\right)$ are analytic in $\mathbb{U}_{n-1}$. This implies that $p_{q}^{n}$ is analytic on $\mathbb{U}_{n}$. The proof of the stability has been already outlined. Let $\Gamma^{q}$ be a contour with $V\left(\Gamma^{q}\right)=n$. One verifies (3.16) if $\mu \leq \mu_{n}^{*}+b_{n}^{2}$, respectively (3.17) if $\mu \geq \mu_{n}^{*}-b_{n}^{1}$. The choice of $\left\{b_{n}^{q}\right\}$ and the isoperimetric inequality (3.19) imply

$$
\begin{aligned}
\left|\operatorname{Re}\left(p_{m}^{n-1}-p_{q}^{n-1}\right)\right| \frac{V\left(\Gamma^{q}\right)}{\left\|\Gamma^{q}\right\|} & =\left|\operatorname{Re} \int_{\mu_{n}^{*}}^{\mu} \frac{d}{d \mu}\left(p_{m}^{n-1}-p_{q}^{n-1}\right) d \mu\right| \frac{V\left(\Gamma^{q}\right)}{\left\|\Gamma^{q}\right\|} \\
& \leq\left|\mu-\mu_{n}^{*}\right|(\Delta+2 \delta) \frac{V\left(\Gamma^{q}\right)}{\left\|\Gamma^{q}\right\|} \\
& \leq b_{n}(\Delta+2 \delta) V\left(\Gamma^{q}\right)^{\frac{1}{d}} \chi^{-1} \\
& \leq \theta^{\prime}
\end{aligned}
$$

Since the cluster expansion of $\ln \Theta_{q}^{n}(\Lambda)$ is absolutely convergent, $\Theta_{q}^{n}(\Lambda) \neq 0$ and $p_{q}^{n}$ is analytic on $\mathbb{U}_{n}$.

C. Proof of the $\tau_{\star}$-stability of $\frac{d}{d z} \omega_{n}\left(\Gamma^{q}\right)$ on $\mathbb{U}_{n}$.

Let $V\left(\Gamma^{q}\right)=n$; from $(3.4)$

$$
\begin{aligned}
\frac{d}{d z} \omega_{n}\left(\Gamma^{q}\right)= & \omega_{n}\left(\Gamma^{q}\right)\left(-\beta a\left(\varphi_{\Gamma^{q}}\right)-\beta\left(h_{1}\left(\psi_{m}\right)-h_{1}\left(\psi_{q}\right)\right) V\left(\Gamma^{q}\right)\right. \\
& \left.+\frac{d}{d z}\left(\ln \Theta_{m}\left(\operatorname{Int}_{m} \Gamma^{q}\right)-\ln \Theta_{q}\left(\operatorname{Int}_{m} \Gamma^{q}\right)\right)\right) .
\end{aligned}
$$

There exists a constant ${ }^{30} C_{3}$ such that

$$
\begin{aligned}
\left|\frac{d}{d z} \omega_{n}\left(\Gamma^{q}\right)\right| & \leq \beta\left|\omega_{n}\left(\Gamma^{q}\right)\right|\left(\left|\Gamma^{q}\right|\left(C_{1}+2 \delta C_{0}\right)+V\left(\Gamma^{q}\right)(\Delta+2 \delta)\right) \\
& \leq \beta C_{3}\left|\omega_{n}\left(\Gamma^{q}\right)\right|\left|\Gamma^{q}\right|^{\frac{d}{d-1}} \\
& \leq \mathrm{e}^{-\tau_{\star}(\beta)\left|\Gamma^{q}\right|}
\end{aligned}
$$

provided that $\beta_{0}$ is large enough (use (3.15) for controlling $\left|\omega_{n}\left(\Gamma^{q}\right)\right|$ ).

${ }^{30}$ See (3.5), (3.19) and (3.6); for $C_{0}$, see footnote 20. 
D. Proof of the $\tau(\beta)$-stability of any contour $\Gamma^{2}$ with boundary condition $\psi_{2}$, if $\mu \leq \mu_{n}^{*}(\nu ; \beta)-b_{n}^{1}$.

Using the induction hypothesis it is sufficient to prove this statement for $z=\mu+i \nu \in \mathbb{U}_{n-1}$ and $\mu \leq \mu_{n}^{*}(\nu ; \beta)-b_{n}^{1}$.

If $z=\mu+i \nu \in \mathbb{U}_{n-1}$, then all contours with volume smaller than $n-1$ are $\tau_{\star}(\beta)$-stable; $(3.14)$ and $\mu \leq \mu_{n}^{*}$ imply that $\mu \mapsto \operatorname{Re}\left(p_{2}^{n-1}-p_{1}^{n-1}\right)(\mu+i \nu)$ is strictly decreasing. If $\mu \leq \mu_{n}^{*}(\nu ; \beta)-b_{n}^{1}$, then (see (3.18) and (3.20))

$$
\begin{aligned}
\beta \operatorname{Re}\left(p_{2}^{n-1}-p_{1}^{n-1}\right)(\mu+i \nu) & =-\beta \int_{\mu}^{\mu_{n}^{*}} \frac{d}{d \mu} \operatorname{Re}\left(p_{2}^{n-1}-p_{1}^{n-1}\right)(\mu+i \nu) d \mu \\
& \geq-\beta \int_{\mu_{n}^{*}-b_{n}^{1}}^{\mu_{n}^{*}} \frac{d}{d \mu} \operatorname{Re}\left(p_{2}^{n-1}-p_{1}^{n-1}\right)(\mu+i \nu) d \mu \\
& \geq \beta b_{n}^{1}(\Delta-2 \delta) \geq 2 \delta^{l(n)}
\end{aligned}
$$

Suppose that $V\left(\Gamma^{2}\right) \leq n$. From (3.24) and (3.15) it follows that $\omega\left(\Gamma^{2}\right)$ is $\tau(\beta)$-stable. Moreover, if $|\Lambda| \leq n$, then

$$
\left|\exp \left[-\beta z\left(h_{1}\left(\psi_{1}\right)-h_{1}\left(\psi_{2}\right)\right)|\Lambda|\right] \frac{\Theta_{1}(\Lambda)}{\Theta_{2}(\Lambda)}\right| \leq \mathrm{e}^{3 \delta \partial|\Lambda|} .
$$

Indeed, all contours inside $\Lambda$ are $\tau_{\star}(\beta)$-stable. By Lemma 3.1 and (3.24),

$$
\begin{aligned}
\left|\mathrm{e}^{-\beta z\left(h_{1}\left(\psi_{1}\right)-h_{1}\left(\psi_{2}\right)\right)|\Lambda|} \frac{\Theta_{1}(\Lambda)}{\Theta_{2}(\Lambda)}\right| & \leq\left|\mathrm{e}^{-\beta\left(z h_{1}\left(\psi_{1}\right)-z h_{1}\left(\psi_{2}\right)-g_{1}^{n-1}+g_{2}^{n-1}\right)|\Lambda|}\right| \mathrm{e}^{2 \delta \partial|\Lambda|} \\
& =\mathrm{e}^{-\beta \operatorname{Re}\left(p_{2}^{n-1}(z)-p_{1}^{n-1}(z)\right)|\Lambda|} \mathrm{e}^{2 \delta \partial|\Lambda|} \\
& \leq \mathrm{e}^{2 \delta \partial|\Lambda|} .
\end{aligned}
$$

Suppose that (3.25) is true for any $\Lambda$ and set $\Lambda:=\operatorname{Int}_{1} \Gamma^{2}$. From the definition of $\omega\left(\Gamma^{2}\right)$ and from (3.4) it follows that $\omega\left(\Gamma^{2}\right)$ is $\tau(\beta)$-stable.

The argument to prove (3.25) for any $\Lambda$ is due to Zahradnik [Z]. One knows from above that if $V\left(\Gamma^{2}\right) \leq n$, then the contour is $\tau(\beta)$-stable. Let $k>n$ and $|\Lambda|=k$. One proves (3.25) by induction on $k$. The induction hypothesis implies that $\omega\left(\Gamma^{2}\right)(z)$ is $\tau(\beta)$-stable if $V\left(\Gamma^{2}\right) \leq k$. On the other hand, one controls only the weights of the contours $\Gamma_{j}^{1}$ which have a volume smaller than $n-1$. These contours are $\tau_{\star}$-stable. A contour $\Gamma_{j}^{1}$ which has a volume larger than $n$ is called large. The first step is to rewrite $\Theta_{1}(\Lambda)$ by re-summing over small contours. The partition function $\Theta_{1}(\Lambda)$ is given by (see (3.1))

$$
\Theta_{1}(\Lambda)=\sum \prod_{j=1}^{r} \Theta\left(\Gamma_{j}^{1}\right)
$$

where the sum is over all families $\left\{\Gamma_{1}^{1}, \ldots, \Gamma_{r}^{1}\right\}$ of compatible external contours in $\Lambda$. Suppose that the contours $\Gamma_{1}^{1}, \ldots \Gamma_{p}^{1}$ are large and all other 
contours $\Gamma_{p+1}^{1}, \ldots \Gamma_{r}^{1}$ not large. Set

$$
\operatorname{Ext}_{1}^{p}(\Lambda):=\left(\bigcap_{j=1}^{p} \operatorname{Ext}_{j}^{1}\right) \cap \Lambda
$$

Summing over all contours which are not large, $\Theta_{1}(\Lambda)$ can be written as (see $(3.2))$

$$
\sum \Theta_{1}^{n-1}\left(\operatorname{Ext}_{1}^{p}(\Lambda)\right) \prod_{j=1}^{p} \exp \left[-\beta \mathcal{H}\left(\varphi_{\Gamma_{j}^{1}} \mid \psi_{1}\right)\right] \Theta_{1}\left(\operatorname{Int}_{1} \Gamma_{j}^{1}\right) \Theta_{2}\left(\operatorname{Int}_{2} \Gamma_{j}^{1}\right) ;
$$

the sums are over all families $\left\{\Gamma_{1}^{1}, \ldots, \Gamma_{p}^{1}\right\}$ of compatible external large contours in $\Lambda$. By Lemma 3.1 one controls $\Theta_{1}^{n-1}\left(\operatorname{Ext}_{1}^{p}(\Lambda)\right), \Theta_{2}\left(\operatorname{Int}_{2} \Gamma_{j}^{1}\right)$ and $\Theta_{2}(\Lambda)$. To control $\Theta_{1}\left(\operatorname{Int}_{1} \Gamma_{j}^{1}\right)$ one writes

$$
\Theta_{1}\left(\operatorname{Int}_{1} \Gamma_{j}^{1}\right)=\frac{\Theta_{1}\left(\operatorname{Int}_{1} \Gamma_{j}^{1}\right)}{\Theta_{2}\left(\operatorname{Int}_{1} \Gamma_{j}^{1}\right)} \Theta_{2}\left(\operatorname{Int}_{1} \Gamma_{j}^{1}\right) .
$$

The quotient $\Theta_{1}\left(\operatorname{Int}_{1} \Gamma_{j}^{1}\right) \Theta_{2}\left(\operatorname{Int}_{2} \Gamma_{j}^{1}\right)^{-1}$ is controlled by the induction hypothesis and $\Theta_{2}\left(\operatorname{Int}_{1} \Gamma_{j}^{1}\right)$ by Lemma 3.1. Taking into account $\operatorname{Int} \Gamma_{j}^{1}=$ $\operatorname{Int}_{1} \Gamma_{j}^{1} \cup \operatorname{Int}_{2} \Gamma_{j}^{1}$ and

$$
\partial\left|\operatorname{Ext}_{1}^{p}(\Lambda)\right| \leq \partial|\Lambda|+\sum_{j=1}^{p} C_{0}\left|\Gamma_{j}^{1}\right|
$$

one gets

$$
\begin{aligned}
\left|\Theta_{1}(\Lambda)\right| \leq & \mathrm{e}^{\delta \partial|\Lambda|} \sum \mathrm{e}^{\beta \operatorname{Re} g_{1}^{n-1}\left|\operatorname{Ext}_{1}^{p}(\Lambda)\right|} \prod_{j=1}^{p} \mathrm{e}^{-\left(\beta \rho-\beta \varepsilon-5 C_{0} \delta\right)\left|\Gamma_{j}^{1}\right|} \\
& \cdot \prod_{j=1}^{p} \mathrm{e}^{\beta\left(\mu h_{1}\left(\psi_{1}\right)-\mu h_{1}\left(\psi_{2}\right)+\operatorname{Re} g_{2}^{k}\right)\left|\operatorname{Int}_{j}^{1}\right|} .
\end{aligned}
$$

One has

$$
|\Lambda|=\left|\operatorname{Ext}_{1}^{p}(\Lambda)\right|+\sum_{j=1}^{p}\left|\Gamma_{j}^{1}\right|+\sum_{j=1}^{p}\left|\operatorname{Int} \Gamma_{j}^{1}\right| .
$$

Since $\beta\left|\operatorname{Re} g_{1}^{n-1}\right| \leq \delta$, by adding and subtracting $\sum_{j} \operatorname{Re} g_{1}^{n-1}\left|\Gamma_{j}^{1}\right|$, one gets

$$
\begin{gathered}
\left|\mathrm{e}^{-\beta z\left(h_{1}\left(\psi_{1}\right)-h_{1}\left(\psi_{2}\right)\right)|\Lambda|} \frac{\Theta_{1}(\Lambda)}{\Theta_{2}(\Lambda)}\right| \leq \mathrm{e}^{2 \delta \partial|\Lambda|} \sum \mathrm{e}^{-\beta \operatorname{Re}\left(p_{2}^{k}-p_{1}^{n-1}\right)\left|\operatorname{Ext}_{1}^{p}(\Lambda)\right|} \\
\cdot \prod_{j=1}^{p} \mathrm{e}^{-\left(\beta \rho-\beta \varepsilon-6 C_{0} \delta\right)\left|\Gamma_{j}^{1}\right|} \mathrm{e}^{-\beta \operatorname{Re}\left(p_{2}^{k}-p_{1}^{n-1}\right)\left|\Gamma_{j}^{1}\right|}
\end{gathered}
$$


Let

$$
\hat{\tau}(\beta):=\beta(\rho-\varepsilon)-6 C_{0} \delta .
$$

From (3.24) and Lemma 3.1 one has

$$
\begin{aligned}
\beta \operatorname{Re}\left(p_{2}^{k}-p_{1}^{n-1}\right) & =\beta \operatorname{Re}\left(p_{2}^{n-1}-p_{1}^{n-1}\right)+\beta \operatorname{Re}\left(p_{2}^{k}-p_{2}^{n-1}\right) \\
& =\beta \operatorname{Re}\left(p_{2}^{n-1}-p_{1}^{n-1}\right)+\beta \operatorname{Re}\left(g_{2}^{k}-g_{2}^{n-1}\right) \geq \delta^{l(n)} .
\end{aligned}
$$

Hence,

$$
\begin{aligned}
\left|\mathrm{e}^{-\beta z\left(h_{1}\left(\psi_{1}\right)-h_{1}\left(\psi_{2}\right)\right)|\Lambda|} \frac{\Theta_{1}(\Lambda)}{\Theta_{2}(\Lambda)}\right| \leq \mathrm{e}^{2 \delta \partial|\Lambda|} \sum \mathrm{e}^{-\delta^{l(n)}\left|\operatorname{Ext}_{1}^{p}(\Lambda)\right|} & \\
& \cdot \prod_{j=1}^{p} \mathrm{e}^{-\left(\delta^{l(n)}+\hat{\tau}(\beta)\right)\left|\Gamma_{j}^{1}\right|} .
\end{aligned}
$$

One then defines ${ }^{31}$

$$
\hat{\omega}(\Gamma):= \begin{cases}\mathrm{e}^{-\left(\hat{\tau}(\beta)-C_{0} \delta\right)|\Gamma|} & \text { if }|\Gamma| \geq l(n) ; \\ 0 & \text { otherwise. }\end{cases}
$$

Let $\hat{\Theta}(\Lambda)$ be defined by $(3.3)$, replacing $\omega\left(\Gamma^{q}\right)$ by $\hat{\omega}(\Gamma)$, and let

$$
\hat{g}:=\lim _{\Lambda \uparrow \mathbb{Z}^{d}} \frac{1}{\beta|\Lambda|} \log \hat{\Theta}(\Lambda) .
$$

We can take $\beta_{0}$ large enough so that Lemma 3.1 applies for all $\beta \geq \beta_{0}$, and so that $\beta|\hat{g}| \leq \delta^{l(n)}$, and for any $\Lambda$

$$
|\ln \hat{\Theta}(\Lambda)-\beta \hat{g}| \Lambda|| \leq \delta \partial|\Lambda| \text {. }
$$

Therefore

$$
\begin{gathered}
\left|\mathrm{e}^{-\beta z\left(h_{1}\left(\psi_{1}\right)-h_{1}\left(\psi_{2}\right)\right)|\Lambda|} \frac{\Theta_{1}(\Lambda)}{\Theta_{2}(\Lambda)}\right| \leq \mathrm{e}^{2 \delta \partial|\Lambda|-\beta \hat{g}|\Lambda|} \sum \prod_{j=1}^{p} \mathrm{e}^{-\hat{\tau}(\beta)\left|\Gamma_{j}^{1}\right|} \mathrm{e}^{\beta \hat{g}\left|\operatorname{Int} \Gamma_{j}^{1}\right|} \\
\leq \mathrm{e}^{2 \delta \partial|\Lambda|-\beta \hat{g}|\Lambda|} \sum \prod_{j=1}^{p} \mathrm{e}^{-\left(\hat{\tau}(\beta)-C_{0} \delta\right)\left|\Gamma_{j}^{1}\right|} \hat{\Theta}\left(\operatorname{Int} \Gamma_{j}^{1}\right) .
\end{gathered}
$$

In the above formula $\mathrm{e}^{\beta \hat{g}\left|\operatorname{Int} \Gamma^{1}\right|}$ is estimated by $\hat{\Theta}\left(\operatorname{Int} \Gamma^{1}\right) \mathrm{e}^{C_{0} \delta\left|\Gamma^{1}\right|}$. Summing over external contours one gets

$$
\left|\mathrm{e}^{-\beta z\left(h_{1}\left(\psi_{1}\right)-h_{1}\left(\psi_{2}\right)\right)|\Lambda|} \frac{\Theta_{1}(\Lambda)}{\Theta_{2}(\Lambda)}\right| \leq \mathrm{e}^{2 \delta \partial|\Lambda|-\beta \hat{g}|\Lambda|} \hat{\Theta}(\Lambda) \leq \mathrm{e}^{3 \delta \partial|\Lambda|} .
$$

${ }^{31}$ The term $C_{0} \delta$ is introduced for controlling boundary terms later on. 
It is not difficult to prove more regularity for the curve $\nu \mapsto \mu^{*}(\nu ; \beta)$. But this is not necessary for these lectures. If $\beta$ is sufficiently large, then for all $n \geq 1$

$$
\frac{d}{d \nu} \mu_{n}^{*}(0 ; \beta)=0
$$

and

$$
\left|\frac{d^{2}}{d \nu^{2}} \mu_{n}^{*}(\nu ; \beta)\right| \leq \frac{2 \delta}{\Delta-2 \delta}\left(\left(\frac{2 \delta}{\Delta-2 \delta}\right)^{2}+\frac{2 \delta}{\Delta-2 \delta}+1\right) .
$$

The first formula is a consequence of the reality of the constrained pressures on the real axis, which implies that $\nu \mapsto \mu_{n}^{*}(\nu ; \beta)$ is an even function. The next proposition gives an estimate of $\left|\mu^{*}(\nu ; \beta)-\mu_{n}^{*}(\nu ; \beta)\right|$ and improved estimates for the derivative of the weight of a contour (see [FrPf1]).

Proposition 3.2. Under the conditions of Proposition 3.1, there exist $\beta_{0} \in$ $\mathbb{R}^{+}$and a constant $D$ so that the following holds for all $\beta \geq \beta_{0}$. Let

$$
\tau^{\prime}(\beta):=\tau(\beta)-D
$$

1. If $\mu+i \nu \in \mathbb{U}_{0}$ and $\mu \leq \mu^{*}(\nu ; \beta)$, then

$$
\left|\frac{d}{d z} \omega\left(\Gamma^{2}\right)(z)\right| \leq \beta C_{3} \mathrm{e}^{-\tau^{\prime}(\beta)\left|\Gamma^{2}\right|} .
$$

2. If $\mu+i \nu \in \mathbb{U}_{0}$ and $\mu \geq \mu^{*}(\nu ; \beta)$, then

$$
\left|\frac{d}{d z} \omega\left(\Gamma^{1}\right)(z)\right| \leq \beta C_{3} \mathrm{e}^{-\tau^{\prime}(\beta)\left|\Gamma^{1}\right|} .
$$

3. Moreover

$$
\left|\mu^{*}(\nu ; \beta)-\mu_{n}^{*}(\nu ; \beta)\right| \leq \frac{2 \delta^{l(n)}}{\beta(\Delta-2 \delta)}
$$

\section{Absence of analytic continuation for lattice models with short-range interactions}

The setting is the same as in subsection 3.1. At large $\beta$ the model with hamiltonian $\mathcal{H}^{\mu}=\mathcal{H}_{0}+\mu \mathcal{H}^{1}$ has a first order phase transition at

$$
\mu^{*} \equiv \mu^{*}(\beta):=\mu^{*}(0, \beta),
$$

with two coexisting phases. For $\mu<\mu^{*}(\beta)$, respectively $\mu>\mu^{*}(\beta)$, the pressure $p(\mu, \beta)$ is real-analytic in $\mu$.

In the complex $z$-plane, there is a line of "transition points", which is defined by the property that on this line all contours are stable; it is given by

$$
\operatorname{Re} p_{2}\left(z^{*}\right)=\operatorname{Re} p_{1}\left(z^{*}\right)
$$


Since $z^{*} \equiv \mu^{*}(\nu ; \beta)+i \nu$,

$$
\operatorname{Re} g_{1}\left(z^{*}\right)-\mu^{*}(\nu ; \beta) h_{1}\left(\psi_{1}\right)=\operatorname{Re} g_{2}\left(z^{*}\right)-\mu^{*}(\nu ; \beta) h_{1}\left(\psi_{2}\right) .
$$

With $\delta$ as in the proof of Proposition 3.1, one has (see (3.28)), for any real $\nu$,

$$
\left|\mu^{*}(\nu ; \beta)\right| \leq \frac{2 \delta}{\beta \Delta} .
$$

Theorem 4.1. If the assumptions $I$ and II are satisfied, then there exist an open interval $\left(-\varepsilon^{\prime}, \varepsilon^{\prime}\right)$ and $\beta^{*}<\infty$ such that for any $\beta \geq \beta^{*}$

1. the pressure has a $C^{\infty}$ continuation in $\left(-\varepsilon^{\prime}, \mu^{*}(\beta)\right]$;

2. the pressure has a $C^{\infty}$ continuation in $\left[\mu^{*}(\beta), \varepsilon^{\prime}\right)$;

3. there is no analytic continuation of the pressure from $\mu<\mu^{*}(\beta)$ to $\mu>\mu^{*}(\beta)$ across $\mu^{*}(\beta)$, or vice-versa.

Theorem 4.1 was proved in 1984 by Isakov for the Ising model [I1], and then extended in the case of models with two ground-states verifying Peierls' condition, and under additional assumptions [I2]. The only assumptions here are that Peierls' condition holds and that $\mathcal{H}_{1}$ splits the degeneracy of the ground-states of $\mathcal{H}_{0}$. The proof given in [FrPf1] follows in essence that of Isakov in [I1].

Since the pressure has $C^{\infty}$ continuations up to $\mu^{*}$, one can write two Taylor series for the pressure at $\mu^{*}$. The absence of an analytic extension at $\mu^{*}$ is a consequence of estimates of the derivatives of the pressure of the kind

$$
\left|\frac{d^{k_{n}}}{d \mu^{k_{n}}} p\left(\mu^{*} \pm, \beta\right)\right| \sim\left(k_{n} !\right)^{\frac{d}{d-1}}
$$

for a divergent sequence $\left\{k_{n}\right\}$.

\subsection{Outline of the proof of Theorem 4.1}

The inverse temperature $\beta$ is sufficiently large, but fixed. I have divided the proof into seven steps.

I. Although the statements of Theorem 4.1 concern the thermodynamic limit, most proofs are done by considering finite-volume partition functions, in order to exploit the analyticity of the weights of contours in the neighbourhood of the transition point $\mu^{*}$. Only at the very end of the proof (step VII) one takes the thermodynamic limit. Isakov represents the partition function $\Theta_{q}(\Lambda)$ as a finite product of objects, indexed by the contours in $\Lambda$, so that

$$
g_{\Lambda}^{q}:=\frac{1}{\beta|\Lambda|} \ln \Theta_{q}(\Lambda)=-\frac{1}{\beta|\Lambda|} \sum_{\Gamma^{q} \subset \Lambda} u_{\Lambda}\left(\Gamma^{q}\right),
$$


is a sum with finitely many terms. Let $\Lambda=\Lambda(L)$ be the cubic box

$$
\Lambda(L):=\left\{x \in \mathbb{Z}^{d}:|x| \leq L\right\} .
$$

One introduces a linear order, denoted by $\leq$, among the finite set of all contours $\Gamma^{q} \subset \Lambda$ with boundary condition $\psi_{q}$. The linear order is such that $V\left(\Gamma^{\prime q}\right) \leq V\left(\Gamma^{q}\right)$ if $\Gamma^{\prime q} \leq \Gamma^{q}$. One chooses an enumeration of the contours, $\Gamma_{1}^{q}, \Gamma_{2}^{q}, \ldots$, and denote the predecessor of $\Gamma^{q}$ in that enumeration by $i\left(\Gamma^{q}\right)$ (if $\Gamma^{q}$ is not the smallest contour). Then, one introduces for each $\Gamma^{q}$ a partition function $\Theta_{\Gamma^{q}}(\Lambda)$,

$$
\Theta_{\Gamma^{q}}(\Lambda):=1+\sum \prod_{i=1}^{n} \omega\left(\Gamma_{i}^{q^{\prime}}\right)
$$

where the sum is over all families of compatible contours $\left\{\Gamma_{1}^{q \prime}, \ldots, \Gamma_{n}^{q^{\prime}}\right\}$ with the property that $\Gamma_{j}^{q \prime} \leq \Gamma^{q}$ for all $j$. The partition function $\Theta_{q}(\Lambda)$ is written as

$$
\Theta_{q}(\Lambda)=\prod_{\Gamma^{q} \subset \Lambda} \frac{\Theta_{\Gamma^{q}}(\Lambda)}{\Theta_{i\left(\Gamma^{q}\right)}(\Lambda)} .
$$

By convention $\Theta_{i\left(\Gamma^{q}\right)}(\Lambda):=1$ when $\Gamma^{q}$ is the smallest contour. Let

$$
u_{\Lambda}\left(\Gamma^{q}\right):=-\ln \frac{\Theta_{\Gamma^{q}}(\Lambda)}{\Theta_{i\left(\Gamma^{q}\right)}(\Lambda)} .
$$

$u_{\Lambda}\left(\Gamma^{q}\right)$ is the free energy cost for introducing the new contour $\Gamma^{q}$ in the restricted model where all contours satisfy $\Gamma^{\prime q} \leq i\left(\Gamma^{q}\right)$.

II. Let

$$
\left[u_{\Lambda}\left(\Gamma^{q}\right)\right]_{\mu^{*}}^{(k)}:=\left.\frac{\mathrm{d}^{k}}{\mathrm{~d} \mu^{k}} u_{\Lambda}\left(\Gamma^{q}\right)(\mu)\right|_{\mu=\mu^{*}} .
$$

The derivative $\left[u_{\Lambda}\left(\Gamma^{q}\right)\right]_{\mu^{*}}^{(k)}$ is estimated using Cauchy formula. In step II one proves good analyticity domains for the functions $\left[u_{\Lambda}\left(\Gamma^{q}\right)\right]_{\mu^{*}}^{(k)}$. It is not very difficult to show that the weight $\omega\left(\Gamma^{q}\right)$ has an analytic continuation in a disc of radius $O\left(V\left(\Gamma^{q}\right)^{-\frac{1}{d}}\right)$ centered at $\mu^{*}$. This is not sufficient. The size of the neighbourhoods of $\mu^{*}$, where the contours are stable and analytic functions of $z$, is related directly to the best constant of variational problems of isoperimetric type. More specifically, for each $n \in \mathbb{N}$, there is a variational problem ${ }^{32}$ for each label $q$ : find the isoperimetric constant $\chi_{q}(n)$ defined by

$$
\chi_{q}(n)^{-1}:=\inf \left\{C: \frac{V\left(\Gamma^{q}\right)^{\frac{d-1}{d}}}{\left\|\Gamma^{q}\right\|} \leq C, \forall \Gamma^{q} \text { such that } V\left(\Gamma^{q}\right) \geq n\right\} .
$$

Hence

$$
V\left(\Gamma^{q}\right)^{\frac{d-1}{d}} \leq \chi_{q}\left(V\left(\Gamma^{q}\right)\right)^{-1}\left\|\Gamma^{q}\right\|
$$

\footnotetext{
${ }^{32}$ These variational problems are not those considered by Isakov.
} 
The constants $\chi_{q}(n)$ form a bounded increasing sequence, and

$$
\chi_{q}(\infty):=\lim _{n} \chi_{q}(n)
$$

One knows very little about the variational problems (4.4). The strategy is to avoid discussing them, as much as possible. One introduces

$$
R_{q}(n):=\inf _{m: m \leq n} \frac{\chi_{q}(m)}{m^{\frac{1}{d}}},
$$

whose main property is that $n \mapsto n R_{q}(n)$ is increasing in $n$.

Lemma 4.1. For any $\chi_{q}^{\prime}<\chi_{q}(\infty)$, there exists $N\left(\chi_{q}^{\prime}\right)$ such that for all $n \geq N\left(\chi_{q}^{\prime}\right)$,

$$
\frac{\chi_{q}^{\prime}}{n^{\frac{1}{d}}} \leq R_{q}(n) \leq \frac{\chi_{q}(\infty)}{n^{\frac{1}{d}}} .
$$

For $q=1,2, n \mapsto n R_{q}(n)$ is increasing in $n$.

Proof. See subsection 4.2 .

The next proposition gives the domains of analyticity and the stability properties of the weights $\omega\left(\Gamma^{q}\right)$ needed for estimating the derivatives of the pressure. Its proof is based on the method of proof of Proposition 3.1. Recall that $\tau_{\star}(\beta)=\beta\left(\rho\left(1-\theta^{\star}\right)-\varepsilon\right)$.

Proposition 4.1. Let $0<\theta<1, \theta<\theta^{\star}<1$, and $0<\varepsilon<1$, so that

$$
\rho\left(1-\theta^{\star}\right)-\varepsilon>0 .
$$

Then there exists $\beta_{0}^{\prime} \geq \beta_{0}$, such that for all $\beta \geq \beta_{0}^{\prime} \omega\left(\Gamma^{2}\right)$ is analytic and $\tau_{\star}(\beta)$-stable in a complex neighborhood of

$$
\left\{z \in \mathbb{C}: \operatorname{Re} z \leq \mu^{*}(\operatorname{Im} z ; \beta)+\theta \Delta^{-1} R_{2}\left(V\left(\Gamma^{2}\right)\right)\right\} \cap \mathbb{U}_{0} .
$$

Moreover

$$
\left|\frac{d}{d z} \omega\left(\Gamma^{2}\right)\right| \leq \mathrm{e}^{-\tau_{\star}(\beta)\left|\Gamma^{2}\right|} .
$$

Similar properties hold for $\omega\left(\Gamma^{1}\right)$ in a complex neighborhood of

$$
\left\{z \in \mathbb{C}: \mu^{*}(\operatorname{Im} z ; \beta)-\theta \Delta^{-1} R_{1}\left(V\left(\Gamma^{1}\right)\right) \leq \operatorname{Re} z\right\} \cap \mathbb{U}_{0} .
$$

Proof. See subsection 4.2 .

III. In the third step one re-writes the derivatives of the pressure $g_{\Lambda}^{q}$ in a convenient way. The $\mathrm{k}^{\text {th }}$-derivative of $g_{\Lambda}^{q}$ at $\mu^{*}$ is

$$
\left[g_{\Lambda}^{q}\right]_{\mu^{*}}^{(k)}=-\frac{1}{\beta|\Lambda|} \sum_{\Gamma^{q} \subset \Lambda}\left[u_{\Lambda}\left(\Gamma^{q}\right)\right]_{\mu^{*}}^{(k)} .
$$


One first writes $u_{\Lambda}\left(\Gamma^{q}\right)$ as follows.

$$
\begin{aligned}
\Theta_{\Gamma^{q}}(\Lambda) & =\Theta_{i\left(\Gamma^{q}\right)}(\Lambda)+\omega\left(\Gamma^{q}\right) \Theta_{i\left(\Gamma^{q}\right)}\left(\Lambda\left(\Gamma^{q}\right)\right) \\
& =\Theta_{i\left(\Gamma^{q}\right)}(\Lambda)\left(1+\omega\left(\Gamma^{q}\right) \frac{\Theta_{i\left(\Gamma^{q}\right)}\left(\Lambda\left(\Gamma^{q}\right)\right)}{\Theta_{i\left(\Gamma^{q}\right)}(\Lambda)}\right) .
\end{aligned}
$$

In this expression $\Theta_{i\left(\Gamma^{q}\right)}\left(\Lambda\left(\Gamma^{q}\right)\right)$ denotes the partition function

$$
\Theta_{i\left(\Gamma^{q}\right)}\left(\Lambda\left(\Gamma^{q}\right)\right):=1+\sum \prod_{i=1}^{n} \omega\left(\Gamma_{i}^{q}\right),
$$

where the sum is over all families of compatible contours $\left\{\Gamma_{1}^{q}, \ldots, \Gamma_{n}^{q}\right\}$, $\Gamma_{i}^{q} \leq i\left(\Gamma^{q}\right), i=1, \ldots, n$, and such that $\left\{\Gamma^{q}, \Gamma_{1}^{q}, \ldots, \Gamma_{n}^{q}\right\}$ is a compatible family. Set

$$
\phi_{\Lambda}\left(\Gamma^{q}\right):=\omega\left(\Gamma^{q}\right) \frac{\Theta_{i\left(\Gamma^{q}\right)}\left(\Lambda\left(\Gamma^{q}\right)\right)}{\Theta_{i\left(\Gamma^{q}\right)}(\Lambda)} .
$$

With these notations

$$
u_{\Lambda}\left(\Gamma^{q}\right)=-\ln \left(1+\phi_{\Lambda}\left(\Gamma^{q}\right)\right)=\sum_{n \geq 1} \frac{(-1)^{n}}{n} \phi_{\Lambda}\left(\Gamma^{q}\right)^{n} .
$$

Let $q=2 .\left[\phi_{\Lambda}\left(\Gamma^{2}\right)^{n}\right]_{\mu^{*}}^{(k)}$ is computed using Cauchy formula,

$$
\left[\phi_{\Lambda}\left(\Gamma^{2}\right)^{n}\right]_{\mu^{*}}^{(k)}=\frac{k !}{2 \pi i} \oint_{\partial D_{r}} \frac{\phi_{\Lambda}\left(\Gamma^{2}\right)^{n}(z)}{\left(z-\mu^{*}\right)^{k+1}} d z,
$$

where $\partial D_{r}$ is the boundary of a disc $D_{r}$ of radius $r$ and center $\mu^{*}$ inside the analyticity region of Proposition 4.1,

$$
\mathbb{U}_{0} \cap\left\{z \in \mathbb{C}: \operatorname{Re} z \leq \mu^{*}(\operatorname{Im}(z) ; \beta)+\theta \Delta^{-1} R_{2}\left(V\left(\Gamma^{2}\right)\right)\right\} .
$$

The function $z \mapsto \frac{\phi_{\Lambda}\left(\Gamma^{2}\right)^{n}(z)}{\left(z-\mu^{*}\right)^{k+1}}$ is real on the real axis, so that

$$
\overline{\left(\frac{\phi_{\Lambda}\left(\Gamma^{2}\right)^{n}(\bar{z})}{\left(\bar{z}-\mu^{*}\right)^{k+1}}\right)}=\frac{\phi_{\Lambda}\left(\Gamma^{2}\right)^{n}(z)}{\left(z-\mu^{*}\right)^{k+1}} .
$$

Consequently

$$
\frac{k !}{2 \pi i} \oint_{\partial D_{r}} \frac{\phi_{\Lambda}\left(\Gamma^{2}\right)^{n}(z)}{\left(z-\mu^{*}\right)^{k+1}} d z=\operatorname{Re}\left\{\frac{k !}{2 \pi i} \oint_{\partial D_{r}} \frac{\phi_{\Lambda}\left(\Gamma^{2}\right)^{n}(z)}{\left(z-\mu^{*}\right)^{k+1}} d z\right\} .
$$

Assuming $^{33}$ that the disc $D_{r}$ is inside the analyticity region of $\omega\left(\Gamma^{2}\right)$, one decomposes $\partial D_{r}$ into

$$
\partial D_{r}^{g}:=\partial D_{r} \cap\left\{z: \operatorname{Re} z \leq \mu^{*}(\operatorname{Im}(z) ; \beta)-\theta \Delta^{-1} R_{1}\left(V\left(\Gamma^{2}\right)\right)\right\}
$$

\footnotetext{
${ }^{33}$ From (3.27) it follows that there exists $C^{\prime}$ independent of $\nu$ and $n$, such that $\mu_{n}^{*}(\nu ; \beta) \geq \mu_{n}^{*}(0 ; \beta)-C^{\prime} \nu^{2}$.
}

This implies that the disc $D_{r}$ of center $\mu^{*}$ and radius $r=\theta \Delta^{-1} R_{2}\left(V\left(\Gamma^{2}\right)\right)$ is inside the analyticity region of $\omega\left(\Gamma^{2}\right)$, provided that $V\left(\Gamma^{2}\right)$ is large enough. 
and

$$
\partial D_{r}^{d}:=\partial D_{r} \cap\left\{z: \operatorname{Re} z \geq \mu^{*}(\operatorname{Im}(z) ; \beta)-\theta \Delta^{-1} R_{1}\left(V\left(\Gamma^{2}\right)\right)\right\} .
$$

One writes (4.8) as a sum of two integrals $I_{k, n}^{g}\left(\Gamma^{2}\right)$ and $I_{k, n}^{d}\left(\Gamma^{2}\right)$,

$$
I_{k, n}^{g}\left(\Gamma^{2}\right):=\operatorname{Re}\left\{\frac{k !}{2 \pi i} \oint_{\partial D_{r}^{g}} \frac{\phi_{\Lambda}\left(\Gamma^{2}\right)^{n}(z)}{\left(z-\mu^{*}\right)^{k+1}} d z\right\}
$$

and

$$
I_{k, n}^{d}\left(\Gamma^{2}\right):=\operatorname{Re}\left\{\frac{k !}{2 \pi i} \oint_{\partial D_{r}^{d}} \frac{\phi_{\Lambda}\left(\Gamma^{2}\right)^{n}(z)}{\left(z-\mu^{*}\right)^{k+1}} d z\right\} .
$$

An analogous decomposition holds for $\Gamma^{1}$ instead of $\Gamma^{2}$.

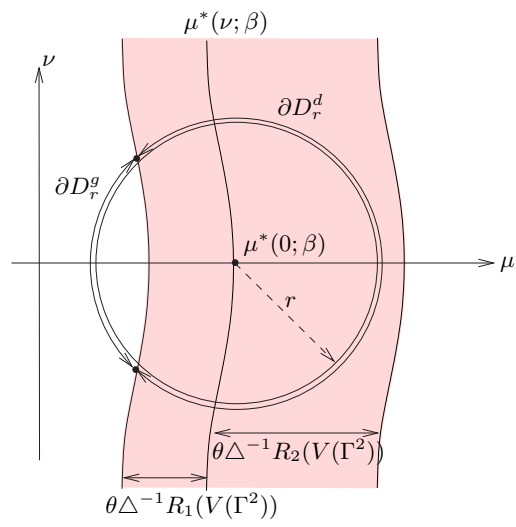

Figure 3: The decomposition of the integral into $I_{k, n}^{g}\left(\Gamma^{2}\right)$ and $I_{k, n}^{d}\left(\Gamma^{2}\right)$

IV. The core of the proof is that $\left[\phi_{\Lambda}\left(\Gamma^{q}\right)^{n}\right]_{\mu^{*}}^{(k)}$ can be estimated very accurately, for large $k$, provided that $V\left(\Gamma^{q}\right)$ is large: there exists $k_{0} \in \mathbb{N}$, and for each contour $\Gamma^{q}$ an integer $k_{+}^{q}\left(\Gamma^{q}\right) \geq k_{0}$ (see (4.17)), such that for each $k \in\left[k_{0}, k_{+}^{q}\left(\Gamma^{q}\right)\right],\left[\phi_{\Lambda}\left(\Gamma^{q}\right)^{n}\right]_{\mu^{*}}^{(k)}$ can be estimated uniformly in $\Lambda$ by the stationary phase method. The function $k_{+}^{q}\left(\Gamma^{q}\right)$ has the following properties

$$
k_{+}^{q}\left(\Gamma_{1}^{q}\right)<k_{+}^{q}\left(\Gamma_{2}^{q}\right) \text {, if } V\left(\Gamma_{1}^{q}\right)<V\left(\Gamma_{2}^{q}\right)
$$

and

$$
\lim _{V\left(\Gamma^{q}\right) \rightarrow \infty} k_{+}^{q}\left(\Gamma^{q}\right)=\infty
$$


Fix $k>k_{0}$. Using the function $k_{+}^{q}\left(\Gamma^{q}\right)$ one defines ${ }^{34}$

$$
k \text {-large contours, if } k \leq k_{+}^{q}\left(\Gamma^{q}\right)
$$

and

$$
k \text {-small contours, if } k>k_{+}^{q}\left(\Gamma^{q}\right) .
$$

The contribution of $\left[u_{\Lambda}\left(\Gamma^{q}\right)\right]_{\mu^{*}}^{(k)}$ to the $\mathrm{k}^{\text {th }}$-derivative of the pressure, when $\Gamma^{q}$ is a large contour, is controlled uniformly in the box $\Lambda$, because for those contours one controls $\left[\phi_{\Lambda}\left(\Gamma^{q}\right)^{n}\right]_{\mu^{*}}^{(k)}$ uniformly in $\Lambda$. On the other hand, for small contours one gets only upper bounds. Let

$$
s_{\Lambda}^{q, k}=\frac{1}{\beta|\Lambda|} \sum_{\substack{\Gamma^{q} \subset \Lambda \\ \Gamma^{q} k \text {-small }}} u_{\Lambda}\left(\Gamma^{q}\right) .
$$

By Cauchy formula

$$
\left|\left[s_{\Lambda}^{q, k}\right]_{\mu^{*}}^{(k)}\right|=\left|\frac{k !}{2 \pi i} \oint_{\partial D_{r}} \frac{s_{\Lambda}^{q, k}(z)}{\left(z-\mu^{*}\right)^{k+1}} d z\right| \leq \frac{k !}{r^{k}} \sup _{z \in \partial D_{r}}\left|s_{\Lambda}^{q, k}(z)\right|,
$$

where the disc $D_{r}$ of center $\mu^{*}$ and radius $r$ is taken as large as possible, according to Proposition 4.1. For details see point $\mathbf{V}$ below.

I consider now the specific case $q=2$. To estimate $\left[\phi_{\Lambda}\left(\Gamma^{2}\right)^{n}\right]_{\mu^{*}}^{(k)}$ one estimates separately $I_{k, n}^{g}\left(\Gamma^{2}\right)$ and $I_{k, n}^{d}\left(\Gamma^{2}\right) . I_{k, n}^{g}\left(\Gamma^{2}\right)$ is not the main contribution to (4.8), so that it is sufficient to get an upper bound for this integral. Let $z \in \mathbb{U}_{0}$ and $\operatorname{Re} z \leq \mu^{*}(\operatorname{Im}(z) ; \beta)$. From (3.25) one gets

$$
\left|\omega\left(\Gamma^{2}\right)\right| \leq \exp \left[-\beta\left\|\Gamma^{2}\right\|+\beta|\operatorname{Re} z| C_{1}\left|\Gamma^{2}\right|+3 C_{0} \delta\left|\Gamma^{2}\right|\right] .
$$

Using formula (3.10), one gets after cancellation and the use of Lemma 3.2 and Proposition 4.1 (see also (3.9)),

$$
\left|\frac{\Theta_{i\left(\Gamma^{2}\right)}\left(\Lambda\left(\Gamma^{2}\right)\right)}{\Theta_{i\left(\Gamma^{2}\right)}(\Lambda)}\right| \leq \mathrm{e}^{\delta\left|\overline{\Gamma^{2}}\right|} \leq \mathrm{e}^{\delta C_{5}\left|\Gamma^{2}\right|} .
$$

Set

$$
\zeta:=z-\mu^{*} .
$$

There exists a constant $C_{6}$ so that

$$
\left|\phi_{\Lambda}\left(\Gamma^{2}\right)\right| \leq \mathrm{e}^{-\beta\left\|\Gamma^{2}\right\|\left(1-C_{6} \delta-|\operatorname{Re} \zeta| C_{1} \rho^{-1}\right)} \quad \text { if } \quad \operatorname{Re} \zeta \leq \mu^{*}(\operatorname{Im}(\zeta) ; \beta)-\mu^{*} .
$$

This upper bound implies that

$$
\left|I_{k, n}^{g}\left(\Gamma^{2}\right)\right| \leq \frac{k !}{r^{k}} \mathrm{e}^{-n \beta\left\|\Gamma^{2}\right\|\left(1-C_{6} \delta-r C_{1} \rho^{-1}\right)} .
$$

\footnotetext{
${ }^{34}$ Later (see point $\mathbf{V}$ below) one must make a finer distinction, and one distinguishes large and thin contours and large and fat contours. The important contours are the large and thin contours.
} 
To estimate $I_{k, n}^{d}\left(\Gamma^{2}\right)$ one first proves Lemma 4.2 and then performs a stationary phase analysis (Lemma 4.3). Lemma 4.2 states that $\phi_{\Lambda}\left(\Gamma^{2}\right)$ is almost an exponential function of $\zeta$. From the stationary phase analysis one gets that (see (4.18) and (4.19))

$$
\left[\phi_{\Lambda}\left(\Gamma^{2}\right)\right]_{\mu^{*}}^{(k)} \sim\left(\beta \Delta V\left(\Gamma^{2}\right)\right)^{k} \phi_{\Lambda}\left(\Gamma^{2}\right)\left(\mu^{*}\right) .
$$

Lemma 4.2. For $\beta$ large enough, there exists an analytic function $\mathrm{g}\left(\Gamma^{2}\right)$ of $\zeta$ in a neighborhood of $\zeta=0$,

$$
\begin{aligned}
\mu^{*}(\operatorname{Im}(\zeta) ; \beta)+\mu^{*}-\theta \Delta^{-1} R_{1}\left(V\left(\Gamma^{2}\right)\right) \leq \operatorname{Re} \zeta \\
\leq \mu^{*}(\operatorname{Im}(\zeta) ; \beta)+\mu^{*}+\theta \Delta^{-1} R_{2}\left(V\left(\Gamma^{2}\right)\right),
\end{aligned}
$$

such that $\mathrm{g}\left(\Gamma^{2}\right)(0)=0$ and

$$
\phi_{\Lambda}\left(\Gamma^{2}\right)\left(\mu^{*}+\zeta\right)=\phi_{\Lambda}\left(\Gamma^{2}\right)\left(\mu^{*}\right) \mathrm{e}^{\beta \Delta V\left(\Gamma^{2}\right)\left(\zeta+\mathrm{g}\left(\Gamma^{2}\right)(\zeta)\right)} .
$$

For suitable constants $C_{7}$ and $C_{8}$,

$$
\left|\frac{d}{d \zeta} \mathrm{g}\left(\Gamma^{2}\right)(\zeta)\right| \leq C_{7} \mathrm{e}^{-\tau_{\star}(\beta)}+C_{8} \frac{\left|\Gamma^{2}\right|}{V\left(\Gamma^{2}\right)} .
$$

There exists a constant $C_{9}$ so that

$$
\exp \left[-\beta\left\|\Gamma^{2}\right\|\left(1+C_{9} \delta\right)\right] \leq \phi_{\Lambda}\left(\Gamma^{2}\right)\left(\mu^{*}\right) \leq \exp \left[-\beta\left\|\Gamma^{2}\right\|\left(1-C_{9} \delta\right)\right] .
$$

Proof. See subsection 4.3.

To compute $I_{k, n}^{d}\left(\Gamma^{2}\right)$ one parametrizes $\partial D_{r}^{d}$ by $z:=\mu^{*}+r \mathrm{e}^{i \alpha},-\alpha_{1} \leq$ $\alpha \leq \alpha_{2}$, and $0<\alpha_{i} \leq \pi$. The stationary phase point for the integral $I_{k, n}^{d}\left(\Gamma^{2}\right)$ is determined as follows. Let

$$
c(n):=n \beta \Delta V\left(\Gamma^{2}\right) \quad \text { and } \quad \zeta \equiv r \mathrm{e}^{i \alpha} .
$$

Using (4.12) and the notation $\phi_{\Lambda}^{*}\left(\Gamma^{2}\right) \equiv \phi_{\Lambda}\left(\Gamma^{2}\right)\left(\mu^{*}\right)$,

$$
I_{k, n}^{d}\left(\Gamma^{2}\right)=k ! \frac{\phi_{\Lambda}^{*}\left(\Gamma^{2}\right)^{n}}{2 \pi r^{k}} \int_{-\alpha_{1}}^{\alpha_{2}} \mathrm{e}^{c(n) r \cos \alpha+c(n) \operatorname{Reg}\left(\Gamma^{2}\right)(\zeta)}[\cos (\widetilde{\psi}(\alpha))] d \alpha,
$$

with

$$
\widetilde{\psi}(\alpha):=c(n) r \sin \alpha+c(n) \operatorname{Im} \mathrm{g}\left(\Gamma^{2}\right)(\zeta)-k \alpha .
$$

The stationary phase point denoted by $\zeta_{k, n}=r_{k, n} \mathrm{e}^{i \alpha_{k, n}}$ is solution of the equations

$$
\frac{d}{d \alpha}\left(c(n) r \cos \alpha+c(n) \operatorname{Reg}\left(\Gamma^{2}\right)\left(r \mathrm{e}^{i \alpha}\right)\right)=0 \quad \text { and } \quad \frac{d}{d \alpha} \widetilde{\psi}(\alpha)=0 .
$$


These equations are equivalent to the equations ( $'$ denotes the derivative with respect to $\zeta$ )

$$
\begin{aligned}
\sin \alpha\left(1+\operatorname{Reg}\left(\Gamma^{2}\right)^{\prime}(\zeta)\right)+\cos \alpha \operatorname{Img} g\left(\Gamma^{2}\right)^{\prime}(\zeta) & =0 ; \\
c(n) r\left[\cos \alpha\left(1+\operatorname{Reg}\left(\Gamma^{2}\right)^{\prime}(\zeta)\right)-\sin \alpha \operatorname{Img} g\left(\Gamma^{2}\right)^{\prime}(\zeta)\right] & =k .
\end{aligned}
$$

Since $\mathrm{g}\left(\Gamma^{2}\right)$ is real on the real axis, $\alpha_{k, n}=0$ and $r_{k, n}$ is solution of

$$
c(n) r\left(1+\mathrm{g}\left(\Gamma^{2}\right)^{\prime}(r)\right)=k .
$$

The next lemma gives the result of the standard stationary phase analysis, see e.g. appendix of [I1].

Lemma 4.3. Let $\alpha_{i} \geq \pi / 4, i=1,2, A \leq 1 / 25$ and $c(n) \geq 1$. If $\mathrm{g}(\zeta)$ is analytic in $\zeta$ in the disc $\{\zeta:|\zeta| \leq \rho\}$, real on the real axis, and for all $\zeta$ in that disc

$$
\left|\frac{d}{d \zeta} \mathrm{g}\left(\Gamma^{2}\right)(\zeta)\right| \leq A
$$

then there exists $k_{0}(A) \in \mathbb{N}$, such that for all integers $k$,

$$
k \in\left[k_{0}(A), c(n)(1-2 \sqrt{A}) \rho\right],
$$

there is a unique solution $0<r_{k, n}<\rho$ of (4.16). Moreover,

$$
\begin{aligned}
\frac{\mathrm{e}^{c(n) r_{k, n}+c(n) \mathrm{g}\left(\Gamma^{2}\right)\left(r_{k, n}\right)}}{10 \sqrt{c(n) r_{k, n}}} & \leq \frac{1}{2 \pi} \int_{-\alpha_{1}}^{\alpha_{2}} \mathrm{e}^{c(n) r \cos \alpha+c(n) \operatorname{Reg}\left(\Gamma^{2}\right)}[\cos (\widetilde{\psi}(\alpha))] d \alpha \\
& \leq \frac{\mathrm{e}^{c(n) r_{k, n}+c(n) \mathrm{g}\left(\Gamma^{2}\right)\left(r_{k, n}\right)}}{\sqrt{c(n) r_{k, n}}} .
\end{aligned}
$$

Proof. Existence and uniqueness of $r_{k, n}$ is a consequence of the monotonicity of $r \mapsto c(n) r\left(1+\mathrm{g}\left(\Gamma^{2}\right)^{\prime}(r)\right)$.

According to Lemmas 4.3 and 4.2 , let

$$
k_{+}\left(\Gamma^{2} \mid \theta, A, \beta\right):=\theta(1-2 \sqrt{A}) \beta V\left(\Gamma^{2}\right) R_{2}\left(V\left(\Gamma^{2}\right)\right) .
$$

Lemma 4.3 implies that for all

$$
k \in\left[k_{0}(A), k_{+}\left(\Gamma^{2} \mid \theta, A, \beta\right)\right],
$$

one can find a solution $r_{k, n}$ for any $n \geq 1$, so that (see (4.16))

$$
k-\frac{k A}{(1+A)}=\frac{k}{(1+A)} \leq c(n) r_{k, n} \leq \frac{k}{(1-A)}=k+\frac{k A}{(1-A)},
$$

and

$$
c(n)\left|\mathrm{g}\left(\Gamma^{2}\right)\left(r_{k, n}\right)\right|=c(n)\left|\int_{0}^{r_{k, n}} \mathrm{~g}\left(\Gamma^{2}\right)^{\prime}(\zeta) d \zeta\right| \leq A c(n) r_{k, n} \leq k \frac{A}{1-A} .
$$


Therefore, setting

$$
c_{+}(A)=(1+A) \exp \left[\frac{2 A}{1-A}\right] \quad \text { and } \quad c_{-}(A)=(1-A) \exp \left[-\frac{2 A}{1-A^{2}}\right]
$$

one gets (see (4.15))

$$
\begin{aligned}
I_{k, n}^{d}\left(\Gamma^{2}\right) & \leq \sqrt{2 \pi(1+A)} \mathrm{e}^{\frac{1}{12 k}} c_{+}^{k} c(n)^{k} \phi_{\Lambda}^{*}\left(\Gamma^{2}\right)^{n} \\
& \stackrel{A \rightarrow 0}{\longrightarrow} \sqrt{2 \pi} \mathrm{e}^{\frac{1}{12 k}} c(n)^{k} \phi_{\Lambda}^{*}\left(\Gamma^{2}\right)^{n}
\end{aligned}
$$

and

$$
\begin{aligned}
I_{k, n}^{d}\left(\Gamma^{2}\right) & \geq \frac{\sqrt{2 \pi(1-A)}}{10} c_{-}^{k} c(n)^{k} \phi_{\Lambda}^{*}\left(\Gamma^{2}\right)^{n} \\
& \stackrel{A \rightarrow 0}{\longrightarrow} \frac{\sqrt{2 \pi}}{10} c(n)^{k} \phi_{\Lambda}^{*}\left(\Gamma^{2}\right)^{n} .
\end{aligned}
$$

V. The next step is to estimate $\left[u_{\Lambda}\left(\Gamma^{2}\right)\right]_{\mu^{*}}^{(k)}$ from the previous estimates. The main result is that for a subclass of $k$-large contours ( $k$-large and thin contours), if $k$ is sufficiently large, $-\left[u_{\Lambda}\left(\Gamma^{2}\right)\right]_{\mu^{*}}^{(k)}$ has always the same sign and there exists a positive function $D(k)$ such that $\lim _{k \rightarrow \infty} D(k)=0$ and

$$
-\left[u_{\Lambda}\left(\Gamma^{2}\right)\right]_{\mu^{*}}^{(k)} \geq \frac{1}{20}(1-D(k))\left(\beta \Delta V\left(\Gamma^{2}\right)\right)^{k} c_{-}^{k} \phi_{\Lambda}^{*}\left(\Gamma^{2}\right) .
$$

This analysis is not difficult, but it must be done carefully. The principal points are here emphasized, complete details are given in subsection 4.2 of [FrPf1].

The parameters $\theta$ and $A$ are not yet fixed. Two new parameters are introduced, $\varepsilon^{\prime}$ and later $\eta$ in Definition 4.1. It is important to see that one can choose these parameters in a consistent manner. Let $0<\theta<1$, $A \leq 1 / 25$, and set

$$
\hat{\theta}:=\theta(1-2 \sqrt{A})
$$

Let $\varepsilon^{\prime}>0$ and $\chi_{2}^{\prime}$ so that

$$
\left(1+\varepsilon^{\prime}\right) \chi_{2}^{\prime}>\chi_{2}(\infty)
$$

I fix the values of $\theta$, and $\varepsilon^{\prime}$ by the following conditions. I choose $0<A_{0}<$ $1 / 25$; the parameters $\theta$ and $\varepsilon^{\prime}$ are chosen so that

$$
\frac{\mathrm{e}^{\frac{1}{d}}}{\theta\left(1-2 \sqrt{A_{0}}\right)}<\frac{d}{d-1} \frac{c_{-}\left(A_{0}\right)^{\frac{d-1}{d}}}{1+\varepsilon^{\prime}} \text { and } \frac{1-2 \sqrt{A_{0}}}{1+\varepsilon^{\prime}} \frac{d}{d-1}>1 .
$$

This is possible, because ${ }^{35}$

$$
\frac{d}{(d-1)}>\mathrm{e}^{\frac{1}{d}}
$$

\footnotetext{
${ }^{35} d \mathrm{e}^{\frac{1}{d}-1}=d \mathrm{e}^{\frac{1}{d}-1-\frac{1}{d}+\frac{1}{d}}=\sum_{n \geq 2} \frac{1}{n !} \frac{1}{d}^{n-1}+1<1-\frac{1}{2 d}+\sum_{n \geq 1} \frac{1}{n !} \frac{1}{d}^{n}=$ $\mathrm{e}^{\frac{1}{d}}-\frac{1}{2 d}$.
} 
Given $\theta$, the value of $\theta^{\star}$ is fixed in Proposition 4.1. From now on the values of $\theta, \theta^{\star}$ and $\varepsilon^{\prime}$ are fixed once for all.

Notice that conditions (4.21) are still satisfied with the same values of $\theta$ and $\varepsilon^{\prime}$ if one replaces in (4.21) $A_{0}$ by $0<A<A_{0}$. This means that one is still free to choose $A<A_{0}$.

Given $k$, there is a natural distinction between contours $\Gamma^{2}$, according to the fact that $k \geq k_{+}\left(\Gamma^{2} \mid \theta, A, \beta\right)$, or $k<k_{+}\left(\Gamma^{2} \mid \theta, A, \beta\right)$ (see (4.17)).

Definition 4.1. A contour $\Gamma^{2}$ is a

1. $k$-small contour, if $\hat{\theta} \beta V\left(\Gamma^{2}\right) R_{2}\left(V\left(\Gamma^{2}\right)\right) \leq k$;

2. fat contour, if for $\eta \geq 0, V\left(\Gamma^{2}\right)^{\frac{d-1}{d}} \leq \eta\left\|\Gamma^{2}\right\|$;

3. $k$-large and thin contour, if $\hat{\theta} \beta V\left(\Gamma^{2}\right) R_{2}\left(V\left(\Gamma^{2}\right)\right)>k, V\left(\Gamma^{2}\right)^{\frac{d-1}{d}}>$ $\eta\left\|\Gamma^{2}\right\|$.

The parameter $\eta$ is fixed below.

It is sufficient to consider large values of $k$. This is implicitly assumed below. It is not difficult to estimate the derivative of $u_{\Lambda}\left(\Gamma^{2}\right)$ by Cauchy formula with a disc of center $\mu^{*}$ and radius $\Delta^{-1}\left(\hat{\theta} \chi_{2}^{\prime}\right)^{\frac{d}{d-1}} \beta^{\frac{1}{d-1}} k^{-\frac{1}{d-1}}$. There exists a constant $C_{10}$ such that

$$
\left|\sum_{\substack{\Gamma^{2}: \text { Int } \Gamma^{2} \ni 0 \\ \Gamma^{2} k \text {-small }}}\left[u_{\Lambda}\left(\Gamma^{2}\right)\right]_{\mu^{*}}^{(k)}\right| \leq C_{10}\left(\frac{\Delta}{\beta^{\frac{1}{d-1}}\left(\hat{\theta} \chi_{2}^{\prime}\right)^{\frac{d}{d-1}}}\right)^{k} k ! k^{\frac{k}{d-1}} .
$$

Moreover, if one chooses $\eta$ small enough, then the contribution of fat contours (which are not $k$-small) is negligible compare to the contribution of the small contours. The value of $\eta$ is chosen so that this is the case.

The $k$-large and thin contours are the important contours. For them one has lower and upper bounds for $\left[\phi_{\Lambda}\left(\Gamma^{2}\right)^{n}\right]_{\mu^{*}}^{(k)}$. Using these bounds one gets lower (and upper) bounds on $-\left[u_{\Lambda}\left(\Gamma^{2}\right)\right]_{\mu^{*}}^{(k)}$. There are two cases.

I. Assume that $R_{1}\left(V\left(\Gamma^{2}\right)\right) \geq R_{2}\left(V\left(\Gamma^{2}\right)\right)$, or that $V\left(\Gamma^{2}\right)$ is so large that

$$
\hat{\theta} \beta V\left(\Gamma^{2}\right) R_{1}\left(V\left(\Gamma^{2}\right)\right)>k .
$$

Under these conditions one can apply Lemma 4.3 with a disc $D_{r_{k, n}}$ so that $\partial D_{r_{k, n}}=\partial D_{r_{k, n}}^{d}$. Indeed, either $R_{1}\left(V\left(\Gamma^{2}\right)\right) \geq R_{2}\left(V\left(\Gamma^{2}\right)\right)$, and then one applies Lemma 4.3 with $R=\theta \Delta^{-1} R_{2}\left(V\left(\Gamma^{2}\right)\right)$, or this is not true, but the other condition is valid, so that one chooses $R=\theta \Delta^{-1} R_{1}\left(V\left(\Gamma^{2}\right)\right)$. In both cases $r_{k, n}<R$, which implies $\partial D_{r_{k, n}}=\partial D_{r_{k, n}}^{d}$. From the estimates for $\left[\phi_{\Lambda}\left(\Gamma^{2}\right)^{n}\right]_{\mu^{*}}^{(k)}$, one obtains 
Lemma 4.4. There exists a function $D(k), \lim _{k \rightarrow \infty} D(k)=0$, such that for $\beta$ sufficiently large and $A$ sufficiently small the following holds. If $k$ is large enough and $R_{1}\left(V\left(\Gamma^{2}\right)\right) \geq R_{2}\left(V\left(\Gamma^{2}\right)\right)$ or $\hat{\theta} \beta V\left(\Gamma^{2}\right) R_{1}\left(V\left(\Gamma^{2}\right)\right)>k$, then

$$
-\left[u_{\Lambda}\left(\Gamma^{2}\right)\right]_{\mu^{*}}^{(k)} \geq \frac{1}{5}(1-D(k))\left(\beta \Delta V\left(\Gamma^{2}\right)\right)^{k} c_{-}^{k} \phi_{\Lambda}^{*}\left(\Gamma^{2}\right) .
$$

Proof. See subsection 4.4 .

II. The second case is when

$$
\hat{\theta} \beta V\left(\Gamma^{2}\right) R_{1}\left(V\left(\Gamma^{2}\right)\right) \leq k \leq \hat{\theta} \beta V\left(\Gamma^{2}\right) R_{2}\left(V\left(\Gamma^{2}\right)\right) .
$$

Since the contours are also thin,

$$
\begin{aligned}
\beta\left\|\Gamma^{2}\right\| & \leq \eta^{-1} \hat{\theta}^{-1} \chi_{1}(1)^{-1} \beta \hat{\theta} \chi_{1}(1) V\left(\Gamma^{2}\right)^{\frac{d-1}{d}} \\
& \leq \eta^{-1} \hat{\theta}^{-1} \chi_{1}(1)^{-1} \beta \hat{\theta} V\left(\Gamma^{2}\right) R_{1}\left(V\left(\Gamma^{2}\right)\right) \\
& \leq \eta^{-1} \hat{\theta}^{-1} \chi_{1}(1)^{-1} k \equiv \lambda k .
\end{aligned}
$$

One chooses $R=\beta \Delta^{-1} R_{2}\left(V\left(\Gamma^{2}\right)\right)$ in Lemma 4.3. The integration in (4.8) is decomposed into two parts (see Figure 3 ), and one shows that the contribution from the integration over $\partial D_{r_{k, n}}^{g}$ is negligible for large enough $\beta$ (see (4.11)). At that point one uses the fact that one can choose $A$ small. This fixes the value of $A$. Inequality (4.22) is crucial; it implies that the contribution from the integration over $\partial D_{r_{k, n}}^{d}$ is not too small, because the surface energy of a contour is not too large. Then one gets as for Lemma 4.4

Lemma 4.5. There exists $0<A^{\prime} \leq A_{0}$ such that for all $\beta$ sufficiently large, the following holds. If $k$ is large enough and $\Gamma^{2}$ is a $k$-large and thin contour, then

$$
-\left[u_{\Lambda}\left(\Gamma^{2}\right)\right]_{\mu^{*}}^{(k)} \geq \frac{1}{20}(1-D(k))\left(\beta \Delta V\left(\Gamma^{2}\right)\right)^{k} c_{-}^{k} \phi_{\Lambda}^{*}\left(\Gamma^{2}\right) .
$$

VI. One can now estimate $\left[g_{\Lambda}^{2}\right]_{\mu^{*}}^{\left(k_{n}\right)}$ for a suitable divergent sequence $\left\{k_{n}\right\}$. Two basic facts are used:

(1) $-\left[u_{\Lambda}\left(\Gamma^{2}\right)\right]_{\mu^{*}}^{(k)}>0$ for any $k$-large and thin contour;

(2) by the choice of $\varepsilon^{\prime}$ there exists $n\left(\varepsilon^{\prime}\right)$, such that

$$
\left(1+\varepsilon^{\prime}\right) \chi_{2}^{\prime}>\chi_{2}(\infty) \quad \text { and } \quad \chi_{2}(\infty) \geq \chi_{2}(n) \geq \chi_{2}^{\prime} \text { if } n \geq n\left(\varepsilon^{\prime}\right) .
$$

By definition of the variational problems (4.4), there exists $\Gamma_{n}^{2}, n \geq n\left(\varepsilon^{\prime}\right)$, such that

$$
\lim _{n \rightarrow \infty}\left\|\Gamma_{n}^{2}\right\|=\infty \quad \text { and } \quad V\left(\Gamma_{n}^{2}\right)^{\frac{d-1}{d}} \geq \frac{\left\|\Gamma_{n}^{2}\right\|}{\left(1+\varepsilon^{\prime}\right) \chi_{2}^{\prime}} .
$$


Proposition 4.2. There exists $\beta^{\prime}$ such that for all $\beta>\beta^{\prime}$, the following holds. There exists an increasing diverging sequence $\left\{k_{n}\right\}$; for each $k_{n}$ there exists $\Lambda\left(L_{n}\right)$ such that for all $\Lambda \supset \Lambda\left(L_{n}\right)$

$$
\left[g_{\Lambda}^{2}\right]_{\mu^{*}}^{\left(k_{n}\right)} \geq C_{14}^{k_{n}} k_{n} !^{\frac{d}{d-1}} \Delta^{k_{n}} \beta^{-\frac{k_{n}}{d-1}} \chi_{2}^{\prime-\frac{d k_{n}}{d-1}} .
$$

$C_{14}>0$ is a constant independent of $\beta, k_{n}$ and $\Lambda$.

Proof. One compares the contributions of the small and fat contours with that of the large and thin contours for large enough $k$. The contribution of the small and of the fat contours is at most

$$
C_{10}\left(\Delta \beta^{-\frac{1}{d-1}} \chi_{2}^{\prime-\frac{d}{d-1}}\right)^{k}\left(\frac{\mathrm{e}^{\frac{1}{d}}}{\theta\left(1-\sqrt{A^{\prime}}\right)}\right)^{k \frac{d}{d-1}} k !^{\frac{d}{d-1}} .
$$

The contribution to $\left[g_{\Lambda}^{2}\right]_{\mu^{*}}^{(k)}$ of each large and thin contour is nonnegative. By assumption (4.20) and the definition of the isoperimetric constant $\chi_{2}$, there exists a sequence $\Gamma_{n}^{2}, n \geq 1$, such that

$$
\lim _{n \rightarrow \infty}\left\|\Gamma_{n}^{2}\right\| \rightarrow \infty \quad \text { and } \quad V\left(\Gamma_{n}^{2}\right)^{\frac{d-1}{d}} \geq \frac{\left\|\Gamma_{n}^{2}\right\|}{\left(1+\varepsilon^{\prime}\right) \chi_{2}^{\prime}} .
$$

Since $x^{k \frac{d}{d-1}} \mathrm{e}^{-x}$ has its maximum at $x=k \frac{d}{d-1}$, let

$$
k_{n}:=\left\lfloor\frac{d-1}{d} \beta\left\|\Gamma_{n}^{2}\right\|\right\rfloor
$$

For any $n, \Gamma_{n}^{2}$ is a thin and $k_{n}$-large volume contour, since by (4.21)

$$
\begin{aligned}
\beta\left(1-2 \sqrt{A^{\prime}}\right) V\left(\Gamma^{2}\right) R_{2}\left(V\left(\Gamma^{2}\right)\right) & \geq \beta\left(1-2 \sqrt{A^{\prime}}\right) V\left(\Gamma^{2}\right)^{\frac{d-1}{d}} \chi_{2}^{\prime} \\
& \geq \frac{\left(1-2 \sqrt{A^{\prime}}\right)}{1+\varepsilon^{\prime}} \beta\left\|\Gamma_{n}^{2}\right\| \geq k_{n} .
\end{aligned}
$$

Let $\Lambda \supset \Gamma_{n}^{2}$. Using Lemma 4.5 or Lemma 4.4 one shows that $-\left[u_{\Lambda}\left(\Gamma_{n}^{2}\right)\right]_{\mu^{*}}^{\left(k_{n}\right)}$ is bounded below by

$$
C_{13}\left(\Delta \beta^{-\frac{1}{d-1}} \chi_{2}^{\prime-\frac{d}{d-1}}\right)^{k_{n}}\left(\frac{d}{d-1} \frac{c_{-}\left(A^{\prime}\right)^{\frac{d-1}{d}}}{1+\varepsilon^{\prime}} \mathrm{e}^{-O(\delta)}\right)^{k_{n} \frac{d}{d-1}} k_{n} !^{\frac{d}{d-1}} .
$$

By the choice (4.21), if $\delta$ is small enough, i.e. $\beta$ large enough, then

$$
\frac{\mathrm{e}^{\frac{1}{d}}}{\theta\left(1-2 \sqrt{A^{\prime}}\right)}<\frac{d}{d-1} \frac{c_{-}\left(A^{\prime}\right)^{\frac{d-1}{d}}}{1+\varepsilon^{\prime}} \mathrm{e}^{-O(\delta)} .
$$

Hence the contribution of the small and fat contours is negligible for large $k_{n}$. Let $\Lambda\left(L_{n}\right)$ be a box which contains at least $\left|\Lambda\left(L_{n}\right)\right| / 4$ translates of 
$\Gamma_{n}^{2}$. For any $\Lambda \supset \Lambda\left(L_{n}\right)$, if $k_{n}$ and $\beta$ are large enough, then there exists a constant $C_{14}>0$, independent of $\beta, k_{n}$ and $\Lambda$, such that

$$
\left[g_{\Lambda}^{2}\right]_{\mu^{*}}^{\left(k_{n}\right)} \geq C_{14}^{k_{n}} k_{n} !^{\frac{d}{d-1}} \Delta^{k_{n}} \beta^{-\frac{k_{n}}{d-1}} \chi_{2}^{\prime-\frac{d k_{n}}{d-1}} .
$$

VII. The bound in Proposition 4.2 is uniform in $\Lambda$. Since (see Lemma 2.7 in $[\mathrm{FrPf} 1])$

$$
\lim _{L \rightarrow \infty}\left[g_{\Lambda(L)}^{2}\right]_{\mu^{*}}^{(k)}=\lim _{t \uparrow \mu^{*}}\left[g^{2}\right]_{t}^{(k)},
$$

the same result holds in the thermodynamic limit. This proves Theorem 4.1 .

\subsection{Proof of Lemma 4.1 and Proposition 4.1}

Proof of Lemma 4.1. Let $q=2$ and suppose that

$$
R_{2}(n)=\frac{\chi_{2}(m)}{m^{\frac{1}{d}}} \quad \text { for some } m<n .
$$

Then $R_{2}\left(m^{\prime}\right)=R_{2}(n)$ for all $m \leq m^{\prime} \leq n$. Let $n^{\prime}$ be the largest $n \geq m$ such that

$$
R_{2}(n)=\frac{\chi_{2}(m)}{m^{\frac{1}{d}}} .
$$

One has $n^{\prime}<\infty$, otherwise

$$
0<R_{2}(m)=R_{2}(n) \leq \frac{\chi_{2}(\infty)}{n^{\frac{1}{d}}} \quad \forall n \geq m,
$$

which is impossible. Therefore either

$$
R_{2}\left(n^{\prime}\right)=\frac{\chi_{2}\left(n^{\prime}\right)}{n^{\prime \frac{1}{d}}} \quad \text { or } \quad R_{2}\left(n^{\prime}+1\right)=\frac{\chi_{2}\left(n^{\prime}+1\right)}{\left(n^{\prime}+1\right)^{\frac{1}{d}}},
$$

and for all $k \geq n^{\prime}+1$,

$$
R_{2}(k)=\inf _{m \leq k} \frac{\chi_{2}(m)}{m^{\frac{1}{d}}}=\inf _{n^{\prime} \leq m \leq k} \frac{\chi_{2}(m)}{m^{\frac{1}{d}}} .
$$

Since $\chi_{2}(m)$ is increasing, for all $k \geq n^{\prime}+1$,

$$
R_{2}(k) \geq \inf _{n^{\prime} \leq m \leq k} \frac{\chi_{2}\left(n^{\prime}\right)}{m^{\frac{1}{d}}}=\frac{\chi_{2}\left(n^{\prime}\right)}{k^{\frac{1}{d}}} .
$$

From the above one concludes that there exist infinitely many $n^{\prime}$ so that (4.25) and (4.26) are true. This proves the first statement. 
On an interval of constancy of $R_{2}(n), n \mapsto n^{a} R_{2}(n)$ is increasing. On the other hand, if

$$
R_{2}(n)=\frac{\chi_{2}(n)}{n^{\frac{1}{d}}} \quad \text { for } n \in\left[m_{1}, m_{2}\right]
$$

then $n \mapsto n R_{2}(n)$ is increasing on $\left[m_{1}, m_{2}\right]$ since $n \mapsto \chi_{2}(n)$ and $n \mapsto n^{1-\frac{1}{d}}$ are increasing.

Proof of Proposition 4.1. $\omega\left(\Gamma^{2}\right)$ is $\tau(\beta)$-stable if $\operatorname{Re} z \leq \mu^{*}(\nu ; \beta) \cap \mathbb{U}_{0}$, and $\frac{d}{d z} \omega\left(\Gamma^{2}\right)$ is $\tau^{\prime}(\beta)$-stable on the same region (Propositions 3.1 and 3.2 ). Similar statements hold for $\omega\left(\Gamma^{1}\right)$ on $\operatorname{Re} z \geq \mu^{*}(\nu ; \beta) \cap \mathbb{U}_{0}$. Let

$$
I_{n}(\nu ; \beta):=\left(\mu^{*}(\nu ; \beta)-\theta \Delta^{-1} R_{1}(n), \mu^{*}(\nu ; \beta)+\theta \Delta^{-1} R_{2}(n)\right) .
$$

As in the proof of $\mathrm{B}$ and $\mathrm{C}$ of Proposition 3.1, one proves by iteration, that on the intervals $I_{n}(\nu ; \beta), \omega\left(\Gamma^{q}\right)$ and $\frac{d}{d z} \omega\left(\Gamma^{q}\right)$ are $\tau_{\star}(\beta)$-stable.

To prove the stability of $\omega\left(\Gamma^{q}\right)$ one verifies (3.16) and (3.17) for some $\theta^{\prime}<\theta^{\star}$. Suppose that the statement is correct for $V\left(\Gamma^{q}\right) \leq n-1$. $\delta=\delta(\beta)$ is defined by (3.13). Let $V\left(\Gamma^{2}\right)=n, z=\mu+i \nu$, and $\mu \geq \mu^{*}(\nu ; \beta)$. Then

$$
\begin{aligned}
\operatorname{Re}\left(p_{1}^{n-1}(z)-p_{2}^{n-1}(z)\right) & \frac{V\left(\Gamma^{2}\right)}{\left\|\Gamma^{2}\right\|}=\operatorname{Re} \int_{\mu_{n}^{*}}^{\mu} \frac{d}{d \mu}\left(p_{1}^{n-1}(z)-p_{2}^{n-1}(z)\right) \frac{V\left(\Gamma^{2}\right)}{\left\|\Gamma^{2}\right\|} \\
& \leq(\Delta+2 \delta)\left(\left|\mu-\mu^{*}\right|+\left|\mu^{*}-\mu_{n}^{*}\right|\right) \frac{V\left(\Gamma^{2}\right)^{\frac{d-1}{d}}}{\left\|\Gamma^{2}\right\|} V\left(\Gamma^{2}\right)^{\frac{1}{d}} \\
& \leq(\Delta+2 \delta)\left(\left|\mu-\mu^{*}\right|+\left|\mu^{*}-\mu_{n}^{*}\right|\right) \frac{n^{\frac{1}{d}}}{\chi_{2}(n)} \\
& \leq(\Delta+2 \delta)\left(\left|\mu-\mu^{*}\right| \frac{1}{R_{2}(n)}+\left|\mu^{*}-\mu_{n}^{*}\right| \frac{n^{\frac{1}{d}}}{\chi_{2}(n)}\right) \\
& \leq \frac{\Delta+2 \delta}{\Delta} \theta+\frac{2(\Delta+2 \delta)}{\beta(\Delta-2 \delta)} \frac{\delta^{l(n)} n^{\frac{1}{d}}}{\chi_{2}(n)} .
\end{aligned}
$$

(3.28) is used for controlling $\left|\mu^{*}-\mu_{n}^{*}\right|$. If $\beta$ is large enough, there exists $\theta<\theta^{\prime}<\theta^{\star}$, independent of $n$, so that

$$
\operatorname{Re}\left(p_{1}^{n-1}(z)-p_{2}^{n-1}(z)\right) \frac{V\left(\Gamma^{2}\right)}{\left\|\Gamma^{2}\right\|} \leq \theta^{\prime} .
$$

The stability of $\frac{d}{d z} \omega\left(\Gamma^{2}\right)$ is a consequence of (use (3.15) for controlling $\left.\left|\omega_{n}\left(\Gamma^{q}\right)\right|\right)$

$$
\begin{aligned}
\left|\frac{d}{d z} \omega\left(\Gamma^{2}\right)\right| & \leq \beta\left|\omega\left(\Gamma^{2}\right)\right|\left(\left|\Gamma^{2}\right|\left(C_{1}+2 \delta C_{0}\right)+V\left(\Gamma^{2}\right)(\Delta+2 \delta)\right) \\
& \leq \beta C_{3}\left|\Gamma^{q}\right|^{\frac{d}{d-1}}\left|\omega\left(\Gamma^{2}\right)\right| .
\end{aligned}
$$




\subsection{Proof of Lemma 4.2}

Proof of Lemma 4.2. Let

$\mu^{*}(\operatorname{Im}(z) ; \beta)-\theta \Delta^{-1} R_{1}\left(V\left(\Gamma^{2}\right)\right) \leq \operatorname{Re} z \leq \mu^{*}(\operatorname{Im}(z) ; \beta)+\theta \Delta^{-1} R_{2}\left(V\left(\Gamma^{2}\right)\right)$.

In this region (see Figure 3) one controls the weights of contours with boundary conditions $\psi_{2}$ and $\psi_{1}$. Therefore, one controls $\ln \Theta_{1}\left(\operatorname{Int}_{1} \Gamma^{2}\right)$, and

$$
\phi_{\Lambda}\left(\Gamma^{2}\right)=\exp [-\beta \mathcal{H}\left(\varphi_{\Gamma^{2}} \mid \psi_{2}\right)+\underbrace{\left.\ln \frac{\Theta_{1}\left(\operatorname{Int}_{1} \Gamma^{2}\right)}{\Theta_{2}\left(\operatorname{Int}_{1} \Gamma^{2}\right)}+\ln \frac{\Theta_{i\left(\Gamma^{2}\right)}\left(\Lambda\left(\Gamma^{2}\right)\right)}{\Theta_{i\left(\Gamma^{2}\right)}(\Lambda)}\right]}_{:=\mathrm{G}\left(\Gamma^{2}\right)} .
$$

By definition $z=\zeta+\mu^{*}$, so that (see (3.4))

$$
\begin{aligned}
-\beta \mathcal{H}\left(\varphi_{\Gamma^{2}} \mid \psi_{2}\right)(z) & +\mathrm{G}\left(\Gamma^{2}\right)(z)=-\beta \mathcal{H}\left(\varphi_{\Gamma^{2}} \mid \psi_{2}\right)\left(\mu^{*}\right)+\beta \Delta V\left(\Gamma^{2}\right) \zeta \\
& -\beta a\left(\varphi_{\Gamma^{2}}\right) \zeta+\int_{\mu^{*}}^{\mu^{*}+\zeta} \frac{d}{d z^{\prime}} \mathrm{G}\left(\Gamma^{2}\right)\left(z^{\prime}\right) d z^{\prime}+\mathrm{G}\left(\Gamma^{2}\right)\left(\mu^{*}\right) \\
& =-\beta \mathcal{H}\left(\varphi_{\Gamma^{2}} \mid \psi_{2}\right)\left(\mu^{*}\right)+\mathrm{G}\left(\Gamma^{2}\right)\left(\mu^{*}\right)+\beta \Delta V\left(\Gamma^{2}\right) \zeta \\
& +\underbrace{\int_{\mu^{*}}^{\mu^{*}+\zeta}\left(\frac{d}{d z^{\prime}} \mathrm{G}\left(\Gamma^{2}\right)\left(z^{\prime}\right)-\beta a\left(\varphi_{\Gamma^{2}}\right)\right) d z^{\prime}}_{:=\beta \Delta V\left(\Gamma^{2}\right) \mathrm{g}\left(\Gamma^{2}\right)(\zeta)} .
\end{aligned}
$$

This proves (4.12).

$$
\begin{aligned}
& \frac{d}{d \zeta} \mathrm{g}\left(\Gamma^{2}\right)(\zeta)=\frac{1}{\beta \Delta V\left(\Gamma^{2}\right)}( \frac{d}{d \zeta} \ln \Theta_{1}\left(\operatorname{Int}_{1} \Gamma^{2}\right)-\frac{d}{d \zeta} \ln \Theta_{2}\left(\operatorname{Int}_{1} \Gamma^{2}\right) \\
&\left.+\frac{d}{d \zeta} \ln \frac{\Theta_{i\left(\Gamma^{2}\right)}\left(\Lambda\left(\Gamma^{2}\right)\right)}{\Theta_{i\left(\Gamma^{2}\right)}(\Lambda)}-\beta a\left(\varphi_{\Gamma^{2}}\right)\right)
\end{aligned}
$$

The last term of the right-hand side of (4.28) is estimated using (3.5). The first two terms are estimated using Proposition 4.1 and Lemma 3.1. The third term is estimated by writing explicitly the logarithm of the quotient, using (3.10). After cancellation the resulting series is differentiated term by term and is estimated using the basic estimates of Proposition 4.1 and Lemma 3.1. For $\beta$ large enough,

$$
\left|\frac{d}{d \zeta} \mathrm{g}\left(\Gamma^{2}\right)(\zeta)\right| \leq C_{7} \mathrm{e}^{-\tau_{\star}(\beta)}+C_{8} \frac{\left|\Gamma^{2}\right|}{V\left(\Gamma^{2}\right)},
$$

for suitable constants $C_{7}$ and $C_{8}$. Moreover, there exists a constant $C_{9}$ so that

$$
\exp \left[-\beta\left\|\Gamma^{2}\right\|\left(1+C_{9} \delta\right)\right] \leq \phi_{\Lambda}\left(\Gamma^{2}\right)\left(\mu^{*}\right) \leq \exp \left[-\beta\left\|\Gamma^{2}\right\|\left(1-C_{9} \delta\right)\right] .
$$




\subsection{Proof of Lemma 4.4}

Proof of Lemma 4.4. One has

$$
-\left[u_{\Lambda}\left(\Gamma^{2}\right)\right]_{\mu^{*}}^{(k)}=\left[\phi_{\Lambda}\left(\Gamma^{2}\right)\right]_{\mu^{*}}^{(k)}+\left[\phi_{\Lambda}\left(\Gamma^{2}\right)\right]_{\mu^{*}}^{(k)} \sum_{n \geq 2} \frac{(-1)^{(n-1)}}{n} \frac{\left[\phi_{\Lambda}\left(\Gamma^{2}\right)^{n}\right]_{\mu^{*}}^{(k)}}{\left[\phi_{\Lambda}\left(\Gamma^{2}\right)\right]_{\mu^{*}}^{(k)}} .
$$

From (4.18) and (4.19) there exists a constant $C_{13}$,

$$
\frac{\left[\phi_{\Lambda}\left(\Gamma^{2}\right)^{n}\right]_{\mu^{*}}^{(k)}}{\left[\phi_{\Lambda}\left(\Gamma^{2}\right)\right]_{\mu^{*}}^{(k)}} \leq C_{13} \phi_{\Lambda}^{*}\left(\Gamma^{2}\right)^{(n-1)}\left(\frac{c_{+}}{c_{-}}\right)^{k} n^{k}
$$

The isoperimetric inequality $(4.5), R_{2}(n) \leq \chi_{2}(n) n^{-\frac{1}{d}}$ and the definition of $k$-large volume contour imply

$$
\beta\left\|\Gamma^{2}\right\| \geq \beta \chi_{2}\left(V\left(\Gamma^{2}\right)\right) V\left(\Gamma^{2}\right)^{\frac{d-1}{d}} \geq \hat{\theta} \beta R_{2}\left(V\left(\Gamma^{2}\right)\right) V\left(\Gamma^{2}\right) \geq k .
$$

Let $b:=C_{9} \delta$ (see (4.14)); one may assume $\frac{9}{10}-b \geq \frac{4}{5}$ by taking $\beta$ large enough. Then

$$
\begin{aligned}
\frac{c_{+}^{k}}{c_{-}^{k}} \sum_{n \geq 2} n^{k-1} \mathrm{e}^{-(n-1)(1-b) k} & \leq \frac{c_{+}^{k}}{c_{-}^{k}} \sum_{n \geq 2} \mathrm{e}^{-\frac{1}{10}(n-1) k} \mathrm{e}^{-k\left[\left(\frac{9}{10}-b\right)(n-1)-\ln n\right]} \\
& \leq \frac{c_{+}^{k}}{c_{-}^{k}} \sum_{n \geq 2} \mathrm{e}^{-\frac{1}{10}(n-1) k} \mathrm{e}^{-k\left[\frac{4}{5}(n-1)-\ln n\right]} \\
& \leq\left(\frac{c_{+}}{c_{-}} \mathrm{e}^{-\frac{1}{10}}\right)^{k} \sum_{n \geq 1} \mathrm{e}^{-\frac{1}{10} n k} \\
& \equiv\left(\frac{c_{+}}{c_{-}} \mathrm{e}^{-\frac{1}{10}}\right)^{k} D(k) .
\end{aligned}
$$

One chooses $A$ so small that $c_{+}(A) c_{-}(A)^{-1} \mathrm{e}^{-\frac{1}{10}} \leq 1$. Hence

$$
(1-D(k))\left[\phi_{\Lambda}\left(\Gamma^{2}\right)\right]_{\mu^{*}}^{(k)} \leq-\left[u_{\Lambda}\left(\Gamma^{2}\right)\right]_{\mu^{*}}^{(k)} .
$$

Using (4.19) one gets the result.

\section{Restoration of the analyticity of the pres- sure at first-order phase transition in the van der Waals limit}

This section is devoted to an exposition of Friedli's PhD thesis [Fr]. I shall treat in details the results, which are essential to understand the restoration 
of the analyticity of the pressure in the van der Waals limit: the coarsegrained description of the model and the analysis of the restricted phases. These two important topics are of great interest, independently of Mayer's conjecture. I also add new results about the constrained pressure $\widehat{p}_{\gamma}$ in subsection 5.6.

The Kac-Ising model with parameter $\gamma \in(0,1)$ is a ferromagnetic Ising model, whose range of interaction is $\gamma^{-1}$. Thus, the results of section 4 apply. There is a first order phase transition at zero magnetic field ${ }^{36}$ and large $\beta$. The pressure $p_{\gamma}(h, \beta)$ has no analytic continuation in $h$ at $h=0$ if $\beta \geq \beta_{\gamma}^{*}$. However, $\beta_{\gamma}^{*}$ tends to infinity as $\gamma$ tends to zero. In order to investigate the above question, it is necessary to find the existence of $\beta_{\star}$, independent of $\gamma$, so that for any $\beta \geq \beta_{\star}$ and any $\gamma \in(0,1)$ the pressure $p_{\gamma}(h, \beta)$ has no analytic continuation in $h$ at $h=0$. This is a difficult problem and the proof of the existence of $\beta_{\star}$ is a remarkable result by itself. It is necessary to study the model on a coarse-grained scale, related to the range $\gamma^{-1}$ of the interaction. The coarse-grained formulation of the model is based on a recent paper of Bovier and Zahradník [BoZ2], and is treated in subsections 5.2 and 5.3. One can re-formulate the model as a model of $\gamma$-dependent contours, so that Peierls' condition holds with a constant independent of $\gamma$. An essential difference with sections 3 and 4 is that the ground-state configurations are replaced now by the restricted phases. The study of these phases is a key step of the analysis. A polymer representation of the partition function of the restricted phases is derived in subsection 5.4, and the properties of these restricted phases are discussed in subsections 5.5 and 5.6 .

Once the coarse-grained description of the model is done, and the restricted phases are controlled, the proof of Mayer's conjecture follows the pattern of the proof of Theorem 4.1; it is technically more involved, except that by symmetry the phase transition point occurs at $h=0$ and there is no difference between contours with different labels. The starting point is to enumerate the contours and to define, as in point $\mathbf{I}$ of the proof of Theorem 4.1, the function $u_{\Lambda}(\Gamma)$. If $\Gamma_{1}$ is the first contour, then $\Theta_{i\left(\Gamma_{1}\right)}(\Lambda)$ is now the restricted partition function. This has the non-trivial effect of inducing interactions among contours. Another point, which should be emphasized, is the formulation of the variational problems (there is only one type of variational problems by symmetry) at point II of the proof of Theorem 4.1. A formulation of these problems as in [I2] would probably made the proof of Mayer's conjecture difficult, if not impossible. We refer the reader to $[\mathrm{Fr}]$ or $[\mathrm{FrPf} 2]$ for complete proofs.

\footnotetext{
${ }^{36}$ The parameter $\mu$ of section 3 is here the external magnetic field; I use the standard notation $h$ instead of $\mu$ in this section.
} 


\subsection{Kac-Ising model. Main results}

The model is a ferromagnetic Ising model with spin-variable $\sigma_{i}= \pm 1$ and interaction

$$
J_{\gamma}(x)=c_{\gamma} \gamma^{d} \varsigma(\gamma x)
$$

with $0<\gamma<1$, and $\varsigma: \mathbb{R}^{d} \rightarrow \mathbb{R}^{+}$a function whose support is the cube $[-1,+1]^{d}, \varsigma(x)=\varsigma(|x|)$, and such that

$$
\int \varsigma(x) \mathrm{d} x=1
$$

The constant $c_{\gamma}$ in the definition of the interaction is chosen so that

$$
\sum_{x \in \mathbb{Z}^{d}: x \neq 0} J_{\gamma}(x)=1
$$

The inverse of the scaling parameter $\gamma$ is the range of the interaction. Spin configurations are denoted in this section by $\sigma$ or $\eta$. $\Omega_{\Lambda}$ is the set of spin configurations in $\Lambda$ and $\Omega$ the set of spin configurations on $\mathbb{Z}^{d}$. The restriction of a configuration $\sigma$ to a subset $A \subset \mathbb{Z}^{d}$ is denoted by $\sigma_{A}$.

For a finite $\Lambda$ and $\sigma \in \Omega_{\Lambda}$, the Kac-Ising hamiltonian is

$$
H_{\Lambda}^{h}(\sigma):=-\sum_{\substack{\{i, j\} \subset \Lambda \\ i \neq j}} J_{\gamma}(i-j) \sigma_{i} \sigma_{j}-h \sum_{i \in \Lambda} \sigma_{i}, h \in \mathbb{R} .
$$

Let

$$
Z(\Lambda):=\sum_{\sigma \in \Omega_{\Lambda}} \exp \left(-\beta H_{\Lambda}^{h}(\sigma)\right)
$$

The pressure is

$$
p_{\gamma}(h):=\lim _{\Lambda \uparrow \mathbb{Z}^{d}} p_{\gamma, \Lambda}(h) \quad \text { with } \quad p_{\gamma, \Lambda}(h):=\frac{1}{\beta|\Lambda|} \ln Z(\Lambda) .
$$

The magnetization in $\Lambda$ is

$$
m_{\Lambda}(\sigma)=\frac{1}{|\Lambda|} \sum_{i \in \Lambda} \sigma_{i} \in[-1,+1] .
$$

The canonical partition function is

$$
Z(\Lambda, m):=\sum_{\substack{\sigma_{\Lambda} \in \Omega_{\Lambda}: \\ m_{\Lambda}(\sigma)=m}} \exp \left(-\beta H_{\Lambda}^{0}\left(\sigma_{\Lambda}\right)\right),
$$

and the free energy for given magnetization $m$

$$
f_{\gamma}(m):=-\lim _{\Lambda \uparrow \mathbb{Z}^{d}} \frac{1}{\beta|\Lambda|} \ln Z(\Lambda, m(\Lambda)) .
$$


In the definition of $f_{\gamma}$ the thermodynamic limit $\Lambda \uparrow \mathbb{Z}^{d}$ is taken along a sequence of cubes, and the sequence $m(\Lambda)$ is such that $m(\Lambda) \rightarrow m$.

There are few technical restrictions for the function $\varsigma$, which can be found in $[\mathrm{Fr}]$. I consider a specific and convenient choice,

$$
\varsigma(x):= \begin{cases}2^{-d} & \text { if } x \in[-1,1]^{d} ; \\ 0 & \text { otherwise. }\end{cases}
$$

In this setting, the main result for the free energy $f_{\gamma}$ is Theorem 2.3 which is re-called for convenience. Let

$$
m^{*}(\beta, \gamma)=\left.\frac{d}{d h} p_{\gamma}(h, \beta)\right|_{h=0+}>0 .
$$

Theorem 5.1. There exists $\beta_{\star}$ and $\gamma_{0}>0$ such that for all $\beta \geq \beta_{\star}$, $\gamma \in\left(0, \gamma_{0}\right), f_{\gamma}$ is analytic at any $m \in(-1,+1)$, except at $\pm m^{*}(\beta, \gamma)$.

$f_{\gamma}$ has no analytic continuation beyond $-m^{*}(\beta, \gamma)$ along the real path $m<$ $-m^{*}(\beta, \gamma)$.

$f_{\gamma}$ has no analytic continuation beyond $m^{*}(\beta, \gamma)$ along the real path $m>$ $m^{*}(\beta, \gamma)$.

The proof of Theorem 5.1 is obtained by working in the more appropriate grand canonical ensemble (in the lattice gas terminology), in which the constraint on the magnetization is controlled by a magnetic field. This means that one considers the pressure instead of the free energy, and then uses the fact that free energy and pressure are related by a Legendre transform,

$$
f_{\gamma}(m)=\sup _{h \in \mathbb{R}}\left(h m-p_{\gamma}(h)\right) .
$$

The analytic properties of $f_{\gamma}$ at $\pm m^{*}(\beta, \gamma)$ are obtained from those of $p_{\gamma}$ at $h=0$. By the Theorem of Lee and Yang $[\mathrm{LeY}], p_{\gamma}$ is analytic in the complex plane except possibly on the imaginary axis.

Theorem 5.2. There exists $\beta_{\star}, \gamma_{0}>0$ and a constant $C_{r}>0$ such that for all $\beta \geq \beta_{\star}, \gamma \in\left(0, \gamma_{0}\right)$, the following holds:

(1) The pressure $p_{\gamma}$ is $C^{\infty}$ at $0^{ \pm}$. There exists a constant $C_{+}>0$ such that for all $k \in \mathbb{N}$,

$$
\left|p_{\gamma}^{(k)}\left(0^{ \pm}\right)\right| \leq\left(C_{+} \gamma^{\frac{d}{d-1}} \beta^{-\frac{1}{d-1}}\right)^{k} k !^{\frac{d}{d-1}}+C_{r}^{k} k ! .
$$

(2) The pressure has no analytic continuation at $h=0$. More precisely, given $\beta$ and $\gamma$, there exists $C_{-}>0$, independent of $\beta$ and $\gamma$, and an unbounded increasing sequence of integers $k_{1}, k_{2}, \ldots$ such that for all $k \in$ $\left\{k_{1}, k_{2}, \ldots\right\}$,

$$
\left|p_{\gamma}^{(k)}\left(0^{ \pm}\right)\right| \geq\left(C_{-} \gamma^{\frac{d}{d-1}} \beta^{-\frac{1}{d-1}}\right)^{k} k !^{\frac{d}{d-1}}-C_{r}^{k} k ! .
$$

The integers $k_{i}$ depend on $\gamma$ and $\beta$, and

$$
\lim _{\gamma \rightarrow 0} k_{i}=+\infty
$$


Corollary 5.1. There exists a constant $C$ such that for small values of $k$, i.e. for $k \leq \gamma^{-d} \beta$, the following upper bound is true,

$$
\left|p_{\gamma}^{(k)}\left(0^{ \pm}\right)\right| \leq C^{k} k !
$$

Proof. Using Stirling's formula

$$
\left(\gamma^{\frac{d}{d-1}} \beta^{-\frac{1}{d-1}}\right)^{k} k !^{\frac{d}{d-1}}=\left(\gamma^{\frac{d}{d-1}} \beta^{-\frac{1}{d-1}}\right)^{k} k !^{\frac{1}{d-1}} k ! \sim\left(\gamma^{d} \beta^{-1} k \mathrm{e}^{-1}\right)^{\frac{k}{d-1}} k !
$$

Proof of Theorem 5.1. Using the symmetry $p_{\gamma}(h)=p_{\gamma}(-h)$,

$$
f_{\gamma}(m)=\sup _{h \geq 0}\left(h m-p_{\gamma}(h)\right) .
$$

By the Theorem of Lee and Yang, $h \mapsto p_{\gamma}(h)$ and $h \mapsto m_{\gamma}(h):=p_{\gamma}^{(1)}(h)$ are analytic in $\{\operatorname{Re} h>0\}$. Let $m^{*}=m^{*}(\beta, \gamma)$; for all $m \in\left(m^{*}, 1\right)$,

$$
f_{\gamma}(m)=h_{\gamma}(m) m-p_{\gamma}\left(h_{\gamma}(m)\right),
$$

where $h_{\gamma}(m)$ is the unique solution of the equation $m=m_{\gamma}(h)$. If $h \geq 0$, GKS inequalities imply that

$$
\begin{aligned}
p_{\gamma}^{(2)}(h) & =\beta \sum_{j \in \mathbb{Z}^{d}}\left\langle\sigma_{0} \sigma_{j}\right\rangle_{h}-\left\langle\sigma_{0}\right\rangle_{h}\left\langle\sigma_{j}\right\rangle_{h} \geq \beta\left(\left\langle\sigma_{0} \sigma_{0}\right\rangle_{h}-\left\langle\sigma_{0}\right\rangle_{h}\left\langle\sigma_{0}\right\rangle_{h}\right) \\
& =\beta\left(1-\left\langle\sigma_{0}\right\rangle_{h}^{2}\right) .
\end{aligned}
$$

Since $p_{\gamma}^{(2)}(h) \neq 0$ for all $h>0$, the Biholomorphic Mapping Theorem ${ }^{37}$ implies that $m \mapsto h_{\gamma}(m)$ is analytic in a complex neighbourhood of each $m \in\left(m^{*}, 1\right)$. So $f_{\gamma}$, which is a composition of analytic maps, is analytic on $\left(m^{*}, 1\right)$.

Proof that $f_{\gamma}$ has no analytic continuation at $m^{*}$. Assume this is wrong.

$$
h_{\gamma}^{(1)}\left(m^{*}\right)=\lim _{m \searrow m^{*}} h_{\gamma}^{(1)}(m)=\lim _{h \searrow 0} m_{\gamma}^{(1)}(h)^{-1}=\lim _{h \searrow 0} p_{\gamma}^{(2)}(h)^{-1} \neq 0,
$$

since $p_{\gamma}^{(2)}\left(0^{+}\right)$is bounded at $h=0$. Hence (5.1) implies that $h_{\gamma}=h_{\gamma}(m)$ can be inverted in a neighbourhood of $m^{*}$ and that the inverse, $m_{\gamma}=$ $m_{\gamma}(h)$, is analytic at $h=0$. This is a contradiction with Theorem 5.2.

\footnotetext{
${ }^{37}$ Let $g: D \rightarrow \mathbb{C}$ be an analytic function, $z_{0} \in D$ be a point such that $g^{\prime}\left(z_{0}\right) \neq 0$. Then there exists a domain $V \subset D$ containing $z_{0}$, such that the following holds: $V^{\prime}=g(V)$ is a domain, and the map $g: V \rightarrow V^{\prime}$ has an inverse $g^{-1}: V^{\prime} \rightarrow V$ which is analytic, and which satisfies, for all $\omega \in V^{\prime}, g^{-1^{\prime}}(\omega)=g^{\prime}\left(g^{-1}(\omega)\right)^{-1}$. The proof of this result can be found in [Rem1], pp. 281-282.
} 


\subsection{Coarse-grained description of the model}

In this subsection the model is formulated as a contour model with restricted phases. Let $N \geq 1$;

$$
B_{N}(x):=\left\{y \in \mathbb{Z}^{d}:|x-y| \leq N\right\} \quad \text { and } \quad B_{N}^{\bullet}(x):=B_{N}(x) \backslash\{x\} .
$$

The $N$-neighbourhood of $\Lambda \subset \mathbb{Z}^{d}$ is

$$
[\Lambda]_{N}:=\bigcup_{x \in \Lambda} B_{N}(x)
$$

If $\sigma_{\Lambda} \in \Omega_{\Lambda}, \eta_{\Lambda^{c}} \in \Omega_{\Lambda^{c}}$, the concatenation $\sigma_{\Lambda} \eta_{\Lambda^{c}} \in \Omega$ is by definition:

$$
\left(\sigma_{\Lambda} \eta_{\Lambda^{c}}\right)_{i}= \begin{cases}\left(\sigma_{\Lambda}\right)_{i} & \text { if } i \in \Lambda \\ \left(\eta_{\Lambda^{c}}\right)_{i} & \text { if } i \in \Lambda^{c}\end{cases}
$$

The interaction is rewritten as

$$
J_{\gamma}(x):=\left\{\begin{array}{cl}
\frac{1}{\left|B_{\gamma^{-1}}^{\bullet}(0)\right|} & \text { if } 0<|x| \leq \gamma^{-1} \\
0 & \text { otherwise. }
\end{array}\right.
$$

As in Pirogov-Sinai theory, the first step is to define the notion of a correct point, respectively incorrect point of a spin configuration. A point $i$ is $(\delta,+)$-correct for $\sigma$ if in its $\gamma^{-1}$-neighbourhood there are not too many spins with value -1 . The value of $\sigma_{i}$ itself does not matter.

Definition 5.1. Let $\delta \in(0,1), \sigma \in \Omega, i \in \mathbb{Z}^{d}$.

1. $i$ is $(\delta,+)$-correct for $\sigma$ if $\left|B_{\gamma^{-1}}^{\bullet}(i) \cap\left\{j: \sigma_{j}=-1\right\}\right| \leq \frac{\delta}{2}\left|B_{\gamma^{-1}}(i)\right|$.

2. $i$ is $(\delta,-)$-correct for $\sigma$ if $\left|B_{\gamma^{-1}}^{\bullet}(i) \cap\left\{j: \sigma_{j}=+1\right\}\right| \leq \frac{\delta}{2}\left|B_{\gamma^{-1}}(i)\right|$.

3. $i$ is $\delta$-correct for $\sigma$ if it is either $(\delta,+)$ - or $(\delta,-)$-correct for $\sigma$.

4. $i$ is $\delta$-incorrect for $\sigma$ if it is not $\delta$-correct.

If $\delta$ is sufficiently small, then in a $\gamma^{-1}$-neighbourhood of a $(\delta,+)$-correct point, all points are either $(\delta,+)$-correct or they are incorrect. So, $(\delta,+)$ correct points are distant from $(\delta,-)$-correct points.

Lemma 5.1. Let $\delta \in\left(0,2^{-d}\right), \sigma \in \Omega$. Then

(1) If $i$ is $(\delta,+)$-correct, the box $B_{\gamma^{-1}}(i)$ contains either $(\delta,+)$-correct or $\delta$-incorrect points.

(2) If $i$ is $(\delta,-)$-correct, the box $B_{\gamma^{-1}}(i)$ contains either $(\delta,-)$-correct or $\delta$-incorrect points. 
Proof. Let $i$ be $(\delta,+)$-correct for $\sigma$, and $j \in B_{\gamma^{-1}}(i)$. Clearly

$$
\left|B_{\gamma^{-1}}(i) \cap B_{\gamma^{-1}}(j)\right| \geq \frac{1}{2^{d}}\left|B_{\gamma^{-1}}(i)\right| .
$$

Therefore, there are at least

$$
\frac{1}{2^{d}}\left|B_{\gamma^{-1}}(i)\right|-\frac{\delta}{2}\left|B_{\gamma^{-1}}(i)\right| \geq \frac{1}{2} \frac{1}{2^{d}}\left|B_{\gamma^{-1}}(i)\right|=\frac{1}{2} \frac{1}{2^{d}}\left|B_{\gamma^{-1}}(j)\right|
$$

points, which are $(\delta,+)$-correct in $B_{\gamma^{-1}}(i) \cap B_{\gamma^{-1}}(j)$, i.e. $j$ cannot be $(\delta,-)$-correct.

From now on $\delta$ is fixed in $\left(0,2^{-d}\right)$. The cleaned configuration $\bar{\sigma} \in \Omega$ is defined by

$$
\bar{\sigma}_{i}:= \begin{cases}+1 & \text { if } i \text { is }(\delta,+) \text {-correct for } \sigma, \\ -1 & \text { if } i \text { is }(\delta,-) \text {-correct for } \sigma, \\ \sigma_{i} & \text { if } i \text { is } \delta \text {-incorrect for } \sigma .\end{cases}
$$

For any set $M \subset \mathbb{Z}^{d}$, the partial cleaning $\sigma_{M} \bar{\sigma}_{M^{c}}$ coincides with $\sigma$ on $M$ and with $\bar{\sigma}$ on $M^{c}$. The cleaning and partial cleaning are always done according to the original configuration $\sigma$, using the fixed parameter $\delta$.

The set of $\delta$-incorrect points of the configuration $\sigma$ is denoted by $I_{\delta}(\sigma)$. The important property of the cleaning operation is expressed in the next lemma.

Lemma 5.2. (I) Let $M \subset \mathbb{Z}^{d}$. If $i$ is $(\delta,+)$-correct for $\sigma$, then it remains $(\delta,+)$-correct for $\sigma_{M^{\prime}} \bar{\sigma}_{M^{c}}$. If $i$ is $(\delta,-)$-correct for $\sigma$, then it remains $(\delta,-)$ correct for $\sigma_{M} \bar{\sigma}_{M^{c}}$.

(II) Let $M_{1} \subset M_{2}, \delta^{\prime} \in(0, \delta]$. Then $I_{\delta^{\prime}}\left(\sigma_{M_{1}} \bar{\sigma}_{M_{1}^{c}}\right) \subset I_{\delta^{\prime}}\left(\sigma_{M_{2}} \bar{\sigma}_{M_{2}^{c}}\right)$.

Proof. (I) If $i$ is, say, $(\delta,+)$-correct for $\sigma$, then the cleaning of $\sigma$ has the only effect in the box $B_{\gamma^{-1}}(i)$ of changing some - spins into + spins (and never + spins into - spins $)$. This is a consequence of Lemma 5.1. Therefore $i$ remains $(\delta,+)$-correct for $\sigma_{M} \bar{\sigma}_{M^{c}}$.

(II) Assume that $i$ is a $\left(\delta^{\prime},+\right)$-correct point of $\sigma_{M_{2}} \bar{\sigma}_{M_{2}^{c}}$. One shows that it is also a $\left(\delta^{\prime},+\right)$-correct point of $\sigma_{M_{1}} \bar{\sigma}_{M_{1}^{c}}$. Since the configurations $\sigma_{M_{2}} \bar{\sigma}_{M_{2}^{c}}$ and $\sigma_{M_{1}} \bar{\sigma}_{M_{1}^{c}}$ differ only on $M_{2} \backslash M_{1}$, let $k \in B_{\gamma^{-1}}(i) \cap\left(M_{2} \backslash M_{1}\right)$. There are three possibilities for the spin at $k$.

(1) $k$ is $(\delta,+)$-correct for $\sigma$, and $\bar{\sigma}_{k}=+1$.

(2) $k$ is $\delta$-incorrect for $\sigma$, and $\bar{\sigma}_{k}=\sigma_{k}=\left(\sigma_{M_{2}} \bar{\sigma}_{M_{2}^{c}}\right)_{k}$.

(3) $k$ is $(\delta,-)$-correct for $\sigma$, and $\bar{\sigma}_{k}=-1$.

One excludes possibility (3), so that only (1) and (2) occur, which proves the lemma. Indeed, by (I), if $k$ is $(\delta,-)$-correct for $\sigma$, then $k$ is also $(\delta,-)$ correct for $\sigma_{M_{2}} \bar{\sigma}_{M_{2}^{c}}$. Therefore, by Lemma 5.1, $i$ is $(\delta,-)$-correct or $\delta$ incorrect for $\sigma_{M_{2}} \bar{\sigma}_{M_{2}^{c}}$. This contradicts the assumption that $i$ is $\left(\delta^{\prime},+\right)$ correct for $\sigma_{M_{2}} \bar{\sigma}_{M_{2}^{c}}$. 
I now turn to the definition of the contours. Let $C^{(l)}$ be a partition of $\mathbb{Z}^{d}$ made of disjoint cubes of side length $l \in \mathbb{N}, l=\nu \gamma^{-1}$ with $\nu>2$, and whose centers lie on the sites of a fixed sub-lattice of $\mathbb{Z}^{d}$. If $i \in \mathbb{Z}^{d}$, then $C_{i}^{(l)}$ is the unique element of the partition $C^{(l)}$, which contains the site $i$. The family of all subsets of $\mathbb{Z}^{d}$, which are unions of elements of $C^{(l)}$, is denoted by $\mathcal{L}^{(l)}$. For any set $A \subset \mathbb{Z}^{d}$, the thickening of $A$ is

$$
\{A\}_{l}:=\bigcup_{i \in A} C_{i}^{(l)} .
$$

As in Pirogov-Sinai theory, contours are defined by $\delta$-incorrect points. Since they are defined on the coarse-grained scale $l$, a tentative definition of the boundary of a configuration would be

$$
M^{\prime}=\left\{\left[I_{\delta}(\sigma)\right]_{\gamma^{-1}}\right\}_{l}
$$

Notice that any $j \notin M^{\prime}$ is either $(\delta,+)$-correct or $(\delta,-)$-correct. If the spin at $j \notin M^{\prime}$ is $(\delta,+)$-correct $((\delta,-)$-correct $)$, then after cleaning it is a +-spin (--spin). Moreover, by definition of correct/incorrect points, if $i \in M^{\prime}$ and if $j \notin M^{\prime}$ is $(\delta,+)$-correct, with $|i-j| \leq \gamma^{-1}$, then $i$ is also $(\delta,+)$-correct. Of course, after cleaning outside $M^{\prime}$, the above $i$ remains $(\delta,+)$-correct for the partially cleaned configuration. Unfortunately this is not strong enough, and one must require ${ }^{38}$ the stronger condition that $i$ is $(\tilde{\delta},+)$-correct for the partially cleaned configuration outside $M^{\prime}$, with $\tilde{\delta}<\delta$. Because of this new requirement the definition of boundary of a configuration is more delicate. To formulate this notion one introduces the family of subsets of $\mathcal{L}^{(l)}$ which have the desired properties, and then shows that this family of subsets is nonempty and stable for the intersection, so that one can define the boundary of a configuration as the smallest element of this family. The details are given in the next paragraph.

Let $\tilde{\delta} \in(0, \delta)$. For each $\sigma \in \Omega$ with $\left|I_{\tilde{\delta}}(\sigma)\right|<\infty$, let

$$
\mathcal{E}(\sigma):=\left\{M \in \mathcal{L}^{(l)}: M \supset\left[I_{\delta}(\sigma)\right]_{\gamma^{-1}}, M \supset\left[I_{\tilde{\delta}}\left(\sigma_{M^{\prime}} \bar{\sigma}_{M^{c}}\right)\right]_{\gamma^{-1}}\right\} .
$$

(1) $\mathcal{E}(\sigma)$ is not empty. Indeed, let $M_{0}:=\left\{\left[I_{\tilde{\delta}}(\sigma)\right]_{\gamma^{-1}}\right\}_{l}$. If $M_{0}=\emptyset$ then $I_{\tilde{\delta}}(\sigma)=I_{\delta}(\sigma)=\emptyset$ and any subset of $\mathbb{Z}^{d}$ is in $\mathcal{E}(\sigma)$. If $M_{0} \neq \emptyset$, then $M_{0} \in \mathcal{E}(\sigma)$, because $M_{0} \in \mathcal{L}^{(l)}, M_{0} \supset\left[I_{\tilde{\delta}}(\sigma)\right]_{\gamma^{-1}} \supset\left[I_{\delta}(\sigma)\right]_{\gamma^{-1}}$ and $M_{0} \supset$ $\left[I_{\tilde{\delta}}(\sigma)\right]_{\gamma^{-1}} \supset\left[I_{\tilde{\delta}}\left(\sigma_{M_{0}} \bar{\sigma}_{M_{0}^{c}}\right)\right]_{\gamma^{-1}}$ by Lemma 5.2 .

(2) $\mathcal{E}(\sigma)$ is stable by intersection. Indeed, let $A, B \in \mathcal{E}(\sigma)$. Then clearly $A \cap B \supset\left[I_{\delta}(\sigma)\right]_{\gamma^{-1}}$; moreover, by Lemma 5.2 ,

$$
\begin{aligned}
& A \supset\left[I_{\tilde{\delta}}\left(\sigma_{A} \bar{\sigma}_{A^{c}}\right)\right]_{\gamma^{-1}} \supset\left[I_{\tilde{\delta}}\left(\sigma_{A \cap B} \bar{\sigma}_{(A \cap B)^{c}}\right)\right]_{\gamma^{-1}}, \\
& B \supset\left[I_{\tilde{\delta}}\left(\sigma_{B} \bar{\sigma}_{B^{c}}\right)\right]_{\gamma^{-1}} \supset\left[I_{\tilde{\delta}}\left(\sigma_{A \cap B} \bar{\sigma}_{(A \cap B)^{c}}\right)\right]_{\gamma^{-1}} .
\end{aligned}
$$

${ }^{38}$ See proof of Theorem 5.3 
Hence, one defines the boundary of the configuration $\sigma$ as

$$
I^{*}(\sigma):=\bigcap_{M \in \mathcal{E}(\sigma)} M
$$

The next property of $I^{*}(\sigma)$ is essential to prove Peierls' condition: there are sufficiently many $\tilde{\delta}$-incorrect points in $I^{*}(\sigma)$ for the partially cleaned configuration $\sigma_{I^{*}} \bar{\sigma}_{I^{* c}}$.

Lemma 5.3. There exists, in the $2 \gamma^{-1}$-neighbourhood of each box $C^{(l)} \subset$ $I^{*}(\sigma)$, a point $j \in I^{*}(\sigma)$ which is $\tilde{\delta}$-incorrect for the configuration $\sigma_{I^{*}} \bar{\sigma}_{I^{* c}}$. Proof. Let $C^{(l)} \subset I^{*}(\sigma)$. First, suppose $I_{\delta}(\sigma) \cap\left[C^{(l)}\right]_{2 \gamma^{-1}} \neq \emptyset$. Then each $j \in I_{\delta}(\sigma) \cap\left[C^{(l)}\right]_{2 \gamma^{-1}}$ is $\delta$-incorrect for $\sigma$, and hence $\tilde{\delta}$-incorrect for $\sigma_{I^{*}} \bar{\sigma}_{I^{* c}}$, since $\tilde{\delta}<\delta$ and $\sigma$ and $\sigma_{I^{*}} \bar{\sigma}_{I^{* c}}$ coincide on $B_{\gamma^{-1}}(j)$.

Suppose $^{39}$ that $\left[I_{\delta}(\sigma)\right]_{\gamma^{-1}} \cap\left[C^{(l)}\right]_{\gamma^{-1}}=\emptyset$, and that the statement is wrong, i.e. $I_{\tilde{\delta}}\left(\sigma_{I^{*}} \bar{\sigma}_{I^{* c}}\right) \cap\left[C^{(l)}\right]_{2 \gamma^{-1}}=\emptyset$. Then, set $I^{\prime}:=I^{*} \backslash C^{(l)}$ and show that $I^{\prime} \in$ $\mathcal{E}(\sigma)$, a contradiction with the definition of $I^{*}$. First, $I^{\prime} \supset\left[I_{\delta}(\sigma)\right]_{\gamma^{-1}}$. Using Lemma $5.2, I^{*} \supset\left[I_{\tilde{\delta}}\left(\sigma_{I^{*}} \bar{\sigma}_{I^{* c}}\right)\right]_{\gamma^{-1}} \supset\left[I_{\tilde{\delta}}\left(\sigma_{I^{\prime}} \bar{\sigma}_{I^{\prime c}}\right)\right]_{\gamma^{-1}}$. Since $I_{\tilde{\delta}}\left(\sigma_{I^{*}} \bar{\sigma}_{I^{* c}}\right) \cap$ $\left[C^{(l)}\right]_{2 \gamma^{-1}}=\emptyset$ is equivalent to $\left[I_{\tilde{\delta}}\left(\sigma_{I^{*}} \bar{\sigma}_{I^{* c}}\right)\right]_{\gamma^{-1}} \cap\left[C^{(l)}\right]_{\gamma^{-1}}=\emptyset$, this implies that $I^{\prime} \supset\left[I_{\tilde{\delta}}\left(\sigma_{I^{\prime}} \bar{\sigma}_{I^{\prime c}}\right)\right]_{\gamma^{-1}}$, i.e. $I^{\prime} \in \mathcal{E}(\sigma)$.

Contrary to what happens in Pirogov-Sinai theory, it is less obvious to characterize the set of configurations which have the same boundary. Let

$$
\mathcal{A}(\sigma):=\left\{\sigma^{\prime}: \sigma_{I^{*}(\sigma)}^{\prime}=\sigma_{I^{*}(\sigma)}, I^{*}\left(\sigma^{\prime}\right)=I^{*}(\sigma)\right\} .
$$

Below, the symbol \# is used to denote either of the symbols + or - . Let $\Lambda^{\#}(\sigma)$ be the set of points of $I^{*}(\sigma)^{c}$ that are $(\delta, \#)$-correct for $\sigma$. By Lemma $5.1 d\left(\Lambda^{+}(\sigma), \Lambda^{-}(\sigma)\right)>l$, and $\mathbb{Z}^{d}$ is partitioned into

$$
\mathbb{Z}^{d}=I^{*}(\sigma) \cup \Lambda^{+}(\sigma) \cup \Lambda^{-}(\sigma) .
$$

Proposition 5.1. $\mathcal{A}(\sigma)=\mathcal{D}(\sigma)$ if

$\mathcal{D}(\sigma):=\left\{\sigma^{\prime}: \sigma_{I^{*}(\sigma)}^{\prime}=\sigma_{I^{*}(\sigma)}\right.$, each $i \in\left[\Lambda^{\#}(\sigma)\right]_{\gamma^{-1}}$ is $(\delta, \#)$-correct for $\left.\sigma^{\prime}\right\}$.

Proof. Let $I^{*}(\sigma) \neq \emptyset$ (otherwise the statement is obvious).

(1) Assume $\sigma^{\prime} \in \mathcal{A}(\sigma)$. Then $I^{*} \equiv I^{*}(\sigma)=I^{*}\left(\sigma^{\prime}\right) \supset\left[I_{\delta}\left(\sigma^{\prime}\right)\right]_{\gamma^{-1}}$, so that each $i \in\left[I^{* c}\right]_{\gamma^{-1}}$ is $\delta$-correct for $\sigma^{\prime}$. Let $A$ be a maximal connected component of $\left[I^{* c}\right]_{\gamma^{-1}}$. There exists $i \in A$ such that $i \in I^{*}$, since by assumption $I^{*} \neq \emptyset$. By Lemma 5.1, it suffices to show that $i$ is $(\delta,+)$ correct for $\sigma$ if and only if it is $(\delta,+)$-correct for $\sigma^{\prime}$. Assume this is not the case, e.g. suppose $i$ is $(\delta,+)$-correct for $\sigma$ and $(\delta,-)$-correct for $\sigma^{\prime}$, i.e

$$
\begin{aligned}
& \left|B_{\gamma^{-1}}^{\bullet}(i) \cap\left\{j:\left(\sigma_{I^{*}} \bar{\sigma}_{I^{* c}}\right)_{j}=-1\right\}\right| \leq \frac{\tilde{\delta}}{2}\left|B_{\gamma^{-1}}^{\bullet}(i)\right| \\
& \left|B_{\gamma^{-1}}^{\bullet}(i) \cap\left\{j:\left(\sigma_{I^{*}}^{\prime} \bar{\sigma}_{I^{* c}}^{\prime}\right)_{j}=+1\right\}\right| \leq \frac{\tilde{\delta}}{2}\left|B_{\gamma^{-1}}^{\bullet}(i)\right| .
\end{aligned}
$$

${ }^{39}$ Here I use the fact that $\left.A \cap[B]\right]_{2 \gamma^{-1}}=\emptyset$ if and only if $[A]_{\gamma^{-1}} \cap[B]_{\gamma^{-1}}=\emptyset$. 
Since $i \in I^{*}$,

$$
\left|B_{\gamma^{-1}}^{\bullet}(i) \cap I^{* c}\right| \leq\left(1-2^{-d}\right)\left|B_{\gamma^{-1}}^{\bullet}(i)\right|
$$

Since $\sigma_{I^{*}(\sigma)}^{\prime}=\sigma_{I^{*}(\sigma)}$, one gets a contradiction $\left(\tilde{\delta}<\delta<2^{-d}\right)$,

$$
\begin{aligned}
\left|B_{\gamma^{-1}}^{\bullet}(i)\right| & =\left|B_{\gamma^{-1}}^{\bullet}(i) \cap I^{* c}\right|+\left|B_{\gamma^{-1}}^{\bullet}(i) \cap\left\{j:\left(\sigma_{I^{*}} \bar{\sigma}_{I^{* c}}\right)_{j}=-1\right\} \cap I^{*}\right| \\
& +\left|B_{\gamma^{-1}}^{\bullet}(i) \cap\left\{j:\left(\sigma_{I^{*}}^{\prime} \bar{\sigma}_{I^{* c}}^{\prime}\right)_{j}=+1\right\} \cap I^{*}\right| \\
& \leq\left(1-2^{-d}+\tilde{\delta}\right)\left|B_{\gamma^{-1}}^{\bullet}(i)\right|<\left|B_{\gamma^{-1}}^{\bullet}(i)\right| .
\end{aligned}
$$

(2) Suppose $\sigma^{\prime} \in \mathcal{D}(\sigma)$. Since $\sigma^{\prime}$ coincides with $\sigma$ on $I^{*}(\sigma)$ and all points of $\left[I^{*}(\sigma)^{c}\right]_{\gamma^{-1}}$ are $\delta$-correct for $\sigma^{\prime}, I_{\delta}\left(\sigma^{\prime}\right)=I_{\delta}(\sigma)$. Thus $I^{*}(\sigma) \supset\left[I_{\delta}(\sigma)\right]_{\gamma^{-1}}=$ $\left[I_{\delta}\left(\sigma^{\prime}\right)\right]_{\gamma^{-1}}$. Then, since $\sigma_{I^{*}(\sigma)} \bar{\sigma}_{I^{*}(\sigma)^{c}}={\sigma^{\prime}}_{I^{*}(\sigma)}{\overline{\sigma^{\prime}}}_{I^{*}(\sigma)^{c}}$, one has

$$
I^{*}(\sigma) \supset\left[I_{\tilde{\delta}}\left(\sigma_{I^{*}(\sigma)} \bar{\sigma}_{I^{*}(\sigma)^{c}}\right)\right]_{\gamma^{-1}}=\left[I_{\tilde{\delta}}\left(\sigma_{I^{*}(\sigma)}^{\prime} \bar{\sigma}_{I^{*}(\sigma)^{c}}^{\prime}\right)\right]_{\gamma^{-1}} .
$$

Therefore $I^{*}(\sigma) \in \mathcal{E}\left(\sigma^{\prime}\right)$, i.e. $I^{*}\left(\sigma^{\prime}\right) \subset I^{*}(\sigma)$.

Assume $I^{*}(\sigma) \backslash I^{*}\left(\sigma^{\prime}\right) \neq \emptyset$. Using the fact that $\sigma$ and $\sigma^{\prime}$ coincide on $I^{*}(\sigma) \backslash I^{*}\left(\sigma^{\prime}\right)$, one has $\sigma_{I^{*}\left(\sigma^{\prime}\right)} \bar{\sigma}_{I^{*}\left(\sigma^{\prime}\right)^{c}}=\sigma_{I^{*}\left(\sigma^{\prime}\right)}^{\prime} \bar{\sigma}_{I^{*}\left(\sigma^{\prime}\right)^{c}}^{\prime}$. As above $I^{*}\left(\sigma^{\prime}\right) \supset$ $\left[I_{\tilde{\delta}}\left(\sigma^{\prime}{ }_{I^{*}\left(\sigma^{\prime}\right)}{\overline{\sigma^{\prime}}}_{\left.I^{*}\left(\sigma^{\prime}\right)^{c}\right)}\right)\right]_{\gamma^{-1}}=\left[I_{\tilde{\delta}}\left(\sigma_{I^{*}\left(\sigma^{\prime}\right)} \bar{\sigma}_{\left.I^{*}\left(\sigma^{\prime}\right)^{c}\right)}\right]_{\gamma^{-1}}\right.$. But $I^{*}\left(\sigma^{\prime}\right) \supset\left[I_{\delta}\left(\sigma^{\prime}\right)\right]_{\gamma^{-1}}$ $=\left[I_{\delta}(\sigma)\right]_{\gamma^{-1}}$, so that $I^{*}\left(\sigma^{\prime}\right) \in \mathcal{E}(\sigma)$, i.e. $I^{*}\left(\sigma^{\prime}\right) \supset I^{*}(\sigma)$, which contradicts the assumption. Therefore $I^{*}\left(\sigma^{\prime}\right)=I^{*}(\sigma)$ and $\sigma^{\prime} \in \mathcal{A}(\sigma)$.

Definition 5.2. The connected components of the boundary $I^{*}(\sigma)$ are the supports of the contours of the configuration $\sigma$. A connected component is written supp $\Gamma$. A contour is a couple $\Gamma=\left(\operatorname{supp} \Gamma, \sigma_{\Gamma}\right)$, where $\sigma_{\Gamma}$ is the restriction of $\sigma$ to $\operatorname{supp} \Gamma$.

The notions of label, external contour, interior of contour, compatibility of family of contours, boundary condition of a contour are defined in the same way as in section 3 . For each contour $\Gamma$ with boundary condition + , there exists a unique configuration $\sigma[\Gamma]$, which coincides with $\sigma_{\Gamma}$ on the supp $\Gamma$, and which is equal to the labels of the components of $\mathbb{Z}^{d} \backslash \operatorname{supp} \Gamma$ otherwise. I also denote, as before, supp $\Gamma$ by $\Gamma$ when no confusion arise; in particular $|\Gamma| \equiv|\operatorname{supp} \Gamma|$. Notice also that the distance between the supports of two different contours of the same configuration is at least $l$.

\subsection{Proof of Peierls' condition}

Let $\Lambda \in \mathcal{L}^{(l)}$ be a finite set, $\sigma_{\Lambda} \in \Omega_{\Lambda}$ and set $\sigma:=\sigma_{\Lambda}+{ }_{\Lambda^{c}}$. Let

$$
\phi_{i j}\left(\sigma_{i}, \sigma_{j}\right):=-\frac{1}{2} J_{\gamma}(i-j)\left(\sigma_{i} \sigma_{j}-1\right) \quad \text { and } \quad \phi_{i j}:=\phi_{i j}(+,-) \geq 0,
$$

and

$$
u\left(\sigma_{i}\right):=-h \sigma_{i} .
$$


The hamiltonian with boundary condition $+_{\Lambda^{c}}$ is

$$
H_{\Lambda}(\sigma):=H_{\Lambda}\left(\sigma_{\Lambda}+\Lambda^{c}\right)=\sum_{\substack{\{i, j\} \cap \Lambda \neq \emptyset \\ i \neq j}} \phi_{i j}\left(\sigma_{i}, \sigma_{j}\right)+\sum_{i \in \Lambda} u\left(\sigma_{i}\right) .
$$

One writes $I^{*}(\sigma)$ for $I^{*}(\sigma) \cap \Lambda$, and $\Lambda^{ \pm}(\sigma)$ for $\Lambda^{ \pm}(\sigma) \cap \Lambda$. The hamiltonian can be written in such a way that spins in regions $\Lambda^{\#}(\sigma)$ are subject to an effective external field $U^{\#}$.

The energy of the boundary of a configuration is by definition $H_{I^{*}}\left(\sigma_{I^{*}} \bar{\sigma}_{I^{* c}}\right)$, and

$$
H_{I^{*}}\left(\sigma_{I^{*}} \bar{\sigma}_{I^{* c}}\right)=\sum_{\Gamma}\left(\|\Gamma\|+\sum_{i \in \Gamma} u\left(\sigma[\Gamma]_{i}\right)\right),
$$

where the sum is over contours of the configuration $\sigma$, and

$$
\|\Gamma\|:=\sum_{\substack{\{i, j\} \cap \Gamma \neq \emptyset \\ i \neq j}} \phi_{i j}\left(\sigma[\Gamma]_{i}, \sigma[\Gamma]_{j}\right) .
$$

$\|\Gamma\|$ is the surface energy of $\Gamma$.

Let $h=0$ and $I^{*}=I^{*}(\sigma) . H_{\Lambda}(\sigma)-H_{I^{*}}\left(\sigma_{I^{*}} \bar{\sigma}_{I^{*} c}\right)$ is equal to

$$
\begin{aligned}
H_{\Lambda}(\sigma)-H_{I^{*}}\left(\sigma_{I^{*}} \bar{\sigma}_{I^{*} c}\right)=\sum_{\#}\left[\sum_{\{i, j\} \subset \Lambda^{\#}(\sigma)} \phi_{i j}\left(\sigma_{i}, \sigma_{j}\right)\right. \\
\left.+\sum_{\substack{i \in \Lambda^{\#}(\sigma) \\
j \in I^{*}}} \phi_{i j}\left(\sigma_{i}, \sigma_{j}\right)+\sum_{\substack{i \in \Lambda^{\#}(\sigma) \\
j \notin \Lambda}} \phi_{i j}\left(\sigma_{i},+\right)-\sum_{\substack{i \in \Lambda^{\#}(\sigma) \\
j \in I^{*}}} \phi_{i j}\left(\#, \sigma_{j}\right)\right] .
\end{aligned}
$$

Let $i \in \Lambda^{+}(\sigma)$. In the neighbourhood $B_{\gamma^{-1}}(i)$ of $i$, the majority of the spins are + -spins. If all the spins $\sigma_{j}=1, j \neq i$, and $\sigma_{i}=-1$, then this gives an effective field acting on the spin at $i$, which is equal to

$$
\sum_{j \in B_{\gamma^{-1}}^{\bullet}(i)} \phi_{i j}\left(\sigma_{i},+\right)
$$

For $i$ or $j \in \Lambda^{+}(\sigma)$, then one writes $\phi_{i j}\left(\sigma_{i}, \sigma_{j}\right)$ as

$$
\phi_{i j}\left(\sigma_{i}, \sigma_{j}\right) \equiv w_{i j}^{+}\left(\sigma_{i}, \sigma_{j}\right)+\phi_{i j}\left(\sigma_{i},+\right)+\phi_{i j}\left(+, \sigma_{j}\right),
$$

so that one can extract this effective magnetic field (Lemma 5.4). Notice that

$$
w_{i j}^{+}\left(\sigma_{i}, \sigma_{j}\right):=\phi_{i j}\left(\sigma_{i}, \sigma_{j}\right)-\phi_{i j}\left(\sigma_{i},+\right)-\phi_{i j}\left(+, \sigma_{j}\right)= \begin{cases}-2 \phi_{i j} & \text { if } \sigma_{i}=\sigma_{j}=-1 \\ 0 & \text { otherwise }\end{cases}
$$

Similarly, if $i$ or $j \in \Lambda^{-}(\sigma)$, then one defines

$$
w_{i j}^{-}\left(\sigma_{i}, \sigma_{j}\right):=\phi_{i j}\left(\sigma_{i}, \sigma_{j}\right)-\phi_{i j}\left(\sigma_{i},-\right)-\phi_{i j}\left(-, \sigma_{j}\right)= \begin{cases}-2 \phi_{i j} & \text { if } \sigma_{i}=\sigma_{j}=1 \\ 0 & \text { otherwise }\end{cases}
$$


Lemma 5.4. Define the potential

$$
U^{\#}\left(\sigma_{i}\right):=u\left(\sigma_{i}\right)+\sum_{j: j \neq i} \phi_{i j}\left(\sigma_{i}, \#\right)=-h \sigma_{i}+\sum_{j: j \neq i} \phi_{i j}\left(\sigma_{i}, \#\right) .
$$

Then

$$
H_{\Lambda}(\sigma)=H_{I^{*}}\left(\sigma_{I^{*}} \bar{\sigma}_{I^{* c}}\right)+\sum_{\#}\left(\sum_{\substack{\{i, j\} \cap \Lambda^{\#}(\sigma) \neq \emptyset \\ i \neq j}} w_{i j}^{\#}\left(\sigma_{i}, \sigma_{j}\right)+\sum_{i \in \Lambda^{\#}(\sigma)} U^{\#}\left(\sigma_{i}\right)\right) .
$$

The central result of this section is Proposition 5.2.

Proposition 5.2. The surface energy satisfies Peierls' condition, i.e. there exists $\rho=\rho(\tilde{\delta}, \nu)>0$ such that for all contours $\Gamma$,

$$
\|\Gamma\| \geq \rho|\Gamma| .
$$

The constant $\rho$ is independent of $\gamma$.

Remark. $|\Gamma|$ is the total number of lattice sites contained in the support of $\Gamma$. The support of a contour is a union of finitely many cubes of $\mathcal{L}^{(l)}$, each of side length $l=\nu \gamma^{-1}$. So

$$
|\Gamma| \geq\left(\nu \gamma^{-1}\right)^{d} \#\left\{C^{(l)} \subset \operatorname{supp} \Gamma\right\} .
$$

Another way of measuring the size of $\operatorname{supp} \Gamma$ would be to count the number of cubes $C^{(l)}$ contained in supp $\Gamma$. Hence Peierls' condition can be written

$$
\|\Gamma\| \geq \rho^{\prime} \gamma^{-d} \#\left\{C^{(l)} \subset \operatorname{supp} \Gamma\right\}
$$

and

$$
\mathrm{e}^{-\beta\|\Gamma\|} \leq \mathrm{e}^{-\beta \gamma^{-d} \rho^{\prime} \#\left\{C^{(l)} C \operatorname{supp} \Gamma\right\}} .
$$

The factor $\beta \gamma^{-d}$ can be interpreted as an effective temperature for the system on the coarse-grained scale $\gamma^{-1}$.

Proof. One first shows that the effective field acting on a spin $i$ is Lipschitz (with Lipschitz constant $2 \gamma$ ). Let $\sigma \in \Omega, i \in \mathbb{Z}^{d}, \# \in\{ \pm\}$. Define

$$
V_{\sigma}(i ; \#):=\sum_{j: j \neq i} \phi_{i j}\left(\#, \sigma_{j}\right) \text {. }
$$

Then, for $|x-y| \leq \gamma^{-1}$,

$$
\left|V_{\sigma}(x ; \#)-V_{\sigma}(y ; \#)\right| \leq \gamma|x-y| .
$$

Indeed,

$$
\begin{aligned}
V_{\sigma}(x ; \#) & -V_{\sigma}(y ; \#)=\sum_{\substack{j \in B_{\gamma^{-1}}(x) \\
j \notin B_{\gamma^{-1}}(y)}} \phi_{x j}\left(\#, \sigma_{j}\right) \\
& +\sum_{j \in B_{\gamma^{-1}}(x) \cap B_{\gamma^{-1}}(y)}\left(\phi_{x j}\left(\#, \sigma_{j}\right)-\phi_{y j}\left(\#, \sigma_{j}\right)\right)-\sum_{\substack{j \in B_{\gamma^{-1}}(y) \\
j \notin B_{\gamma}^{-1}(x)}} \phi_{y j}\left(\#, \sigma_{j}\right) .
\end{aligned}
$$


The second sum vanishes; the first (last) sum can be estimated for $|x-y| \leq$ $\gamma^{-1}$, by

$$
\sum_{\substack{j \in B_{\gamma^{-1}}(x) \\ j \notin B_{\gamma^{-1}}(y)}} \phi_{x j}\left(\#, \sigma_{j}\right) \leq \frac{\left|B_{\gamma^{-1}}(x)\right|-\left|B_{\gamma^{-1}}(x) \cap B_{\gamma^{-1}}(y)\right|}{\left|B_{\gamma^{-1}}^{\bullet}(0)\right|} \leq \frac{|x-y|}{2 \gamma^{-1}} .
$$

By Lemma 5.3 there exists in the $2 \gamma^{-1}$-neighbourhood of each $C^{(l)} \subset \Gamma$ a point $j \in \Gamma$ which is $\tilde{\delta}$-incorrect for $\sigma[\Gamma]$. Let $A$ be the set of all such points. One has $\Gamma \subset[A]_{l+2 \gamma^{-1}}$. Let $A_{0}$ be any $4 \gamma^{-1}$-approximant of $A$, that is $A_{0} \subset A$, two points of $A_{0}$ are at distance at least $4 \gamma^{-1}$, and $A \subset\left[A_{0}\right]_{4 \gamma^{-1}}$. Therefore $\Gamma \subset\left[A_{0}\right]_{l+6 \gamma^{-1}}$. This implies that

$$
|\Gamma| \leq\left|A_{0}\right|\left|B_{l+6 \gamma^{-1}}(0)\right| .
$$

Since each $i \in A_{0}$ is $\tilde{\delta}$-incorrect for $\sigma[\Gamma]$,

$$
\left|B_{\gamma^{-1}}^{\bullet}(i) \cap\left\{k: \sigma[\Gamma]_{k}=+1\right\}\right|>\frac{\tilde{\delta}}{2}\left|B_{\gamma^{-1}}(i)\right| \quad(i \text { is not }(\tilde{\delta},-) \text {-correct })
$$

and

$$
\left|B_{\gamma^{-1}}^{\bullet}(i) \cap\left\{k: \sigma[\Gamma]_{k}=-1\right\}\right|>\frac{\tilde{\delta}}{2}\left|B_{\gamma^{-1}}(i)\right| \quad(i \text { is not }(\tilde{\delta},+) \text {-correct }) .
$$

Hence, independently of the value of $\sigma_{i}$,

$$
V_{\sigma[\Gamma]}(i ;-)>\frac{\tilde{\delta}}{2} \quad \text { and } \quad V_{\sigma[\Gamma]}(i ;+)>\frac{\tilde{\delta}}{2}
$$

One has

$$
\begin{aligned}
\|\Gamma\| & \geq \frac{1}{2} \sum_{i \in A_{0}} \sum_{\substack{k \in B_{\gamma^{-1}}(i) \cap \Gamma \\
l: l \neq k}} \sum_{l \neq k} \phi_{k l}\left(\sigma[\Gamma]_{k}, \sigma[\Gamma]_{l}\right) \\
& =\frac{1}{2} \sum_{i \in A_{0}} \sum_{\substack{k \in B_{\gamma^{-1}}(i) \cap \Gamma\\
}} V_{\sigma[\Gamma]}\left(k ; \sigma[\Gamma]_{k}\right) \\
& \geq \frac{1}{2} \sum_{i \in A_{0}} \sum_{\substack{k \in B_{\gamma}-1(i) \cap C_{i}^{(l)} \\
|k-i| \leq \frac{\tilde{\delta}}{4} \gamma^{-1}}} V_{\sigma[\Gamma]}\left(k ; \sigma[\Gamma]_{k}\right) .
\end{aligned}
$$

Using (5.2) and (5.4),

$$
\begin{aligned}
V_{\sigma[\Gamma]}\left(k ; \sigma[\Gamma]_{k}\right) & =V_{\sigma[\Gamma]}\left(i ; \sigma[\Gamma]_{k}\right)+\left(V_{\sigma[\Gamma]}\left(k ; \sigma[\Gamma]_{k}\right)-V_{\sigma[\Gamma]}\left(i ; \sigma[\Gamma]_{k}\right)\right) \\
& \geq \frac{\tilde{\delta}}{2}-\gamma|k-i| \geq \frac{\tilde{\delta}}{4}
\end{aligned}
$$


From this one deduces the existence of $\rho>0$, independent of $\gamma \in\left(0, \gamma_{0}\right)$, such that (see $(5.3))$

$$
\|\Gamma\| \geq \frac{1}{2}\left|A_{0}\right| \frac{1}{2^{d}}\left|B_{\frac{\delta}{4} \gamma^{-1}}(0)\right| \frac{\tilde{\delta}}{4} \geq \frac{\tilde{\delta}}{2^{d+3}}\left|B_{l+6 \gamma^{-1}}(0)\right|^{-1}|\Gamma|\left|B_{\frac{\delta}{4} \gamma^{-1}}(0)\right| \geq \rho|\Gamma| .
$$

\subsection{Polymer representation of the partition function of the restricted phases}

The configurations of the restricted phases are those configurations such that either all points are $(\delta,+)$-correct or all points are $(\delta,-)$-correct. I consider the + case, the other case is similar. I first define the kind of boundary conditions, which are admissible for the restricted partition functions associated with these phases. Let $\Lambda$ be a finite subset in $\mathcal{L}^{(l)}$.

Definition 5.3. A boundary condition $\eta_{\Lambda^{c}} \in \Omega_{\Lambda^{c}}$ is + -admissible if each $i \in[\Lambda]_{\gamma^{-1}}$ is $(\tilde{\delta},+)$-correct for the configuration ${ }{ }_{\Lambda} \eta_{\Lambda^{c}}$, i.e. for each $i \in$ $[\Lambda]_{\gamma^{-1}}$,

$$
\left|B_{\gamma^{-1}}^{\bullet}(i) \cap B\left(\eta_{\Lambda^{c}}\right)\right| \leq \frac{\tilde{\delta}}{2}\left|B_{\gamma^{-1}}(i)\right|,
$$

where

$$
B\left(\eta_{\Lambda^{c}}\right):=\left\{j \in \Lambda^{c}:\left(\eta_{\Lambda^{c}}\right)_{j}=-1\right\} .
$$

Notice that the boundary condition specified by a contour on its interior is always admissible. Let $i \in[\Lambda]_{\gamma^{-1}}, \sigma_{\Lambda} \in \Omega_{\Lambda}$, and define

$$
1_{i}\left(\sigma_{\Lambda}\right):= \begin{cases}1 & \text { if } i \text { is }(\delta,+) \text {-correct for } \sigma_{\Lambda} \eta_{\Lambda^{c}} \\ 0 & \text { otherwise. }\end{cases}
$$

( $\eta_{\Lambda^{c}} \in \Omega_{\Lambda^{c}}$ is a +-admissible boundary condition.) The configurations which are allowed in a restricted phase are those verifying $1\left(\sigma_{\Lambda}\right)=1$, with

$$
1\left(\sigma_{\Lambda}\right):=\prod_{i \in[\Lambda]_{\gamma}-1} 1_{i}\left(\sigma_{\Lambda}\right) .
$$

Set $\sigma:=\sigma_{\Lambda} \eta_{\Lambda^{c}}$. The hamiltonian for the restricted system is the one obtained in Lemma 5.4 for a region of +-correct points. The restricted partition function with boundary condition $\eta_{\Lambda^{c}}$ is

$$
Z_{r}{ }^{+}\left(\Lambda ; \eta_{\Lambda^{c}}\right):=\sum_{\sigma_{\Lambda} \in \Omega_{\Lambda}} 1\left(\sigma_{\Lambda}\right) \exp \left(-\beta \sum_{\substack{\{i, j\} \cap \Lambda \neq \emptyset \\ i \neq j}} w_{i j}^{+}\left(\sigma_{i}, \sigma_{j}\right)-\beta \sum_{i \in \Lambda} U^{+}\left(\sigma_{i}\right)\right) .
$$

One shows that $Z_{r}{ }^{+}(\Lambda)$ can be written as

$$
Z_{r}^{+}(\Lambda)=e^{\beta h|\Lambda|} \mathcal{Z}_{r}^{+}(\Lambda),
$$


where $\mathcal{Z}_{r}^{+}(\Lambda)$ is the partition function of a polymer model, having a normally convergent cluster expansion in the domain

$$
H_{+}=\left\{h \in \mathbb{C}: \operatorname{Re} h>-\frac{1}{8}\right\} .
$$

The reason for $\ln Z_{r}{ }^{+}(\Lambda)$ to behave analytically at $h=0$ is that the presence of contours is suppressed by $1\left(\sigma_{\Lambda}\right)$, and that on each spin $\sigma_{i}=-1$ acts an effective magnetic field

$$
U^{+}(-1)=h+\sum_{j: j \neq i} \phi_{i j}=1+h,
$$

which is close to 1 when $h$ is in a neighbourhood of $h=0$.

I now explain how one can express the restricted partition function $Z_{r}{ }^{+}(\Lambda) \equiv Z_{r}{ }^{+}\left(\Lambda ; \eta_{\Lambda^{c}}\right)$ as the partition function of a polymer model.

The influence of a boundary condition can always be interpreted as a magnetic field acting on sites near the boundary. One rearranges the terms of the hamiltonian as follows:

$$
\sum_{\substack{\{i, j\} \subset \Lambda \\ i \neq j}} w_{i j}^{+}\left(\sigma_{i}, \sigma_{j}\right)+\sum_{i \in \Lambda}\left(U^{+}\left(\sigma_{i}\right)+\sum_{j \in \Lambda^{c}} w_{i j}^{+}\left(\sigma_{i},\left(\eta_{\Lambda^{c}}\right)_{j}\right)\right) .
$$

By defining a new effective non-homogeneous magnetic field

$$
\mu_{i}^{+}\left(\sigma_{i}\right):=U^{+}\left(\sigma_{i}\right)+h+\sum_{j \in \Lambda^{c}} w_{i j}^{+}\left(\sigma_{i},\left(\eta_{\Lambda^{c}}\right)_{j}\right)
$$

one can extract a volume term from $Z_{r}{ }^{+}(\Lambda)$ and get $Z_{r}{ }^{+}(\Lambda)=e^{\beta h|\Lambda|} \mathcal{Z}_{r}^{+}(\Lambda)$, where

$$
\mathcal{Z}_{r}^{+}(\Lambda):=\sum_{\sigma_{\Lambda} \in \Omega_{\Lambda}} 1\left(\sigma_{\Lambda}\right) \exp \left(-\beta \sum_{\substack{\{i, j\} \subset \Lambda \\ i \neq j}} w_{i j}^{+}\left(\sigma_{i}, \sigma_{j}\right)-\beta \sum_{i \in \Lambda} \mu_{i}^{+}\left(\sigma_{i}\right)\right) .
$$

Notice that the field $\mu_{i}^{+}\left(\sigma_{i}\right)$ becomes independent of $\eta_{\Lambda^{c}}$ when $d\left(i, \Lambda^{c}\right)>$ $\gamma^{-1}$. Since $w_{i j}^{+}\left(\sigma_{i}, \sigma_{j}\right)=0$, if $\sigma_{i}=+1$ or $\sigma_{j}=+1$, and $\mu_{i}^{+}(+1)=0$, only the spins $\sigma_{i}$ with $\sigma_{i}=-1$ interact. The location of these spins are identified with the vertices of a graph. For each vertex of this graph one has a factor $e^{-\beta \mu_{i}^{+}(-1)}$. When $h \in H_{+}$,

$$
\operatorname{Re} \mu_{i}^{+}(-1)=1+2 \operatorname{Re} h+\sum_{j \in \Lambda^{c}} w_{i j}^{+}\left(-,\left(\eta_{\Lambda^{c}}\right)_{j}\right) \geq 1-2 \frac{1}{8}-\tilde{\delta}>\frac{1}{2},
$$

since $\tilde{\delta}<2^{-d}$. The re-formulation of $\mathcal{Z}_{r}^{+}(\Lambda)$ in terms of polymers is done in three steps.

I. One expresses $\mathcal{Z}_{r}^{+}(\Lambda)$ as a sum over graphs, satisfying a certain constraint inherited from $1\left(\sigma_{\Lambda}\right)$. 
II. One associates to each graph a spanning tree and re-sum over all graphs having the same spanning tree. The weights of the trees have good decreasing properties (Lemma 5.5 and Corollary 5.2).

III. The constraint is expanded, yielding sets on which the constraint is violated. These sets are linked with trees. After a second partial re-summation, this yields a sum over polymers, which are nothing but particular graphs with vertices living on $\mathbb{Z}^{d}$ and whose edges are of length at most $\gamma^{-1}$.

I. Since in the restricted +-phase only the spins $\sigma_{j}=-1$ interact, to each configuration $\sigma$ one associates a graph $G_{\sigma}=\left(V_{\sigma}, E_{\sigma}\right)$, by defining

$V_{\sigma}:=\left\{j \in \Lambda: \sigma_{j}=-1\right\}$ and $E_{\sigma}:=\left\{e=\{i, j\}: i, j \in V_{\sigma},|i-j| \leq \gamma^{-1}\right\}$.

The energy of the configuration $\sigma$ can be written

$$
\sum_{e \in E_{\sigma}} w_{e}^{+}+\sum_{j \in V_{\sigma}} \mu_{j}^{+} \quad \text { with } w_{e}^{+}:=w_{i j}^{+}(-,-) \text {and } \mu_{j}^{+}:=\mu_{j}^{+}(-1) .
$$

Notice that $w_{e}^{+}=-2 \phi_{i j} \leq 0$. In the Boltzmann factor, if one writes

$$
\prod_{e \in E_{\sigma}} \mathrm{e}^{-\beta w_{e}^{+}}=\prod_{e \in E_{\sigma}}\left(\left(\mathrm{e}^{-\beta w_{e}^{+}}-1\right)+1\right)
$$

and expand the product, one obtains a sum over all partial graphs ${ }^{40}$ of $G_{\sigma}$. If $e=\{i, j\}$ is an edge of one of the partial graphs, then $|i-j| \leq \gamma^{-1}$. Therefore, let $\mathcal{G}_{\Lambda}$ be the family of simple non-oriented graphs $G=(V, E)$ where $V \subset \Lambda$ and each edge $e=\{i, j\} \in E$ has $|i-j| \leq \gamma^{-1}$. Expanding the product over edges leads to the following expression

$$
\mathcal{Z}_{r}^{+}(\Lambda)=\sum_{G \in \mathcal{G}_{\Lambda}} 1(V(G)) \prod_{e \in E(G)}\left(e^{-\beta w_{e}^{+}}-1\right) \prod_{i \in V(G)} e^{-\beta \mu_{i}^{+}},
$$

where $1(V):=1\left(\sigma_{\Lambda}(V)\right)$, and $\sigma_{\Lambda}(V) \in \Omega_{\Lambda}$ is defined by $\sigma_{\Lambda}(V)_{i}=-1$ if $i \in V,+1$ otherwise. In terms of graphs, the constraint $1(V(G))=1$ is satisfied if and only if

$$
\sum_{\substack{e=\{i, j\} \\ j \in V(G) \cup B}}\left|w_{e}^{+}\right| \leq \delta, \quad \forall i \in[\Lambda]_{\gamma^{-1}}, \quad \text { where } B:=B\left(\eta_{\Lambda^{c}}\right) .
$$

Moreover, the fact that the boundary condition $\eta_{\Lambda^{c}}$ is + -admissible means that

$$
\sum_{\substack{e=\{i, j\} \\ j \in B}}\left|w_{e}^{+}\right| \leq \tilde{\delta}, \quad \forall i \in[\Lambda]_{\gamma^{-1}}
$$

$\overline{{ }^{40} \text { A partial graph } G^{\prime} \text { of a graph }} G$ is a graph such that $V\left(G^{\prime}\right)=V(G)$ and $E\left(G^{\prime}\right) \subset$ $E(G)$. 
II. One chooses a deterministic algorithm ${ }^{41}$ that assigns to each connected graph $G_{0}$ a spanning tree $T\left(G_{0}\right)$, in a translation invariant way (that is if $G_{0}^{\prime}$ is obtained from $G_{0}$ by translation then $T\left(G_{0}^{\prime}\right)$ is obtained from $T\left(G_{0}\right)$ by the same translation). The algorithm is applied to each component of each graph $G$ appearing in the partition function. Let $\mathcal{T}_{\Lambda} \subset \mathcal{G}_{\Lambda}$ denote the set of all forests. Then

$$
\mathcal{Z}_{r}^{+}(\Lambda)=\sum_{T \in \mathcal{T}_{\Lambda}} 1(V(T)) \prod_{\mathrm{t} \in T} \omega^{+}(\mathrm{t})
$$

where the product is over trees of $T$, and the weight of each tree is defined by

$$
\omega^{+}(\mathrm{t}):=\sum_{\substack{G \in \mathcal{G}_{\Lambda}: \\ T(G)=\mathrm{t}}} \prod_{e \in E(G)}\left(e^{-\beta w_{e}^{+}}-1\right) \prod_{i \in V(G)} e^{-\beta \mu_{i}^{+}}
$$

Isolated sites $\{i\} \subset \Lambda$ are also considered as trees. In this case, $\omega^{+}(\{i\})=$ $e^{-\beta \mu_{i}^{+}}$.

Lemma 5.5. Let $T \in \mathcal{T}_{\Lambda}$ be a forest such that $1(V(T))=1$. Then, uniformly in $h \in H_{+}$, for each tree $\mathrm{t} \in T$,

$$
\left|\omega^{+}(\mathrm{t})\right| \leq \prod_{e \in E(\mathrm{t})}\left(e^{-\beta w_{e}^{+}}-1\right) \prod_{i \in V(\mathrm{t})} e^{-\frac{1}{4} \beta} .
$$

Proof. For each $\mathrm{t} \in T$, let $E^{*}(\mathrm{t})$ denote the set of edges of the maximal connected graph of $\left\{G \in \mathcal{G}_{\Lambda}: T(G)=\mathrm{t}\right\}$ (see [Pf1]). We can express the weight as follows:

$$
\begin{aligned}
\omega^{+}(\mathrm{t}) & =\prod_{e \in E(\mathrm{t})}\left(e^{-\beta w_{e}^{+}}-1\right) \prod_{i \in V(\mathrm{t})} e^{-\beta \mu_{i}^{+}} \sum_{\substack{G \in \mathcal{G}_{\Lambda}: \\
T(G)=\mathrm{t}}} \prod_{e \in E(G) \backslash E(\mathrm{t})}\left(e^{-\beta w_{e}^{+}}-1\right) \\
& =\prod_{e \in E(\mathrm{t})}\left(e^{-\beta w_{e}^{+}}-1\right) \prod_{i \in V(\mathrm{t})} e^{-\beta \mu_{i}^{+}} \prod_{e \in E^{*}(\mathrm{t}) \backslash E(\mathrm{t})} e^{-\beta w_{e}^{+}} .
\end{aligned}
$$

Since $1(V(T))=1$, the constraint (5.6) is satisfied, and the last product can be bounded by:

$$
\begin{aligned}
\prod_{e \in E^{*}(\mathrm{t}) \backslash E(\mathrm{t})} e^{\beta\left|w_{e}^{+}\right|} & \leq \prod_{i \in V(\mathrm{t})} \prod_{\substack{e=\{i, j\} \\
j \in V(\mathrm{t})}} e^{\beta\left|w_{e}^{+}\right|} \\
& =\prod_{i \in V(\mathrm{t})} \exp \left(\beta \sum_{\substack{e=\{i, j\} \\
j \in V(\mathrm{t})}}\left|w_{e}^{+}\right|\right) \leq \prod_{i \in V(\mathrm{t})} e^{\beta \delta} .
\end{aligned}
$$

This gives the result, since $\operatorname{Re} \mu_{i}^{+} \geq \frac{1}{2}$ by (5.5), and $\delta \leq 2^{-d} \leq \frac{1}{4}$.

\footnotetext{
${ }^{41}$ To be precise, one chooses the algorithm of chapter 3 of [Pf1].
} 
Notice that to obtain (5.9), one needs only that the bound

$$
\sum_{\substack{e=\{i, j\} \\ j \in V(\mathrm{t})}}\left|w_{e}^{+}\right| \leq \delta, \quad \forall i \in V(\mathrm{t}) .
$$

This is weaker than (5.6) and clearly $1(V(T))=1$ only if $(5.10)$ is satisfied for all $t \in T$. In the sequel we can thus assume that the trees we consider always satisfy (5.10), independently of each other. So the bound (5.8) can always be used. A direct consequence of the last lemma is the following result which shows that trees and their weights satisfy the main condition ensuring convergence of cluster expansions.

Corollary 5.2. Let $0<c \leq \frac{1}{8} \beta, \epsilon>0$. There exists $\gamma_{0}>0$ and $\beta_{1}=\beta_{1}(\epsilon)$ such that for all $\gamma \in\left(0, \gamma_{0}\right), \beta \geq \beta_{1}$, all $h \in H_{+}$, the following bound holds:

$$
\sum_{\mathrm{t}: V(\mathrm{t}) \ni 0}\left|\omega^{+}(\mathrm{t})\right| e^{c|V(\mathrm{t})|} \leq \epsilon .
$$

Proof. Using Lemma 5.5,

$$
\left|\omega^{+}(\mathrm{t})\right| e^{c|V(\mathrm{t})|} \leq \prod_{e \in E(\mathrm{t})}\left(e^{-\beta w_{e}^{+}}-1\right) \prod_{i \in V(\mathrm{t})} e^{-\frac{1}{8} \beta} .
$$

When $t$ is a single isolated point (the origin), then one has a factor $e^{-\frac{1}{8} \beta}$. When $V(\mathrm{t}) \ni 0, E(\mathrm{t}) \neq \emptyset$, one defines the generation of $\mathrm{t}$, gen $(\mathrm{t})$, as the number of edges of the longest self avoiding path in $t$ starting at the origin. The sum in (5.11) is bounded by (there are at least $g+1$ vertices in a tree $\mathrm{t}$ with $\operatorname{gen}(\mathrm{t})=g$ )

$$
\begin{aligned}
& e^{-\frac{1}{8} \beta}+\sum_{g \geq 1} \sum_{\substack{\mathrm{t}: V(\mathrm{t}) \ni 0 \\
\operatorname{gen}(\mathrm{t})=g}} \prod_{\substack{e \in E(\mathrm{t}) \\
\left(e^{-\beta w_{e}^{+}}-1\right)}} \prod_{i \in V(\mathrm{t})} e^{-\frac{1}{8} \beta} \\
& \leq e^{-\frac{1}{8} \beta}+\sum_{g \geq 1} e^{-\frac{1}{16} \beta g} \sum_{\substack{\mathrm{t}: V(\mathrm{t}) \ni 0 \\
\operatorname{gen}(\mathrm{t})=g}} \prod_{e \in E(\mathrm{t})}\left(e^{-\beta w_{e}^{+}}-1\right) \prod_{i \in V(\mathrm{t})} e^{-\frac{1}{16} \beta} \\
& \leq e^{-\frac{1}{8} \beta}+\sum_{g \geq 1} e^{-\frac{1}{16} \beta g} \alpha_{g},
\end{aligned}
$$

where $\left(V_{l}(\mathrm{t})\right.$ is the set of leaves of the tree $\left.\mathrm{t}\right)$ :

$$
\alpha_{g}:=\sum_{\substack{\mathrm{t}: V(\mathrm{t}) \ni 0 \\ \operatorname{gen}(\mathrm{t})=g}} \prod_{e \in E(\mathrm{t})}\left(e^{-\beta w_{e}^{+}}-1\right) \prod_{i \in V(\mathrm{t}) \backslash V_{l}(\mathrm{t})} e^{-\frac{1}{16} \beta} \prod_{i \in V_{l}(\mathrm{t})} e^{-\frac{1}{32} \beta} .
$$

One shows that $\alpha_{g+1} \leq \alpha_{g}$ for all $g \geq 1$. Let

$$
\gamma_{0}:=\sup \left\{\gamma>0: 2 c_{\gamma} \gamma^{d} \sup _{s} J(s) \leq \frac{1}{64}\right\} \text {. }
$$


Since $e^{-\beta w_{e}^{+}}-1 \leq \beta\left|w_{e}^{+}\right| e^{\beta\left|w_{e}^{+}\right|}$and $\left|w_{e}^{+}\right|=2 \phi_{i j}$, when $\gamma \leq \gamma_{0}$ one gets

$$
\sum_{e \ni 0}\left(e^{-\beta w_{e}^{+}}-1\right) e^{-\frac{1}{32} \beta} \leq \beta e^{-\frac{1}{64} \beta} \sum_{e \ni 0}\left|\omega_{e}^{+}\right| \leq 2 \beta e^{-\frac{1}{64} \beta} \equiv \beta \zeta(\beta) .
$$

Clearly, a tree $\mathrm{t}$ of generation $g+1$ can be obtained from a sub-tree $\mathrm{t}^{\prime} \subset \mathrm{t}$ of generation $g$ by attaching edges to leaves of $\mathrm{t}^{\prime}$. Let $x$ be a leaf of $\mathrm{t}^{\prime}$. The sum over all possible edges (if any) attached at $x$ is bounded by

$1+\sum_{k \geq 1} \frac{1}{k !} \sum_{e_{1} \ni x} \cdots \sum_{e_{k} \ni x} \prod_{i=1}^{k}\left(e^{-\beta w_{e_{i}}^{+}}-1\right) e^{-\frac{1}{32} \beta} \leq 1+\sum_{k \geq 1} \frac{1}{k !}(\beta \zeta(\beta))^{k}=e^{\beta \zeta(\beta)}$.

Assuming $\beta$ is large enough so that $\zeta(\beta) \leq \frac{1}{32}$, the weight of the leaf $x$ changes into $e^{-\frac{1}{16} \beta} e^{\beta \zeta(\beta)} \leq e^{-\frac{1}{32} \beta}$, which is exactly what appears in $\alpha_{g}$. This shows that $\alpha_{g+1} \leq \alpha_{g}$. Hence $\alpha_{g+1} \leq \alpha_{g} \leq \cdots \leq \alpha_{1}$, moreover, one shows as above that $\alpha_{1} \leq e^{-\frac{1}{32} \beta}$. This proves the result.

III. The constraint $1(V(T))$ depends on the relative positions of the trees. This "multi-body interaction" is treated by expanding

$$
\begin{aligned}
1(V(T)) & =\prod_{i \in[\Lambda]_{\gamma^{-1}}} 1_{i}(V(T))=\prod_{i \in[\Lambda]_{\gamma^{-1}}}\left(1+1_{i}^{c}(V(T))\right) \\
& =\sum_{M \subset[\Lambda]_{\gamma^{-1}}} \prod_{i \in M} 1_{i}^{c}(V(T))
\end{aligned}
$$

where $1_{i}^{c}(V(T)):=1_{i}(V(T))-1$. This yields

$$
\mathcal{Z}_{r}^{+}(\Lambda)=\sum_{T \in \mathcal{T}_{\Lambda}} \sum_{M \subset[\Lambda]_{\gamma}-1}\left(\prod_{i \in M} 1_{i}^{c}(V(T))\right)\left(\prod_{\mathrm{t} \in T} \omega^{+}(\mathrm{t})\right) .
$$

Consider a pair $(T, M)$. Let $i \in M$. The function $1_{i}^{c}(V(T))$ is non-zero only when $i$ is not $(\delta,+)$-correct; it depends on the presence of trees of $T$ in the $\gamma^{-1}$-neighbourhood of $i$ and possibly on the points of $B\left(\eta_{\Lambda^{c}}\right)$ if $B_{\gamma^{-1}}(i) \cap \Lambda^{c} \neq \emptyset$. To make this dependence only local, one links the $\gamma^{-1}$-neighbourhoods of the points of $M$ with the trees of $T$ as follows.

(1) Let $N=N(M)$ be the graph whose vertices are given by

$$
V(N):=\bigcup_{i \in M} B_{\gamma^{-1}}(i)
$$

There is an edge between two vertices of $N, x$ and $y$, if and only if $\langle x, y\rangle$ is a pair of nearest neighbours of the same box $B_{\gamma^{-1}}(i)$ for some $i \in M$. The graph $N$ decomposes naturally into connected components (in the sense of graph theory) $N_{1}, N_{2}, \ldots, N_{K}$. Some of these components can intersect $\Lambda^{c}$. 
(2) One links trees $\mathrm{t}_{i} \in T$ with components $N_{j} \in N$. To this end, one defines an abstract graph $\hat{G}$ : to each tree $\mathrm{t}_{i} \in T$, one associates a vertex $w_{i}$, and to each component $N_{j}$ one associates a vertex $z_{j}$. The edges of $\hat{G}$ are defined by the condition: $\hat{G}$ has only edges between vertices $w_{i}$ and $z_{j}$, and this occurs if and only if $V\left(\mathrm{t}_{i}\right) \cap V\left(N_{j}\right) \neq \emptyset$. Consider a connected component of $\hat{G}$, whose vertices $\left\{w_{i_{1}}, \ldots, w_{i_{l}}, z_{j_{1}}, \ldots, z_{j_{l}}\right\}$ correspond to a set $P_{l}^{\prime}=\left\{\mathrm{t}_{i_{1}}, \ldots, \mathrm{t}_{i_{l}}, N_{j_{1}}, \ldots, N_{j_{l}}\right\}$. One changes $P_{l}^{\prime}$ into a set $P_{l}$, using the following decimation procedure:

(a) if $P_{l}^{\prime}=\left\{\mathrm{t}_{i_{1}}\right\}$ is a single tree then $P_{l}:=P_{l}^{\prime}$.

(b) if $P_{l}^{\prime}$ is not a single tree, then

$\left(\mathrm{b}_{1}\right)$ delete from $P_{l}^{\prime}$ all trees $\mathrm{t}_{i_{k}}$ that have no edges,

$\left(\mathrm{b}_{2}\right)$ for all trees $\mathrm{t}_{i_{k}}$ containing at least one edge, delete all edges $e \in E\left(\mathrm{t}_{i_{k}}\right)$ whose both end-points lie in the same component $N_{j_{m}}$.

The resulting set is of the form $P_{l}=\left\{\mathrm{t}_{s_{1}}, \ldots, \mathrm{t}_{s_{l}}, N_{j_{1}}, \ldots, N_{j_{l}}\right\}$, where each tree $\mathrm{t}_{s_{i}}$ is a sub-tree of one of the trees $\left\{\mathrm{t}_{i_{1}}, \ldots, \mathrm{t}_{i_{l}}\right\} . P_{l}$ is called a polymer. The decimation procedure $P_{l}^{\prime} \Rightarrow P_{l}$ is depicted on Figure 4 .
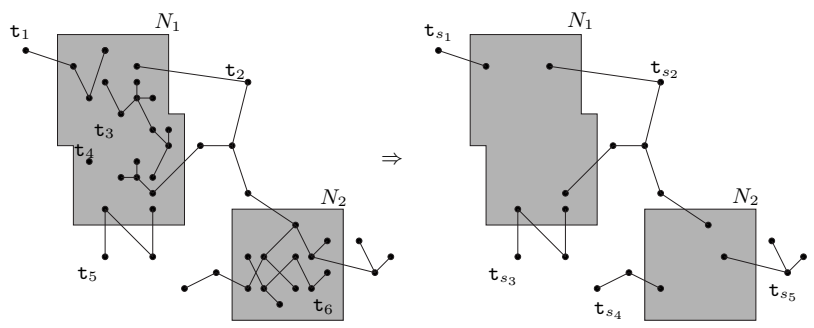

Figure 4: The decimation procedure $P_{l}^{\prime} \Rightarrow P_{l}$. The hatched polygons represent the body $\mathcal{B}\left(P_{l}\right)$ and the legs are the trees $\left\{\mathrm{t}_{s_{1}}, \mathrm{t}_{s_{2}}, \mathrm{t}_{s_{3}}, \mathrm{t}_{s_{4}}, \mathrm{t}_{s_{5}}\right\}$. Each $\mathrm{t}_{s_{j}}$ is a sub-tree of some $\mathrm{t}_{i}$.

The body of $P_{l}$ is

$$
\mathcal{B}\left(P_{l}\right):=V\left(N_{j_{1}}\right) \cup \cdots \cup V\left(N_{j_{l}}\right) ;
$$

the legs of $P_{l}$ are the trees $\left\{\mathrm{t}_{s_{1}}, \ldots, \mathrm{t}_{s_{l}}\right\}$. A polymer can have no body (in which case it is a tree of $\mathcal{T}_{\Lambda}$ ), or no legs (in which case it is a single component $N_{j_{1}}$ ). The support $V(P)$ is the total set of sites:

$$
V(P):=\bigcup_{\mathrm{t} \in \mathcal{L}(P)} V(\mathrm{t}) \cup \bigcup_{i} V\left(N_{i}\right) .
$$

Two polymers are compatible if and only if $V\left(P_{1}\right) \cap V\left(P_{2}\right)=\emptyset$, denoted $P_{1} \sim$ $P_{2}$. Therefore, to each pair $(T, M)$ there corresponds a family of pairwise 
compatible polymers $\{P\}:=\varphi(T, M)$. The set of all possible polymers constructed in this way is denoted by $\mathcal{P}_{\Lambda}^{+}\left(\eta_{\Lambda^{c}}\right)$. The representation of $\mathcal{Z}_{r}^{+}(\Lambda)$ in terms of polymers is then

$$
\mathcal{Z}_{r}^{+}(\Lambda) \equiv \mathcal{Z}_{r}\left(\mathcal{P}_{\Lambda}^{+}\left(\eta_{\Lambda^{c}}\right)\right)=\sum_{\substack{\{P\} \subset \mathcal{P}_{\Lambda}^{+}\left(\eta_{\Lambda^{c}}\right) \\ \text { compat. }}} \prod_{P \in\{P\}} \omega^{+}(P)
$$

where the weight is defined by

$$
\omega^{+}(P):=\sum_{\substack{(T, M): \\ \varphi(T, M)=P}}\left(\prod_{i \in M} 1_{i}^{c}(V(T))\right)\left(\prod_{\mathrm{t} \in T} \omega^{+}(\mathrm{t})\right) .
$$

Notice that the weight $\omega^{+}(P)$ depends on the position of $P$ inside the volume $\Lambda$, via the boundary condition $\eta_{\Lambda^{c}}$. This is the polymer representation of the restricted partition function.

\subsection{Properties of the restricted phases}

One can use now the cluster expansion method to study the restricted partition function.

Definition 5.4. The restricted pressures are defined by

$$
p_{r, \gamma}^{ \pm}:=\lim _{r \rightarrow \infty} \frac{1}{\beta\left|B_{r}(0)\right|} \ln Z_{r}{ }^{ \pm}\left(B_{r}(0) ; \pm_{B_{r}(0)^{c}}\right),
$$

where the thermodynamic limit is taken along a sequence of cubes.

Theorem 5.3. Let $\beta$ be large enough, $\gamma \in\left(0, \gamma_{0}\right), \Lambda \in \mathcal{L}^{(l)}$ and $\eta_{\Lambda^{c}}$ be a +-admissible boundary condition. Then

(1) The map $h \mapsto p_{r, \gamma}^{+}(h)$ is analytic in $\left\{\operatorname{Re} h>-\frac{1}{8}\right\}$.

(2) There exists a constant $C_{r}>0$ such that for all integers $k \geq 2$,

$$
\left|p_{r, \gamma}^{+(k)}(0)\right| \leq C_{r}^{k} k !
$$

Proof. There are two things to prove. First one establishes in Lemma 5.6 an upper bound for the weights $\omega^{+}(P)$ of the polymers ${ }^{42}$. Notice that the body of polymers can intersect $\Lambda^{c}$. At this point the condition $\delta-\tilde{\delta}>0$ is needed. Then one proves in Lemma 5.7 that the polymers satisfy the main criterion needed for having a convergent cluster expansion (see Lemma 3.2 ). Once this is done the proof of Theorem 5.3 is standard. I prove only Lemmas 5.6 and 5.7.

\footnotetext{
${ }^{42}$ The case $\omega^{-}(P)$ is similar by symmetry.
} 
Lemma 5.6. There exists $\beta_{2}$ and $\tau_{0}>0$ such that for all $\beta \geq \beta_{2}$, for all $\gamma \in\left(0, \gamma_{0}\right)$, and uniformly in $h \in H_{+}$the following holds: each polymer $P \in \mathcal{P}_{\Lambda}^{+}\left(\eta_{\Lambda^{c}}\right)$ satisfies

$$
\left|\omega^{+}(P)\right| \leq e^{-\tau_{0} \beta|\mathcal{B}(P)|} \prod_{e \in \mathcal{L}(P)}\left(e^{-\beta w_{e}^{+}}-1\right) \prod_{i \in \mathcal{L}(P)} e^{-\frac{1}{12} \beta} .
$$

Proof. If the body of the polymer $\mathcal{B}(P)=\emptyset$, then $P$ is a tree and the result follows from (5.8) of Lemma 5.5. Otherwise, $\left|\omega^{+}(P)\right|$ is bounded by

$$
\sum_{\substack{(T, M): \\ \varphi(T, M)=P}}\left(\prod_{i \in M}\left|1_{i}^{c}(V(T))\right|\right) \prod_{\mathrm{t} \in T}\left(\prod_{e \in E(\mathrm{t})}\left(e^{-\beta w_{e}^{+}}-1\right) \prod_{i \in V(\mathrm{t})} e^{-\frac{1}{4} \beta}\right) .
$$

Consider a pair $(T, M)$ such that $\varphi(T, M)=P$. Let $i_{0} \in M$, and assume $1_{i_{0}}^{c}(V(T)) \neq 0$. This implies, with regard to (5.6), that

$$
\sum_{\substack{e=\left\{i_{0}, j\right\} \\ j \in V(T) \cup B}}\left|w_{e}^{+}\right|>\delta .
$$

But, according to (5.7), one has

$$
\sum_{\substack{e=\left\{i_{0}, j\right\} \\ j \in B}}\left|w_{e}^{+}\right| \leq \tilde{\delta} .
$$

This implies the crucial lower bound

$$
\sum_{\substack{e=\left\{i_{0}, j\right\} \\ j \in V(T)}}\left|w_{e}^{+}\right| \geq \delta-\tilde{\delta}>0 .
$$

Since $\left|w_{e}^{+}\right|=2 \phi_{i j} \leq 2 c_{\gamma} \gamma^{d} \sup _{s} J(s)$, one can find a constant $c_{3}$ such that

$$
\left|V(T) \cap B_{\gamma^{-1}}^{\bullet}\left(i_{0}\right)\right|>(\delta-\tilde{\delta}) c_{3}\left|B_{\gamma^{-1}}\left(i_{0}\right)\right| .
$$

In this sense, the forests that contribute to $\omega^{+}(P)$ accumulate in the neighbourhood of each point $i_{0} \in M$. See Figure 5. Let $M_{0}$ be any $2 \gamma^{-1}$ approximant of $M$. Then one has $|\mathcal{B}(P)| \leq\left|M_{0}\right|\left|B_{3 \gamma^{-1}}(0)\right|$ and so

$$
|V(T) \cap \mathcal{B}(P)| \geq \sum_{i_{0} \in M_{0}}\left|V(T) \cap B_{\gamma^{-1}}\left(i_{0}\right)\right| \geq(\delta-\tilde{\delta}) c_{4}|\mathcal{B}(P)|,
$$

where $c_{4}$ is a constant. Now, each $i \in V(T)$ gets a factor $e^{-\frac{1}{4} \beta}=e^{-3 \frac{1}{12} \beta}$. One factor $e^{-\frac{1}{12} \beta}$ contributes to extract a term decreasing exponentially fast with the size of $\mathcal{B}(P)$, using (5.21):

$$
e^{-\frac{1}{12}(\delta-\tilde{\delta}) c_{4} \beta|\mathcal{B}(P)|} .
$$




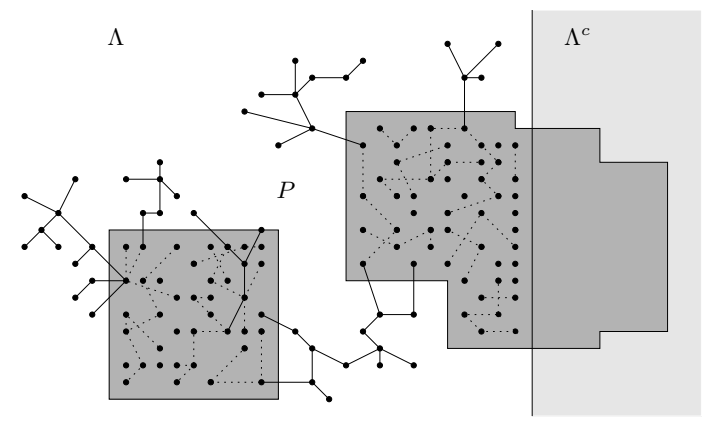

Figure 5: The re-summation of Lemma 5.6. The forest $T$ must have many points in $\mathcal{B}(P) \cap \Lambda$, as was shown in (5.21).

A second factor $e^{-\frac{1}{12} \beta}$ contributes to the weight of the legs. Extracting this contribution gives

$$
\prod_{e \in \mathcal{L}(P)}\left(e^{-\beta w_{e}^{+}}-1\right) \prod_{i \in \mathcal{L}(P)} e^{-\frac{1}{12} \beta},
$$

The last factor $e^{-\frac{1}{12} \beta}$ is used to re-sum over all the possible configurations of $T$ inside the body $\mathcal{B}(P)$ (see Figure 5), that is over all forests $T^{\prime}, V\left(T^{\prime}\right) \subset$ $\mathcal{B}(P)$, where each tree $\mathrm{t}^{\prime} \in T^{\prime}$ gets a weight bounded by

$$
\omega_{0}\left(\mathrm{t}^{\prime}\right):=\prod_{e \in E\left(\mathrm{t}^{\prime}\right)}\left(e^{-\beta w_{e}^{+}}-1\right) \prod_{i \in V\left(\mathrm{t}^{\prime}\right)} e^{-\frac{1}{12} \beta} .
$$

The remaining sum is thus bounded by

$$
\sum_{T^{\prime}: V\left(T^{\prime}\right) \subset \mathcal{B}(P)} \prod_{\mathrm{t}^{\prime} \in T^{\prime}} \omega_{0}\left(\mathrm{t}^{\prime}\right) \equiv \Theta_{0}(\mathcal{B}(P)) .
$$

This partition function can be studied with a convergent cluster expansion. Proceeding as for Corollary 5.2, one can take $\beta$ sufficiently large so that the weight $\omega_{0}\left(t^{\prime}\right)$ satisfies (5.11). One can then guarantee that

$$
\left|\log \Theta_{0}(\mathcal{B}(P))\right| \leq|\mathcal{B}(P)| .
$$

The sum over all possible sets $M$ such that $N(M)$ has a set of vertices given by $\mathcal{B}(P)$ is bounded by $2^{|\mathcal{B}(P)|}$. Altogether these bounds give

$$
e^{-\frac{1}{12}(\delta-\tilde{\delta}) c_{4} \beta|\mathcal{B}(P)|} 2^{|\mathcal{B}(P)|} e^{|\mathcal{B}(P)|} \equiv e^{-\tau_{0} \beta|\mathcal{B}(P)|},
$$

which finishes the proof. 
Lemma 5.7. Let $0<c \leq \min \left(\frac{\tau_{0}}{2}, \frac{1}{24}\right) \beta, \epsilon>0$. There exists $\beta_{3}=\beta_{3}(\epsilon)$, such that for all $\beta \geq \beta_{3}$ and for all $\gamma \in\left(0, \gamma_{0}\right)$, the following holds, uniformly in $h \in H_{+}$:

$$
\sum_{P: V(P) \ni 0}\left|\omega^{+}(P)\right| e^{c|V(P)|} \leq \epsilon .
$$

Proof. From Lemma 5.6

$$
\left|\omega^{+}(P)\right| \leq\left(\prod_{N \in P} \omega_{0}(N)\right)\left(\prod_{\mathrm{t} \in \mathcal{L}(P)} \omega_{0}(\mathrm{t})\right) \equiv \omega_{0}(P),
$$

where the weight of each component of the body $N$ is $\omega_{0}(N):=e^{-\tau_{0} \beta|V(N)|}$ and the weight of each leg $t$ is defined in (5.24). Fix $\epsilon>0$ small. It is easy to show that when $\beta$ is large enough,

$$
\sum_{N: V(N) \ni 0} \omega_{0}(N) e^{(c+\epsilon)|V(N)|} \leq \frac{1}{2} \epsilon,
$$

and, proceeding like in Corollary 5.2,

$$
\sum_{\mathrm{t}: V(\mathrm{t}) \ni 0} \omega_{0}(\mathrm{t}) e^{(c+\epsilon)|V(\mathrm{t})|} \leq \frac{1}{2} \epsilon .
$$

Let $n(P)$ denote the number of objects (components $N$ and trees t) contained in $P$. That is, if $P=\left\{\mathrm{t}_{1}, \ldots, \mathrm{t}_{L}, N_{1}, \ldots, N_{K}\right\}$, then $n(P)=L+K$. It is sufficient to show (by induction on $N=1,2, \ldots$ ) that

$$
\lambda_{N}:=\sum_{\substack{P: V(P) \ni 0 \\ n(P) \leq N}} \omega_{0}(P) e^{c|V(P)|} \leq \epsilon .
$$

If $N=1$ then $P$ can be either a single component $N$ or a tree t. The bound then follows from (5.29) and (5.30). Suppose $\beta$ is large and that the bound holds for $N$. If $P$ satisfies $V(P) \ni 0, n(P) \leq N+1$, one chooses an object of $P$ that contains the origin (which can be a tree $t_{0}$ or a component $N_{0}$ ), and decomposes $P$ as follows: either $P=\left\{N_{0}\right\} \cup\left\{P_{1}, \ldots, P_{k}\right\}$ with $V\left(N_{0}\right) \ni 0, V\left(P_{i}\right) \cap V\left(N_{0}\right) \neq \emptyset, n\left(P_{i}\right) \leq N, P_{i} \sim P_{j}$ for $i \neq j$, or $P=$ $\left\{\mathrm{t}_{0}\right\} \cup\left\{P_{1}, \ldots, P_{k}\right\}$ with $V\left(\mathrm{t}_{0}\right) \ni 0$, and $V\left(P_{i}\right) \cap V\left(\mathrm{t}_{0}\right) \neq \emptyset, n\left(P_{i}\right) \leq N$, $P_{i} \sim P_{j}$ for $i \neq j$. In the first case, one has, using the induction hypothesis and (5.29),

$$
\begin{aligned}
\sum_{N_{0}: V\left(N_{0}\right) \ni 0} \omega_{0}\left(N_{0}\right) e^{c\left|V\left(N_{0}\right)\right|} \sum_{k \geq 0} \frac{1}{k !}\left(\sum_{\substack{P: V(P) \cap V\left(N_{0}\right) \neq \emptyset \\
n(P) \leq N}} \omega_{0}(P) e^{c|V(P)|}\right)^{k} \\
\quad \leq \sum_{N_{0}: V\left(N_{0}\right) \ni 0} \omega_{0}\left(N_{0}\right) e^{c\left|V\left(N_{0}\right)\right|} \sum_{k \geq 0} \frac{1}{k !}\left(\left|V\left(N_{0}\right)\right| \lambda_{N}\right)^{k} \\
\quad \leq \sum_{N_{0}: V\left(N_{0}\right) \ni 0} \omega_{0}\left(N_{0}\right) e^{c\left|V\left(N_{0}\right)\right|} e^{\epsilon\left|V\left(N_{0}\right)\right|} \leq \frac{1}{2} \epsilon .
\end{aligned}
$$


In the second case the same computation yields, using (5.30),

$$
\begin{gathered}
\sum_{\mathrm{t}_{0}: V\left(\mathrm{t}_{0}\right) \ni 0} \omega_{0}\left(\mathrm{t}_{0}\right) e^{c\left|V\left(\mathrm{t}_{0}\right)\right|} \sum_{k \geq 0} \frac{1}{k !}\left(\sum_{\substack{P: V(P) \cap V\left(\mathrm{t}_{0}\right) \neq \emptyset \\
n(P) \leq N}} \omega_{0}(P) e^{c|V(P)|}\right)^{k} \\
\leq \sum_{\mathrm{t}_{0}: V\left(\mathrm{t}_{0}\right) \ni 0} \omega_{0}\left(\mathrm{t}_{0}\right) e^{c\left|V\left(\mathrm{t}_{0}\right)\right|} e^{\epsilon\left|V\left(\mathrm{t}_{0}\right)\right|} \leq \frac{1}{2} \epsilon .
\end{gathered}
$$

This shows that $\lambda_{N+1} \leq \epsilon$ and finishes the proof.

\subsection{Constrained pressure}

One can write the partition function $Z^{+}(\Lambda)$ as

$$
\mathrm{e}^{\beta h|\Lambda|} \mathcal{Z}_{r}^{+}(\Lambda) \Xi^{+}(\Lambda) .
$$

The term $\Xi^{+}(\Lambda)$ contains all contributions to the partition function, which come from the contours. This term is essential for the validity of Mayer's conjecture. One can express $\Xi^{+}(\Lambda)$ as the partition function of another polymer model, whose polymers are connected objects, which are made of contours and polymers describing the restricted phases. The entropy of these new polymers is controlled uniformly in the parameter $\gamma^{-1}$ (see $[\mathrm{Fr}]$ or [FrPf2]). Using (5.31) one can decompose the pressure $p_{\gamma}$ as

$$
p_{\gamma}(h, \beta)=p_{r, \gamma}^{+}(h, \beta)+\operatorname{sing}_{\gamma}^{+}(h, \beta) \quad \text { if } h \geq 0,
$$

where

$$
\operatorname{sing}_{\gamma}^{+}(h, \beta):=\lim _{n \rightarrow \infty} \frac{1}{\beta\left|B_{n}(0)\right|} \ln \Xi^{+}\left(B_{n}(0)\right) .
$$

A similar decomposition holds for $h \leq 0$. The term $\operatorname{sing}_{\gamma}^{+}(h)$ is the contribution to the pressure, which is due to the presence of contours. The control of this term is not easy, but it can be done in a fixed neighborhood of $h=0$, more precisely, there exist $h^{*}>0$ and two positive constants $a$ and $b$, such that

$$
\left|\operatorname{sing}_{\gamma}^{+}(h, \beta)\right| \leq a \mathrm{e}^{-b \beta \gamma^{-d}} \text { for all } h, 0 \leq h \leq h^{*} .
$$

The origin of the term $\mathrm{e}^{-b \beta \gamma^{-d}}$ is due to the Peierls' condition (see Proposition 5.2 and the remark following it). The main reason why this term is controlled only for $0 \leq h \leq h^{*}$ is that one controls simultaneously both restricted phases, which mediate interactions among the contours of the model, only in a neighborhood of $h=0[\mathrm{Fr}]$. As already mentioned in subsection 2.4 , it is the term $\operatorname{sing}_{\gamma}^{+}$which is responsible for the absence of an analytic continuation of $p_{\gamma}$ at $h=0$. Indeed, it is the presence at the phase transition point of stable droplets of the --phase of arbitrary size which is responsible for the absence of an analytic continuation of the pressure. 
The constrained pressure $\widehat{p}_{\gamma}$ discussed in subsection 2.4 is defined as follows. Let $Z_{\delta}^{\text {per }}\left(B_{n}(0)\right)$ be the partition function of the model in the box $B_{n}(0)$, with periodic boundary conditions and configurations having only $\delta$-correct points. It follows from Lemma 5.1 that

$$
Z_{\delta}^{\text {per }}\left(B_{n}(0)\right)=Z_{\delta,+}^{\text {per }}\left(B_{n}(0)\right)+Z_{\delta,-}^{\text {per }}\left(B_{n}(0)\right),
$$

where $Z_{\delta,+}^{\text {per }}\left(B_{n}(0)\right)$, resp. $Z_{\delta,-}^{\text {per }}\left(B_{n}(0)\right)$, is the contribution to $Z_{\delta}^{\text {per }}\left(B_{n}(0)\right)$ of the configurations having only $(\delta,+)$-correct points, respectively $(\delta,-)$ correct points. By definition

$$
\widehat{p}_{\gamma}(h, \beta):=\lim _{n \rightarrow \infty} \frac{1}{\beta\left|B_{n}(0)\right|} \ln Z_{\delta}^{\mathrm{per}}\left(B_{n}(0)\right) .
$$

By Hölder's inequality the constrained pressure $\widehat{p}_{\gamma}(h, \beta)$ is convex in $h$; it is symmetric in $h$. Let $h>0$. Then

$$
Z_{\delta}^{\text {per }}\left(B_{n}(0)\right)=Z_{\delta,+}^{\text {per }}\left(B_{n}(0)\right)\left(1+\frac{Z_{\delta,-}^{\text {per }}\left(B_{n}(0)\right)}{Z_{\delta,+}^{\text {per }}\left(B_{n}(0)\right)}\right) .
$$

In the numerator of the above fraction the majority of spins have value -1 , so that the fraction has a negligible contribution in the thermodynamic limit. Therefore

$$
\widehat{p}_{\gamma}(h, \beta)= \begin{cases}p_{r, \gamma}^{+}(h, \beta) & \text { if } h \geq 0 \\ p_{r, \gamma}^{-}(h, \beta) & \text { if } h \leq 0 .\end{cases}
$$

The constrained pressure $\widehat{p}_{\gamma}$ has a phase transition point at $h=0$ (for large $\beta)$, since

$$
\widehat{m}_{\gamma}^{*}(\beta):=\left.\frac{d}{d h} \widehat{p}_{\gamma}(h, \beta)\right|_{h=0+}=\left.\frac{d}{d h} p_{r, \gamma}^{+}(h, \beta)\right|_{h=0}>0 .
$$

From Theorem 5.3 one concludes that $\widehat{p}_{\gamma}$ has an analytic continuation $\tilde{p}_{\gamma}^{+}=p_{r, \gamma}^{+}$from $h>0$ to $h>-1 / 8$. On $H_{+}$the family of analytic functions $\left\{p_{r, \gamma}^{+}\right\}$is a normal family, because these functions are uniformly bounded on compact sets. This is a consequence of Lemmas 5.6 and 5.7, since one controls the cluster expansion uniformly in $\gamma$. In the van der Waals limit one can identify the constrained pressure with the mean-field pressure. Indeed, from (5.33),

$$
\lim _{\gamma \rightarrow 0} \widehat{p}_{\gamma}^{+}(h, \beta)=\lim _{\gamma \rightarrow 0} p_{\gamma}(h, \beta)=p_{\mathrm{mf}}(h, \beta) \quad \text { if } 0<h<h^{*} .
$$

The last equality is proved in $[\mathrm{Pr}]$. By Vitali's Theorem one has convergence for all $h \in H_{+}$(locally uniformly). By Weierstrass' Theorem the same is true for the derivatives of the constrained pressure. Hence

$$
\lim _{\gamma \rightarrow 0} \widehat{p}_{\gamma}(h, \beta)=p_{\mathrm{mf}}(h, \beta) \quad \text { and } \quad \lim _{\gamma \rightarrow 0} \widehat{m}_{\gamma}^{*}(\beta)=m^{*}(\beta) .
$$




\section{Concluding remarks}

Mayer's conjecture is proved for lattice models with two ground-states verifying Peierls' condition, at low enough temperature. The whole proof shows clearly the role of the phase separation phenomenon and from the study of the restoration of an analytic continuation of the pressure in the van der Waals limit one gets new understanding of models with weak and long-range interactions. There are still difficult and essentially totally open problems. I shall briefly mention three of them.

One does not know much about the nature of the singularity of the pressure at the transition point. Related to this problem is the question of a possible analytic continuation of the pressure along a path in the complex $z$-plane. In [F] Fisher proved that a droplet model may have such an analytic continuation ${ }^{43}$. However, one should be aware that existence or not of an analytic continuation is a very delicate question, as I have already shown in this paper. It is possible to define another version of the

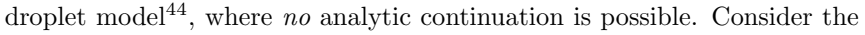
function $f$ defined on $\{h \in \mathbb{C}:$ Reh $\geq 0\}$ by the series

$$
f(z):=\sum_{n=0}^{\infty} \exp \left(-\lambda n^{d-1}-h n^{d}\right) \quad \text { where } \lambda>0 \text { and } d \geq 2 .
$$

The droplets are here cubic droplets of linear sizes $n$. This series is a so-called lacunary series ${ }^{45}$, i.e. of the form

$$
\sum_{m=1}^{\infty} a_{m} z^{k(m)} \quad \text { where } \quad z=\mathrm{e}^{-h}
$$

with

$$
k(m+1)-k(m)=(m+1)^{d}-m^{d} \rightarrow \infty \quad \text { if } \quad m \rightarrow \infty .
$$

This series, as a consequence of a general theorem of complex analysis ${ }^{46}$, Fabry's Theorem [Rem2], has $\{z \in \mathbb{C}:|z|=1\}$ as its natural boundary. Hence $f$, as a function of $h$, cannot be analytically continued from $\{h \in$ $\mathbb{C}:$ Reh $>0\}$ across Reh $=0$. For a brief discussion of these questions see [P] p.274.

Another already mentioned problem is Mayer's conjecture for real gases, i.e. in the continuum.

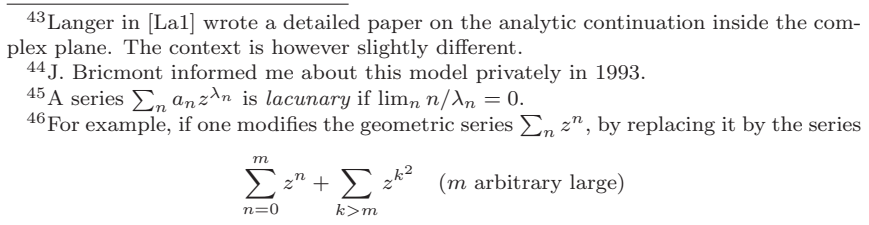

then this series has the boundary of the unit disc as natural boundary. 
The first two problems are mathematically well-defined. This is not the case of the next one, which is from my viewpoint very interesting, and which has been touched only a little in this paper. The very successful Cahn-Hilliard theory is based on free energy functionals, which are nonconvex below the critical temperature, and which are usually treated as given semi-phenomenological quantities. Is it possible to derive such free energy functionals in some controlled way, starting from the basic principles of Statistical Mechanics? 


\section{Bibliography}

[A] A.F. Andreev, Singularity for Thermodynamic Quantities at a First Order Phase Transition Soviet Physics JETP 18, 14151416 (1964).

[An] T. Andrews, On the Gaseous and Liquid States of Matter, Phil. Trans. Roy. Soc. 159, 575-590 (1869).

[Ban] W. Band, Dissociation Treatment of Condensing Systems $J$. Chem. Phys. 7, 324-326 (1939).

[Ba] G.A. Baker, Jr, One-dimensional order-disorder model which approaches phase transition, Phys. Rev. 122, 1477-1484 (1961).

[Bij] A. Bijl, Discontinuities in the Energy and Specific Heat, Dissertation, Leiden (1938).

[dB1] J. De Boer, Théorie de la condensation, Extrait des Comptes Rendus de la 2e Réunion de Chimie Physique 8-17 (1952).

[dB2] J. De Boer, van der Waals in his time and the present revival, Physica 73, 1-27 (1974).

[BorIm] C. Borgs, J.Z. Imbrie, A Unified Approach to Phase Diagrams in Field Theory and Statistical Mechanics, Commun. Math. Phys. 123, 305-328 (1989).

[BorKo] C. Borgs, R. Kotecký, Surface-induced finite-size effects for firstorder phase transitions. J. Stat. Phys. 79, 43-115 (1995).

[B] M. Born, The Statistical Mechanics of Condensing Systems, Physica 4, 1034-1044 (1937).

[BF] M. Born, K. Fuchs, The statistical mechanics of condensing systems, Proc. Royal Soc. A166, 391-414 (1938).

[BoZ1] A. Bovier, M. Zahradník, The low-temperature phases of the Kac-Ising models, J. Stat. Phys. 87, 311-332 (1997). 
[BoZ2] A. Bovier, M. Zahradník, Pirogov-Sinai Theory for Long Range Spin Systems, Mark. Proc. Rel. Fields 8, 443-478, (2002).

[Bro] R. Brout, Statistical Mechanical Theory of ferromagnetism. High Density Behavior, Phys. Rev. 118, 1009-1019 (1960).

[CHi] J.W. Cahn, J.E. Hilliard, Free Energy of a Nonuniform System. I. Interfacial free Energy, J. Chem. Phys. 28, 258-267 (1958).

[CPr] M. Cassandro, E. Presutti, Phase transitions in Ising systems with long but finite range interactions, Mark. Proc. Rel. Fields 2, 241-262 (1996).

[Cl] R. Clausius, Über einen auf die Wärme anwendbaren mechanischen Satz, Sitzungsberichte der Niederrheinischen Gesellschaft, Bonn, 114-119 (1870). English translation in Philosophical Magazine 40, 122-127 (1870).

[Ca] H.B. Callen, Thermodynamics and an Introduction to Thermostatistics, second edition, John Wiley, New-York (1985).

[CoM $]$ C. Coulon, S. Moreau, Physique Statistique et Thermodynamique, Dunod, Paris (2000).

[D] M. Dresden, Kramers's Contributions to Statistical Mechanics, Physics Today, September, 26-33 (1988).

[ELi] G. Emch, C. Liu, The Logic of Thermostatistical Physics, Springer, Berlin (2002).

[F] M.E. Fisher, The Theory of Condensation and the Critical Point, Physics 3, 255-283, (1967).

[Fre1] J. Frenkel, Statistical Theory of Condensation Phenomena $J$. Chem. Phys. 7, 200-201 (1939).

[Fre2] J. Frenkel, A General Theory of Heterophase Fluctuations and Pretransition Phenomena J. Chem. Phys 7, 538-547 (1939).

[Fr] S. Friedli, On the non-analytic behaviour of thermodynamic potential at first order phase transitions $\mathrm{PhD}$-thesis 2784, Ecole Polytechnique Fédérale, Lausanne (2003). Available at http://library.epfl.ch/theses/

[FrPf1] S. Friedli, C.-E. Pfister, On the Singularity of the Free Energy at First Order Phase Transition, Commun. Math. Phys. 245, 69-103 (2004).

[FrPf2] S. Friedli, C.-E. Pfister, Non-Analyticity and the van der Waals limit, J. Stat. Phys. 114, 665-734 (2004). 
[FrPf3] S. Friedli, C.-E. Pfister, Rigorous Analysis of Singularities and Absence of Analytic Continuation at First Order Phase Transition Points in Lattice Spin Models, Phys. Rev. Letters 92, 015702, (2004).

[G1] J.W. Gibbs, A Method of Geometrical Representation of the Thermodynamic Properties of Substances by Means of Surfaces, Transactions of the Connecticut Academy II, 382-404 (1873). See The Scientific Papers of J. Willard Gibbs, Vol. One, Thermodynamics, 33-54, Ox Bow, Woodbridge, Connecticut (1993).

[G2] J.W. Gibbs, Elementary Principles in Statistical Mechanics, Ox Bow, Woodbridge, Connecticut (1981).

[Ha] P.M. Harman, The Scientific Letters and Papers of James Clerk Maxwell Vol. III 1874-1879, Cambridge University Press (2002).

[HLeb] P.C. Hemmer, J.L. Lebowitz, Systems with Weak Long-Range Potentials in Phase Transitions and Critical Phenomena Vol. 5b, 107-203, Eds. C. Domb, M.S. Green, Academic Press, London (1976).

[vH] L. van Hove, Quelques propriétés générales de l'intégrale de configuration d'un système de particules avec interaction, Physica 15, 951-961 (1949).

[Hu] K. Huang, Statistische Mechanik, Bibliographisches Institut, Mannheim (1964).

[I1] S.N. Isakov, Nonanalytic Features of the First Order Phase Transition in the Ising Model, Commun. Math. Phys. 95, 427443, (1984).

[I2] S.N. Isakov, Phase Diagrams and Singularity at the Point of a Phase Transition of the First Kind in Lattice Gas Models, Teor. Mat. Fiz., 71, 426-440, (1987).

[K] M. Kac, On the partition function of a one-dimensional gas Phys. Fluids 2, 8-12 (1959).

[KUH1] M. Kac, G.E. Uhlenbeck, P.C. Hemmer, On the van der Waals Theory of the Vapor-Liquid Equilibrium I. Discussion of a onedimensional model, J. Math. Phys. 4, 216-228, (1963).

[KUH2] M. Kac, G.E. Uhlenbeck, P.C. Hemmer, On the van der Waals Theory of the Vapor-Liquid Equilibrium II. Discussion of the distribution functions, J. Math. Phys. 4, 229-247, (1963). 
[KUH3] M. Kac, G.E. Uhlenbeck, P.C. Hemmer, On the van der Waals Theory of the Vapor-Liquid Equilibrium III. Discussion of the critical region. J. Math. Phys. 5, 60-74, (1964).

[Ka] B. Kahn, On the theory of the equation of state in Studies in Statistical Mechanics Vol. III, 276-382, Eds. J. De Boer, G.E. Uhlenbeck, North-Holland, Amsterdam (1965).

[KaU] B. Kahn, G.E. Uhlenbeck, On the Theory of Condensation, Physica 5, 399-415 (1938).

[vK] N.G. van Kampen, Condensation of a Classical Gas with LongRange Attraction, Phys. Rev. 135, A362-A369 (1964).

[Kat1] S. Katsura, On the Theory of Condensation, J. Chem. Phys. 22, 1277 (1954).

[Kat2] S. Katsura, Singularities in First-Order Phase Transitions, Adv. Phys. 12, 391-420 (1963).

[Kl] M.J. Klein, The historical origins of the van der Waals equation, Physica 73, 28-47 (1974).

[La1] J.S. Langer, Theory of the Condensation Point, Annals of Physics 41, 108-157 (1967).

[La2] J.S. Langer, Metastable states, Physica 73, 61-72 (1974).

[Leb] J.L. Lebowitz, Exact Derivation of the van der Waals Equation, Physica 73, 48-60 (1974).

[LebP] J.L. Lebowitz, O. Penrose, Rigorous Treatment of the van der Waals-Maxwell Theory of the Liquid-Vapor Transition, J. Math. Phys. 7, 98-113, (1966).

[LeY] T.D. Lee, C.N. Yang, Statistical Theory of State and Phase Transition II, Phys. Rev. 87, 410-419, (1952).

[M1] J.C. Maxwell, Theory of Heat, Dover, New-York (2001).

[M2] J.C. Maxwell, Van der Waals on the Continuity of Gaseous and Liquid States, Nature 10, 477-480 (1874).

[M3] J.C. Maxwell, On the Dynamical Evidence of the Molecular Constitution of Bodies, Nature 11, 357-359, 374-377 (1875); J. Chem. Phys. 13, 493-508 (1875).

[Ma] J.E. Mayer, The Statistical Mechanics of Condensing Systems I J. Chem. Phys 5, 67-73 (1937). 
[MaStr] J.E. Mayer, S.F. Streeter, Phase Transitions J. Chem. Phys. 7, 1019-1025 (1939).

[OV] E. Olivieri, M.E. Vares, Large Deviations and Metastability, Cambridge University Press, Cambridge (2004).

[On] L. Onsager, Crystal statistics. I. A two-dimensional model with an order-disorder transition, Phys. Rev. 65, 117-149 (1944).

[Or] L.S. Ornstein, Application of the statistical mechanics of Gibbs to molecular theoretic questions (in Dutch), Dissertation, Leiden (1908).

[Pe] R. Peierls, On Ising's model of ferromagnetism, Math. Proc. Cambridge Phil. Soc. 32, 477-481 (1936).

[P] O. Penrose, Metastable Decay Rates, Asymptotic Expansions, and Analytic Continuation of Thermodynamic Functions, J. Stat. Phys. 78, 267-283 (1995).

[Pf1] C.-E. Pfister, Large Deviations and Phase Separation in the Two Dimensional Ising Model, Helv. Phys. Acta 64, 953-1054, (1991).

[Pf2] C.-E. Pfister, On the Nature of Isotherms at First Order Phase Transitions. Unpublished lecture notes for the Mark Kac Seminar 2004, available at http://www.win.tue.nl/markkac/ or http://iacs.epfl.ch/ cpfister.

[PiSi] S.A. Pirogov, Y.G. Sinai, Phase Diagrams of Classical Lattice Systems, Teor. Mat. Fiz. 25, 358-369 (1975) and 26, 61-76 (1976).

[Pr] E. Presutti, From Statistical Mechanics to Continuum Mechanics Lecture Notes, Max Planck Institute, Leipzig (1999).

[Rem1] R. Remmert, Theory of Complex Functions, Springer Verlag, Berlin (1991).

[Rem2] R. Remmert, Classical Topics in Complex Analysis, Springer Verlag, Berlin (1991).

[R] J.S. Rowlinson, J.D. van der Waals: On the Continuity of the Gaseous and Liquid States, Edited with an Introductory Essay by J.S. Rowlinson, Studies in Statistical Mechanics Vol. XIV North-Holland, Amsterdam (1988).

[Ru] D. Ruelle, Statistical Mechanics, Benjamin, New York (1969).

[Sh] S.B. Shlosman, Unusual analytic properties of some lattice models: complement of Lee-Yang theory, Teor. Mat. Fiz. 69, 273-278 (1986) 
[Si] Y.G. Sinai, Theory of Phase Transitions: Rigorous Results, Pergamon Press, Oxford (1982).

[St] D.F. Styer, What good is the thermodynamic limit? Am. J. Phys. 72, 25-29 (2004).

[T] H.N.V. Temperley, The Mayer Theory of Condensation Tested Against a Simple Model of Imperfect Gas, Proc. Phys. Soc. 68, 233-238 (1953).

[Th] J. Thomson, Considerations on the Abrupt Change at Boiling or Condensing in Reference to the Continuity of the Fluid State of Matter, Proc. Roy. Soc. 20, 1-8 (1871).

[UF] G.E. Uhlenbeck, G.W. Ford, Lectures in Statistical Mechanics, Lectures in Applied Mathematics Vol. I, A.M.S., reprinted (1986).

[vdW1] J.D. van der Waals, De Continuiteit van den Gas en Vloeistoftoestand, Academic Thesis, Leiden, (1873). English translation in $[\mathrm{R}]$.

[vdW2] J.D. van der Waals, Z. Phys. Chem. 13, 667 (1894). English translation, The Thermodynamic Theory of Capillarity Under the Hypothesis of a Continuous Variation of Density, J. Stat. Phys. 20, 197-244 (1979).

[W1] B. Widom, Surface tension of fluids, in Phase Transitions and Critical Phenomena Vol. 2, 79-100, Eds. C. Domb, M.S. Green, Academic Press, London (1972).

[W2] B. Widom, Structure and thermodynamics of interfaces, in Statistical Mechanics and Statistical Methods in Theory and Application, 33-71 Ed. U. Landsman, Plenum (1977).

[YLe] C.N. Yang, T.D. Lee, Statistical Theory of State and Phase Transition I, Phys. Rev. 87, 404-409, (1952).

[Z] M. Zahradník, An alternate version of Pirogov-Sinai theory, Commun. Math. Phys. 93, 559-581, (1984).

Charles-Edouard Pfister

Ecole Polytechnique Fédérale de Lausanne

EPFL SB IACS, Bât. MA, Station 8

CH-1015 Lausanne, Switzerland

charles.pfister@epfl.ch

http://iacs.epfl.ch/ cpfister 